\title{
$1{ }^{19}$ F NMR-Guided Design of Glycomimetic Langerin 2 Ligands
}

3 Eike-Christian Wamhoff ${ }^{1,2}$, Jonas Hanske ${ }^{1,2}$, Lennart Schnirch ${ }^{2}$, Jonas Aretz ${ }^{1,2}$, Maurice Grube ${ }^{1,2}$,

4 Daniel Varón Silva ${ }^{1,2}$ and Christoph Rademacher ${ }^{1,2} *$

$5 \quad{ }^{1}$ Max Planck Institute of Colloids and Interfaces, Department of Biomolecular Systems, 14424

6 Potsdam, Germany

$7 \quad{ }^{2}$ Freie Universität Berlin, Department of Biology, Chemistry and Pharmacy, 14195 Berlin, Germany

\section{Supporting Information}

9

10 I. Supporting Figures, Tables and Schemes (pp. S2 to S10)

11 II. Methods

- Molecular Modelling (pp. S11 and S12)

- Receptor Expression and Purification (pp. S13 and S14)

- Synthetic Chemistry (pp. S15 to S53)

- ${ }^{19} \mathrm{~F} \mathrm{R}_{2}$-filtered NMR (pp. S54 to S55)

- ${ }^{15}$ N HSQC NMR (p. S56)

- $\quad{ }^{1}$ H STD NMR (p. S57)

III. References (pp. S58 and S59) 


\section{Supporting Figures, Tables and Schemes}

20 Scheme S1. Synthesis of 2-carboxamido-2-deoxy- $\alpha$-mannoside and $\boldsymbol{\alpha}$-mannoside analogs 5. a. Reaction conditions for the preparation of precursor 4: (a) acetone: $\mathrm{H}_{2} \mathrm{O}(1: 1), 50^{\circ} \mathrm{C}$; (b) pyridine, $50^{\circ} \mathrm{C}$; (c) $\mathrm{BF}_{3} \cdot \mathrm{OEt}_{2}$, anhydrous DCM:ether (2:1), $0^{\circ} \mathrm{C}$ to room temperature; (d) $\mathrm{EtOH}$, room temperature. b. Reaction conditions for the preparation of 2-carboxamido-2deoxy- $\alpha$-mannoside analogs 5: (e) $\mathrm{MeOH}$, room temperature; (f) DMF, room temperature; (g) pyridine, room temperature; (h) PyBOP, DIPEA, DMF, room temperature. c. Reaction conditions for the preparation of $\alpha$-mannoside analog 5.0.

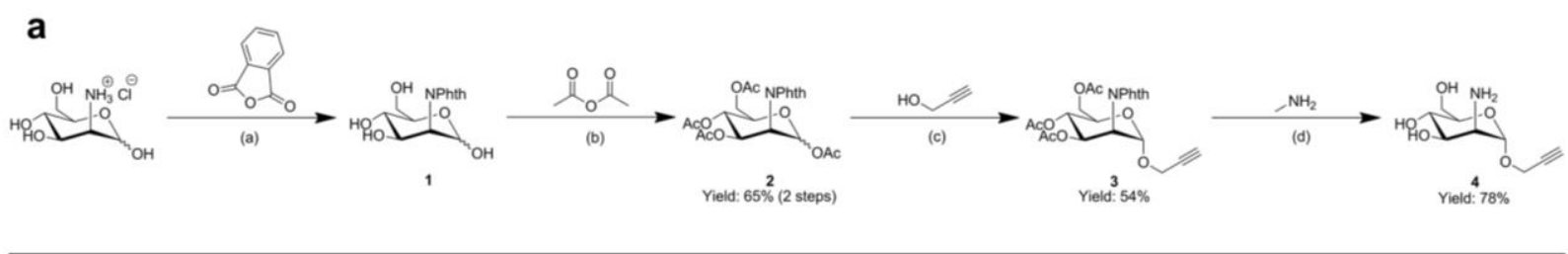

b

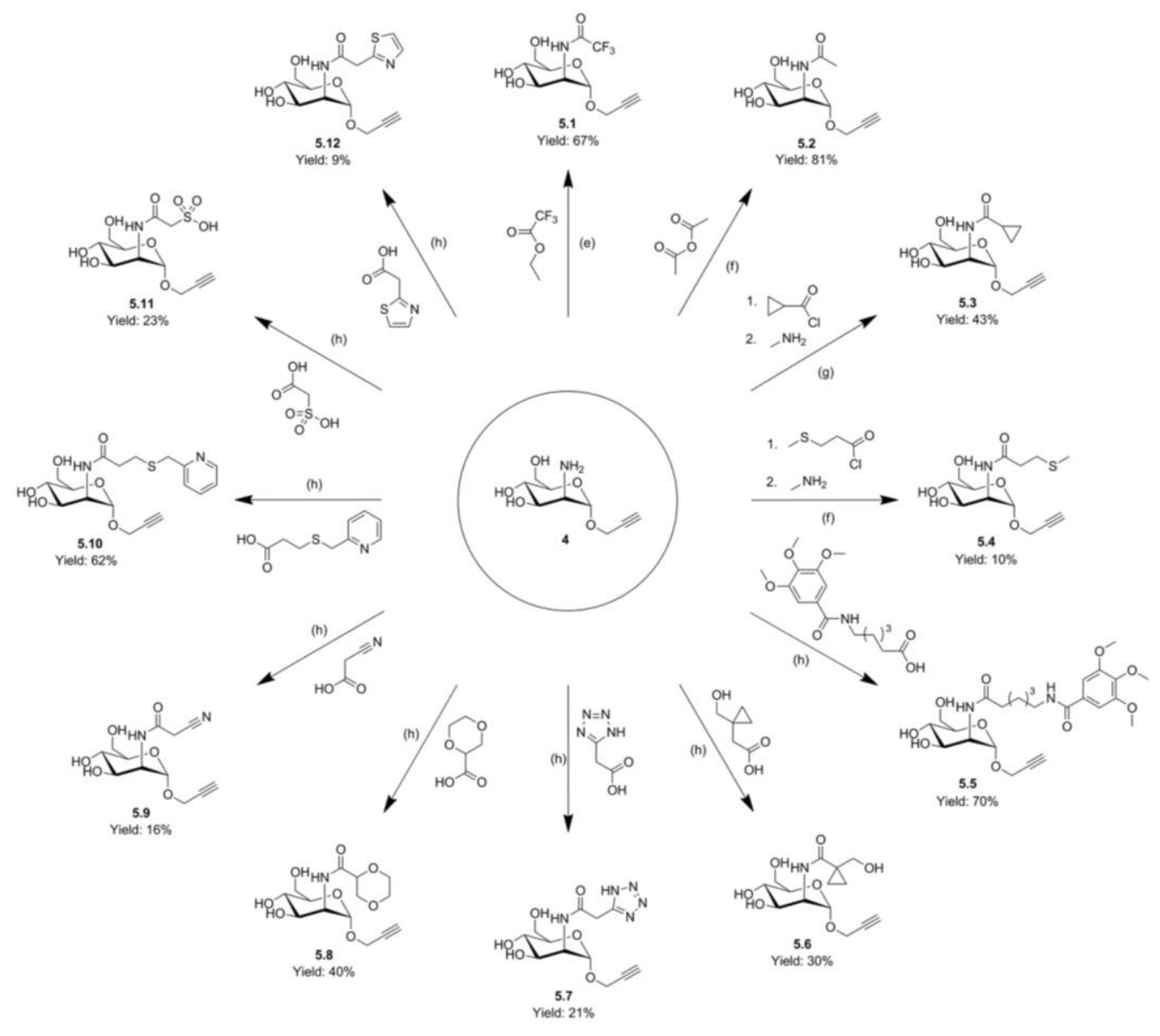

C

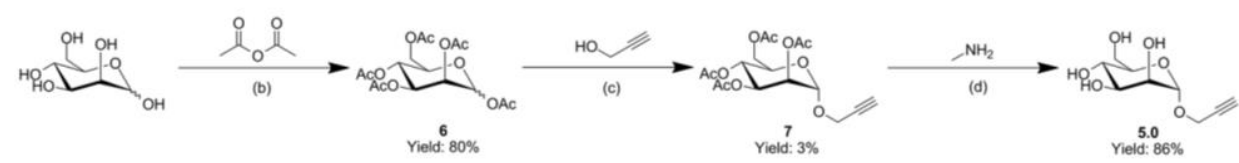


a

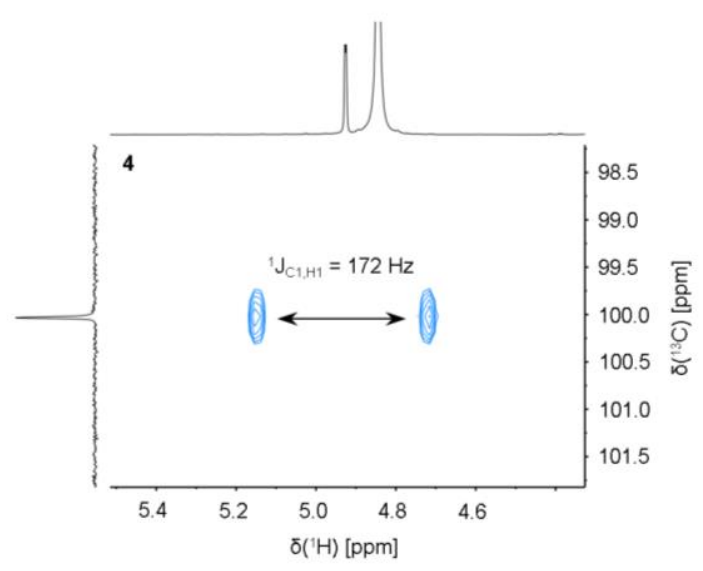

C

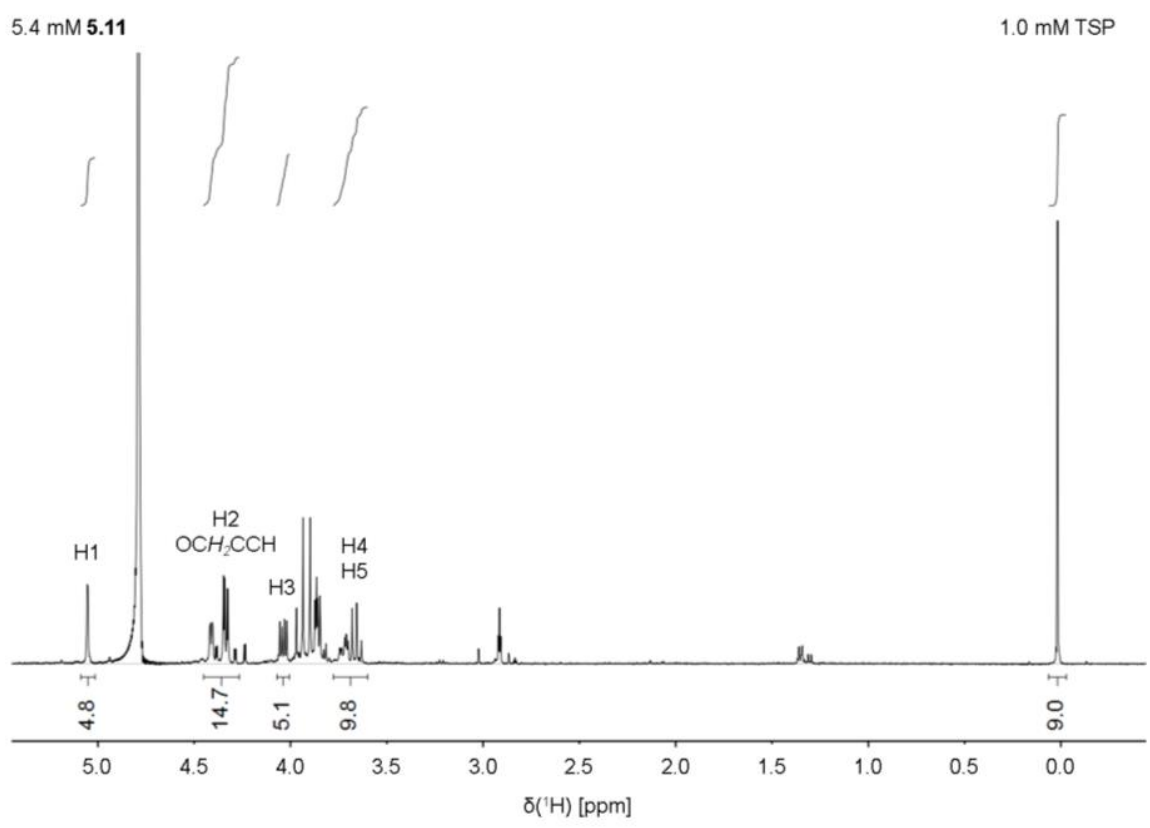

b

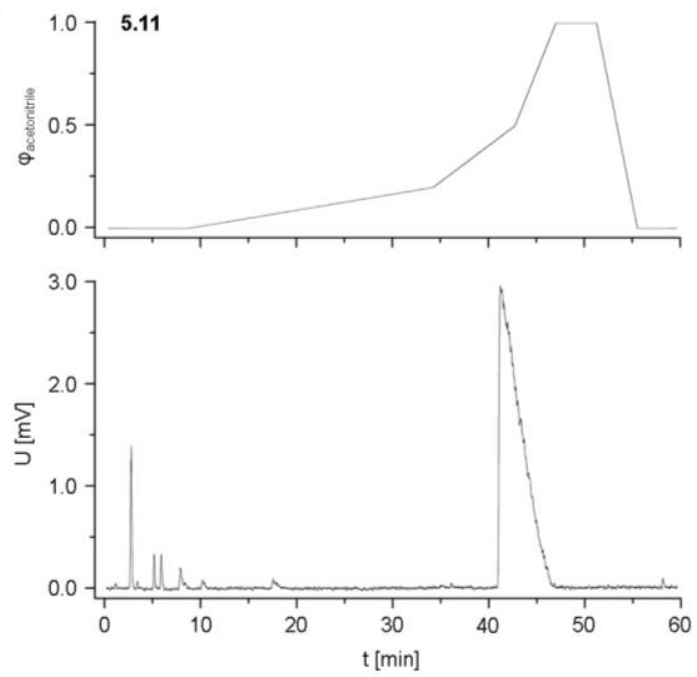

Figure S1. Supporting information for the synthesis of 2-carboxamido-2-deoxy- $\alpha$-mannoside analogs 5. a. The $\alpha$ configuration of precursor 4 was validated via the determination of the coupling constant ${ }^{1} \mathrm{~J}_{\mathrm{C} 1, \mathrm{H} 1}$ from ${ }^{13} \mathrm{C}$ HSQC NMR spectra (1). b. The purity of $\mathbf{5 . 1 1}$ was analyzed via analytical HPLC run on a HyperCarb column using a $0.1 \%$ FA in $\mathrm{H}_{2} \mathrm{O}-$ acetonitrile gradient, a flow rate of $1.0 \mathrm{ml} \cdot \mathrm{min}^{-1}$ and ELSD. c. ${ }^{1} \mathrm{H}$ NMR experiments in presence of TSP in $\mathrm{D}_{2} \mathrm{O}$ revealed a purity of $89 \%$ to $94 \%$ for $\mathbf{5 . 1 1}$, depending on the integrated resonances. 
a

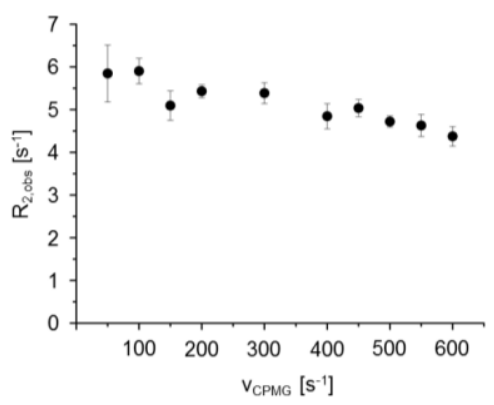

d

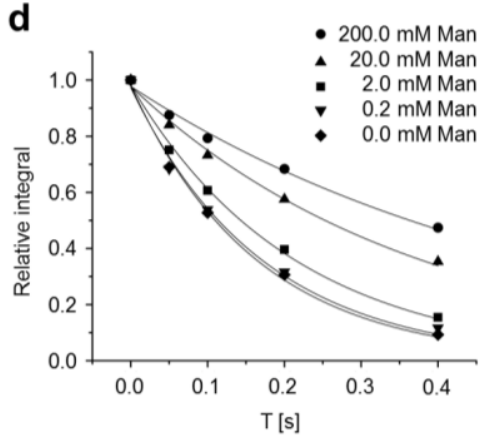

b

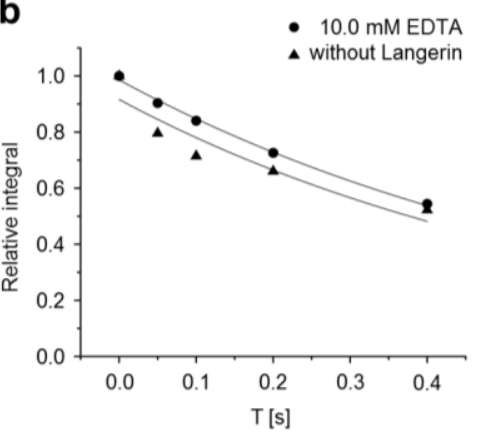

e

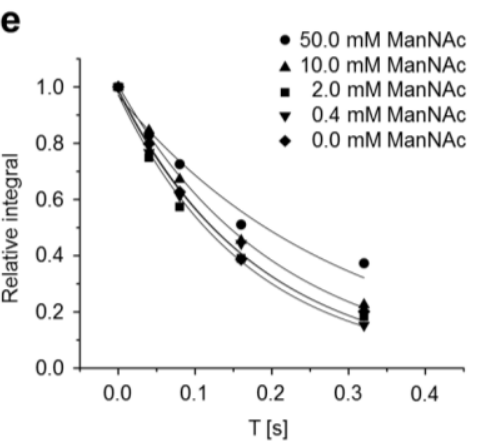

C

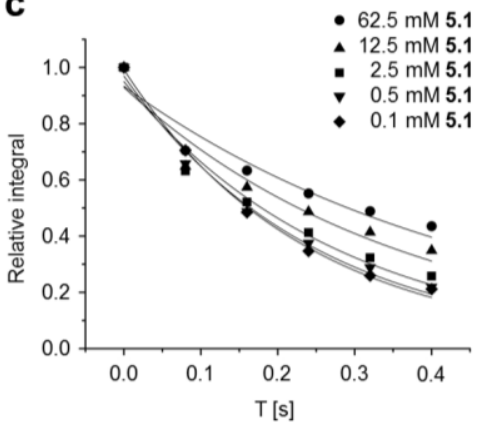

f

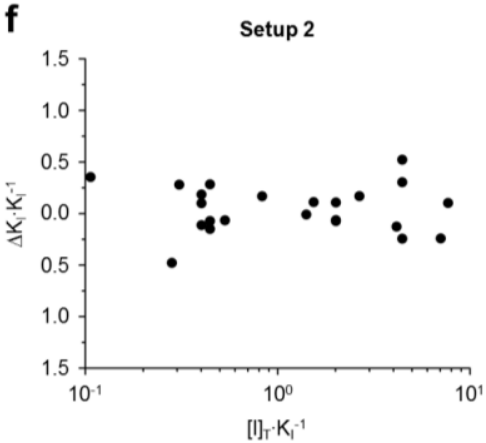

Figure S2. Supporting information for the ${ }^{19} \mathbf{F} \mathbf{R}_{2}$-filtered NMR assay. a. Relaxation dispersion experiments at $0.1 \mathrm{mM}$ 5.1 in presence of the Langerin ECD indicate a negligible exchange contribution $R_{2, e x}$ at a $v_{\text {CPMG }}$ value of $500 \mathrm{~Hz}$. b. The decay curve at $12.5 \mathrm{mM} \mathrm{5.1}$ in presence of the ECD and EDTA is depicted. The comparison with a representative decay curve at $0.1 \mathrm{mM} 5.1$ in absence of Langerin validates the $\mathrm{Ca}^{2+}$-dependency of the interaction. $\mathbf{c}$. The decay curves for the titration with 5.1 in presence of the CRD are shown. d. and e. Representative decay curves from the competitive binding experiments with Man and ManNAc are shown. f. 23 data points selected from competitive binding experiments with 5.7, 5.8, 5.9, 5.11, Man and ManNAc served to simulate the assay performance in Setup 2.

Table S1. Supporting information for the ${ }^{19} \mathbf{F} \mathbf{R}_{2}$-filtered NMR assay.

$$
\text { ECD CRD }
$$

$\begin{array}{lrr} & & \\ \mathrm{R}_{2, \mathrm{f}}\left[\mathrm{s}^{-1}\right] & 1.8 \pm 0.2^{\mathrm{a}} & 1.8 \pm 0.2^{\mathrm{a}} \\ \mathrm{R}_{2, \mathrm{~b}}\left[\mathrm{~s}^{-1}\right] & 660.3 \pm 48.2^{\mathrm{b}} & 361.5 \pm 46.5 \\ \mathrm{~K}_{\mathrm{D}}[\mathrm{mM}] & 7.9 \pm 0.7^{\mathrm{b}} & 7.3 \pm 1.0\end{array}$


a

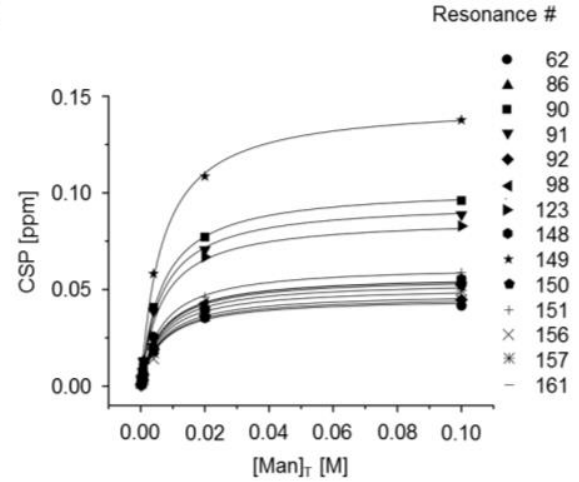

C

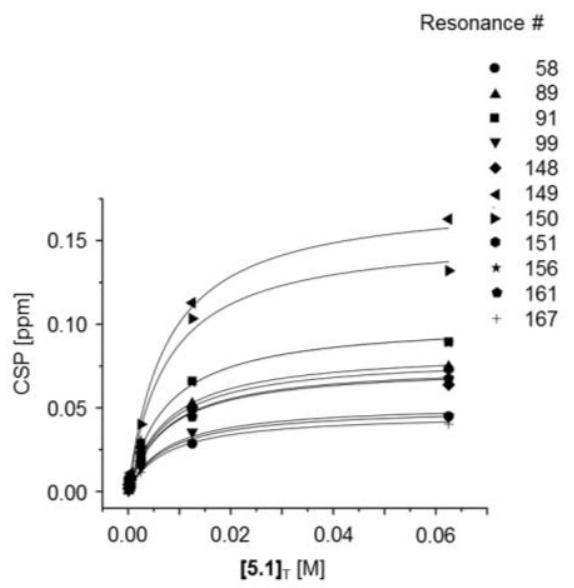

b

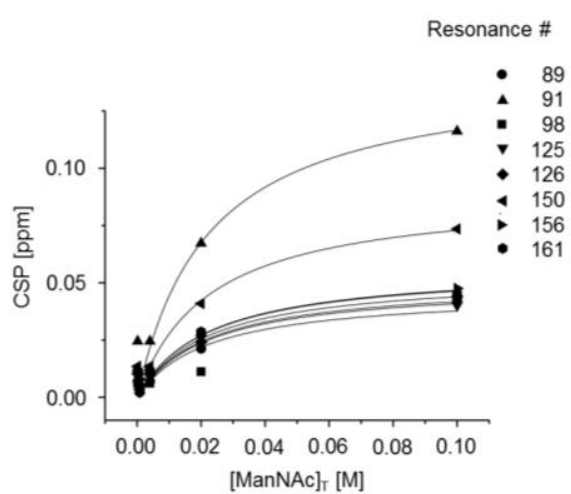

d

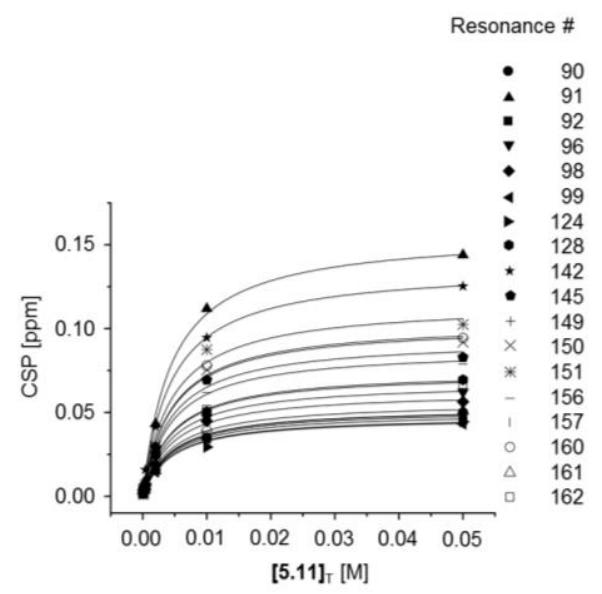

e

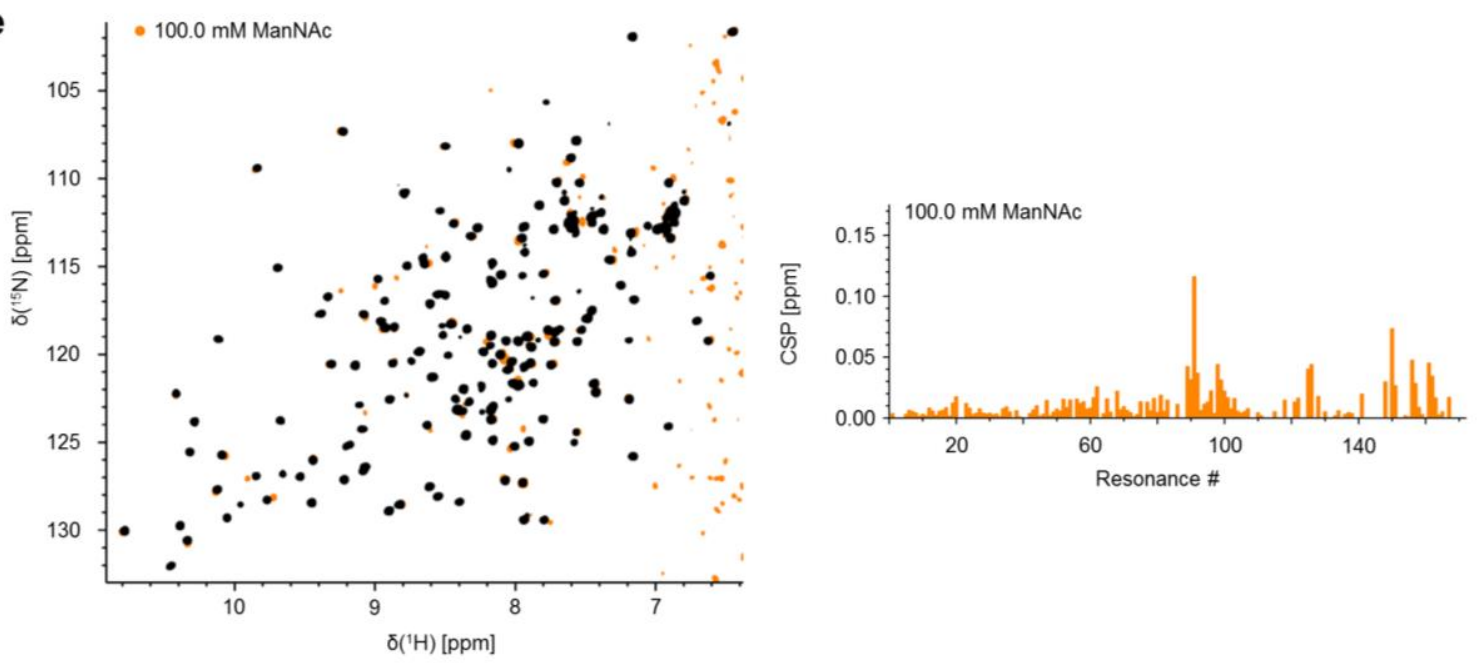

Figure S3. Supporting information for the ${ }^{15}$ N HSQC NMR assay. a. to $d$. Resonances with CSP values higher than 0.04 $\mathrm{ppm}$ at the highest ligand concentration were selected for the determination of the $K_{\mathrm{D}}$ values of Man, ManNAc, 5.1 and 5.11. e. The CSP fingerprint observed for ManNAc suggests a binding mode similar to that of Man, 5.1 and 5.11. 
51 Table S2. Comparison of CSP fingerprints for Man, 5.1 and 5.11. For all resonances that display a CSP higher than 0.04 $52 \mathrm{ppm}$ in presence of $100.0 \mathrm{mM}$ Man, CSPs are also observed upon titration with $62.5 \mathrm{mM} 5.1$ or $50.0 \mathrm{mM} \mathrm{5.11}$. These 53 findings suggest a similar binding mode.

\begin{tabular}{lrrr} 
& \multicolumn{3}{c}{ CSP [ppm] } \\
Resonance \# & $100.0 \mathrm{mM}$ Man & $62.5 \mathrm{mM} \mathbf{5 . 1}$ & $50.0 \mathrm{mM} \mathbf{5 . 1 1}$ \\
& & & \\
\hline & & & \\
62 & 0.041 & 0.029 & 0.039 \\
86 & 0.055 & 0.025 & 0.027 \\
90 & 0.096 & 0.022 & 0.050 \\
91 & 0.089 & 0.090 & 0.144 \\
92 & 0.045 & 0.023 & 0.045 \\
98 & 0.052 & 0.020 & 0.056 \\
123 & 0.083 & n.d.* & 0.020 \\
148 & 0.055 & 0.064 & 0.050 \\
149 & 0.138 & 0.163 & 0.139 \\
150 & 0.053 & 0.132 & 0.092 \\
151 & 0.059 & 0.073 & 0.102 \\
156 & 0.044 & 0.089 & 0.079 \\
157 & 0.050 & 0.010 & 0.049 \\
161 & 0.054 & 0.068 & 0.051
\end{tabular}





Figure S4. Structure-based in silico design of 2-carboxamido-2-deoxy- $\alpha$-mannoside analogs 5. a. Man recognition by Langerin is driven by the $\mathrm{Ca}^{2+}$-coordination via two equatorial hydroxyl groups. Only few secondary interactions are observed (2). b. The surface representation reveals two pockets in axial direction of C2 of the Man scaffold. Hydrophilic regions of the receptor surface are depicted in blue while hydrophobic regions are depicted in red. c. A structural alignment of the binding sites of available X-ray structures of Langerin in complex with different oligomannosides is depicted (3P5D.pdb, 3P5E.pdb and 3P5F.pdb) (2). The orientation of the directly bound Man is highly conserved. d. A pharmacophore model was defined to constrain the orientation of the Man scaffold during the force field-based refinement of generated docking poses. All features displayed require an oxygen atom within the indicated spheres. e. and f. Different amide linker conformations were selected from low mode molecular dynamics simulations and utilized for the in situ conjugation of commercially available carboxylic acids. Moreover, the alternative conformation of K313 observed for the complex with Gal-6S was accounted for in additional docking runs (2). g. The distribution of hits with respect to the predicted affinity increase $\mathrm{A}$ and the corresponding group efficiency $\mathrm{GE}_{\text {pred }}$ is depicted. The focused library of analogs $\mathbf{5}$ was selected from hits displaying a $\mathrm{GE}_{\text {pred }}$ value higher than $0.15 \mathrm{~kJ} \cdot \mathrm{mol}^{-1}$. 


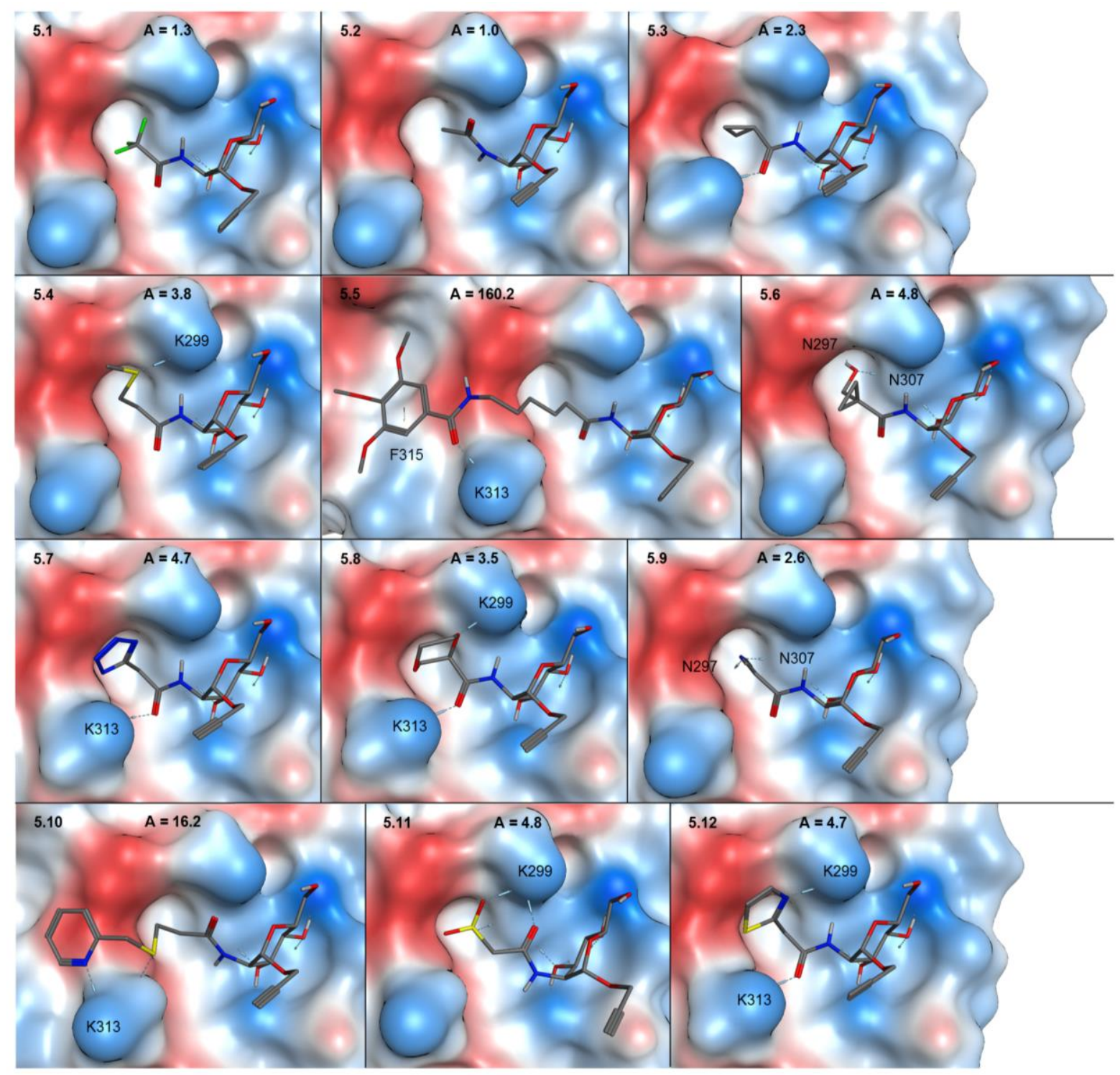

Figure S5. Docking poses for 2-carboxamido-2-deoxy-a-mannoside analogs 5. For each analog 5 the docking pose with the highest GBVI/WSA $\Delta \mathrm{G}$ score is depicted. The corresponding scores served to predict the affinity increase over 5.2. Residues involved in directed interactions with the substituents in $\mathrm{C} 2$ of the Man scaffold are indicated. Hydrophilic regions of the receptor surface are depicted in blue while hydrophobic regions are depicted in red. 

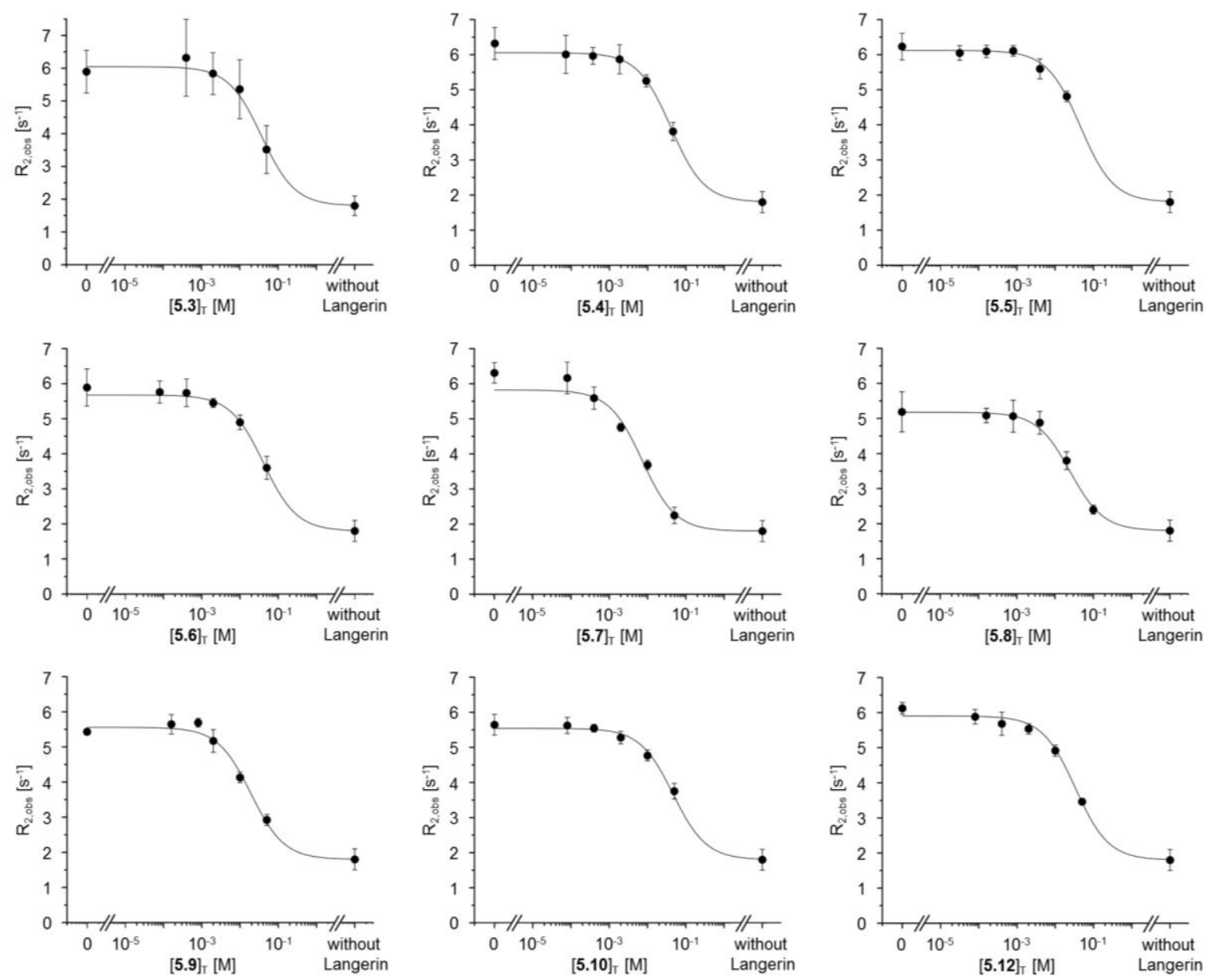

Figure S6. Determination of $K_{I}$ values for 2-carboxamido-2-deoxy- $\alpha$-mannoside analogs 5. Equation 3 was fitted to recorded $\mathrm{R}_{2, \text { obs }}$ values to determine $[\mathrm{P}]_{\mathrm{T}}$ and $\mathrm{K}_{\mathrm{I}}$ values. The results are summarized in Table 1 . 

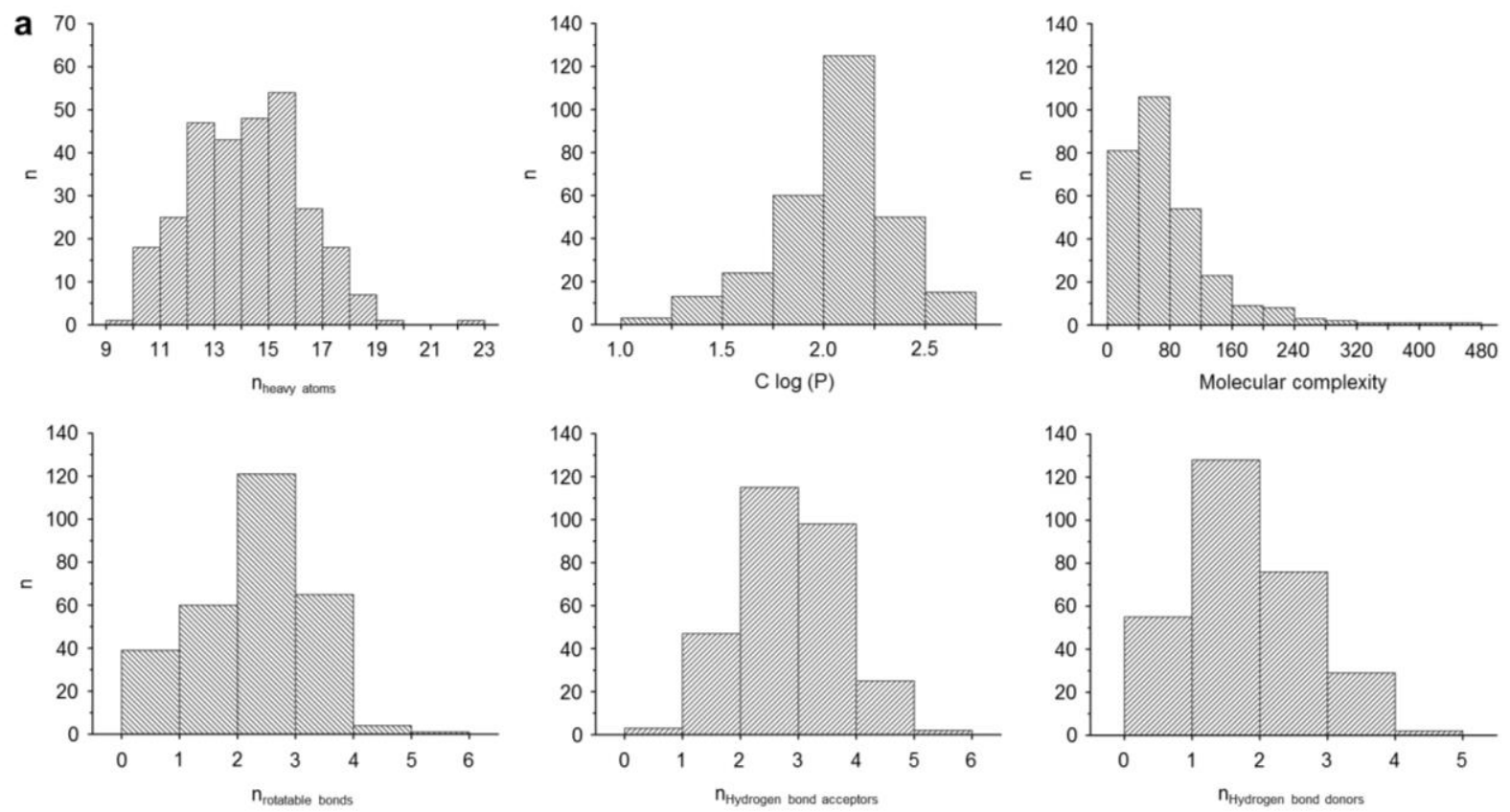

b

C
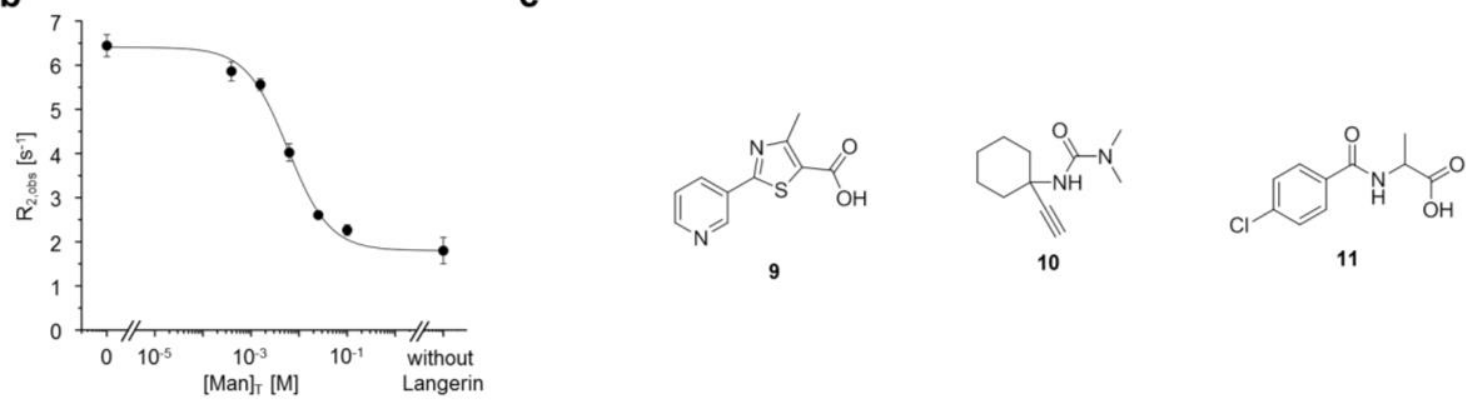

d

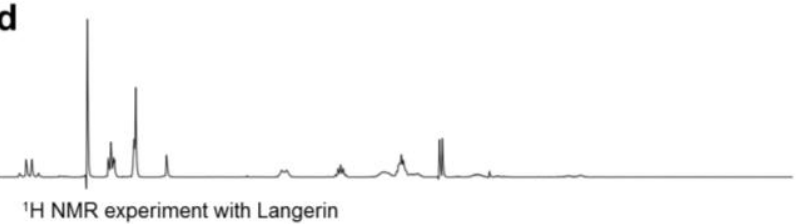

e

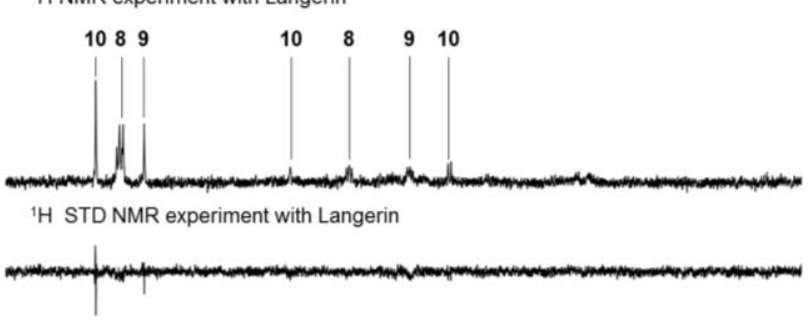

1H STD NMR experiment without Langerin
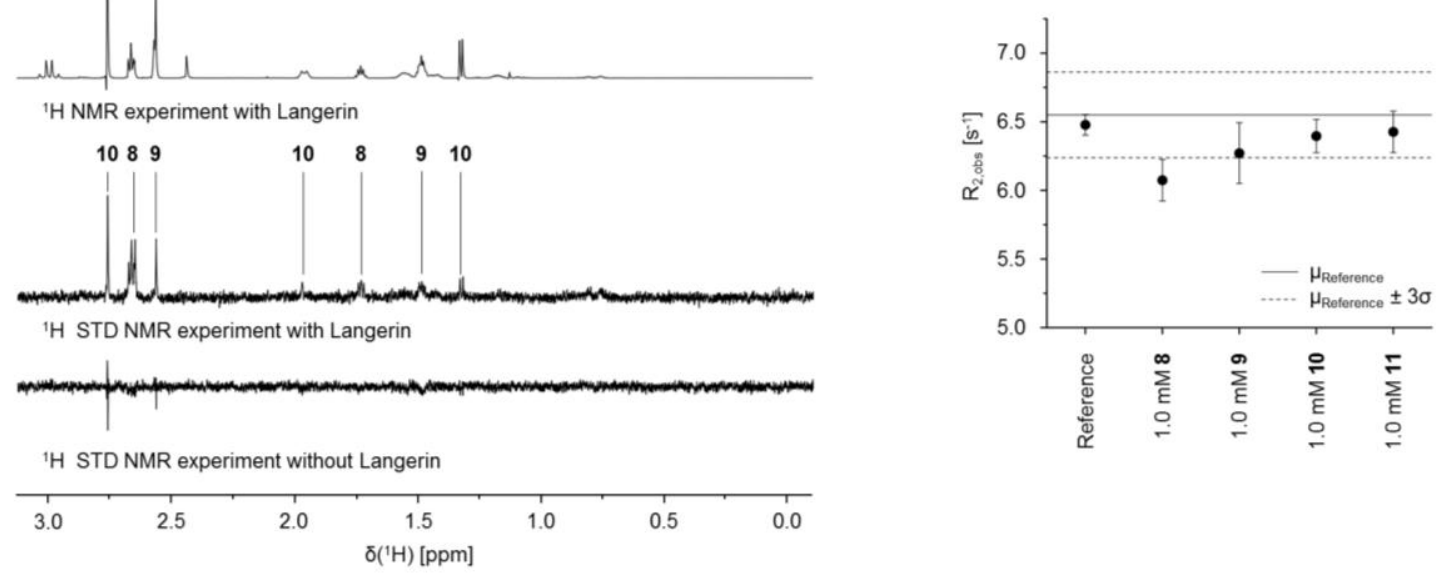

Figure S7. Supporting information for the explorative fragment screening. a. 290 fragments were randomly selected from our in-house fragment library and screened against Langerin with the ${ }^{19} \mathrm{~F} \mathrm{R}_{2}$-filtered NMR assay. Relevant descriptors such as $\mathrm{C} \log (\mathrm{P})$, molecular complexity as well as the number of heavy atoms, non-terminal rotatable bonds, hydrogen bond acceptors and hydrogen bond donors are compliant with published guidelines for fragment library design (3-5). b. Fragment mixtures were screened in presence of $10 \%$ DMSO and $0.01 \%$ Tween-20. The determined $\mathrm{K}_{\mathrm{I}}$ value of $5.6 \pm 0.2 \mathrm{mM}$ for Man in presence of these additives is comparable to the affinity obtained in their absence. c. and d. The fragment mixture displaying the highest inhibition was analyzed by ${ }^{1} \mathrm{H}$ STD NMR. Fragments 8, 9, $\mathbf{1 0}$ and $\mathbf{1 1}$ were found to interact with Langerin. STD spectra are magnified 25 -fold. e. Upon deconvolution of this mixture, only fragment $\mathbf{8}$ was observed to compete with reporter molecule 5.1. 


\section{Methods}

\section{Molecular Modelling}

\section{General remarks}

Molecular modelling procedures were performed in MOE (6). Unless stated otherwise, options and parameters were set to default. The AMBER10:EHT force field was selected for the refinement of docking poses and the hydrogen bond network while MMFF94x was utilized for the generation of carboxylic acid conformers (7-9). Databases were processed in KNIME and tautomers were enumerated with ChemAxon's Calculator Plugin (10).

\section{Definition of the pharmacophore model and preparation of the Langerin complex}

A structural alignment of Langerin carbohydrate binding sites of in complex with different oligomannosides was performed (3P5D.pdb, 3P5E.pdb and 3P5F.pdb) (2). Based on this visualization, a pharmacophore model was defined with features for O3, O4 and O5 of the Man scaffold. The spatial constraint on the $\mathrm{O} 3$ and $\mathrm{O} 4$ was defined by a sphere with a radius $\mathrm{r}$ of $0.5 \AA$ while the position of $\mathrm{O} 5$ was constrained by a sphere with a radius $r$ of $1.0 \AA$. Chain $B$ of the Langerin CRD in complex with a dimannoside served as the structural basis for the performed in silico screening (3P5F.pdb). Of the two binding modes included in this model, the orientation for targeting the identified pockets in axial direction of C2 was selected. Additionally, an alternative conformation for K313 observed for the Langerin complex with Gal-6S was modeled and included into the analysis (11). Overall model quality and protein geometry were evaluated in MolProbity (12). Next, protonation states and the hydrogen bond network of the complex were simulated with MOE's Protonate 3D followed by the removal of all solvent molecules. Subsequently, a propargyl group was modeled to the anomeric position of the mannose scaffold and the axial hydroxyl group in C2 was substituted with an acetamido group. The conformational space for the dihedral angle of the $\mathrm{C} 2-\mathrm{N}$ bond was explored in context of the binding site via Low Mode MD simulations assuming trans configuration of the acetamido group (13). Five energetically favorable rotamers corresponding to two different conformations of K313 were identified and served as the structural basis for the subsequent in silico screening of the carboxylic acid conformation database against Langerin.

\section{Preparation of the carboxylic acid conformation database}

Carboxylic acid conformations were generated from building block databases of selected manufacturers (TimTec, TCI, Sigma Aldrich, Otava Chemicals, Life Chemicals, Focus Synthesis, Enamine, ChemDiv, ChemBridge and Asinex). The database was processed with MOE's Wash and 
121 filtered to yield structures with a maximum of 23 heavy atoms and 6 rotatable bonds. Moreover, only

122 molecules containing one carboxyl group and no amino, azido or alkyne group were retained. Next,

123 this subset was filtered for reactive molecules followed by the generation of tautomers and protonation

124 states with ChemAxon's Calculator Plugin $(14,15)$. Subsequently, the carboxyl group was removed

125 and substituted by an annotated atom with MOE's Combinatorial Library that served as an annotation

126 point for virtual conjugation to the modified Man scaffold in situ.

\section{In silico screening of the conformation database against Langerin}

128 The docking procedure was implemented with MOE's Combinatorial Builder. The carboxylic acids 129 were conjugated to the modified Man scaffold by substituting the terminal methyl group via the virtual

130 formation of a C-C bond. A grid-based placement method was utilized for generating docking poses

131 by exploring the conformational space around this bond. During the subsequent force field-based

132 structure refinement, the binding mode of the mannose scaffold was constrained by the 133 pharmacophore model described above. Conformational flexibility of the binding site was accounted 134 for by introducing B-factor-derived tethers to side chain atoms. Refined docking poses were then 135 filtered by the pharmacophore model, scored with the GBIV/WSA $\Delta \mathrm{G}$ function and written into the 136 output database. Next, scores were referenced against $\mathbf{5 . 2}$ and calculated GBIV/WSA $\Delta \Delta \mathrm{G}$ values 137 served to determine the predicted group efficiency $\mathrm{GE}_{\text {pred }}$ as well as the predicted affinities increase $\mathrm{A}$. 138 Only poses with a $\mathrm{GE}_{\text {pred }}$ value higher than $0.15 \mathrm{~kJ} \cdot \mathrm{mol}^{-1}$ and an RMSD upon refinement lower than 2 $139 \AA$ were retained. Highly scored 2-deoxy-2-carboxamido- $\alpha$-mannoside analogs 5 were evaluated 140 visually and a focused library was composed. The composition of the library was guided by an attempt 141 to maximize the diversity of pharmacophore features and to ensure synthetic feasibility. Importantly, 142 this first generation of analogs was selected to test basic binding hypotheses and to establish a 143 structure activity relationship in axial direction of the $\mathrm{C} 2$. 


\section{General remarks}

Codon-optimized genes for the expression of Langerin in E. coli were purchased from GenScript. All growth media or chemicals used for receptor expression and purification were purchased from Carl Roth if not stated otherwise.

\section{Langerin ECD}

The truncated Langerin ECD (residues 148 to 328, forward primer: GGTGGTCATATGGCCTCGAC GCTGAATGCCCAGATTCCGG, reverse primer: ACCACCAAGCTTTTATTTTTCAAACTGCGG ATG) was cloned with a C-terminal TEV cleavage site and a Strep-tag II into a pET30a expression vector (EMD Millipore) and expressed insolubly in E. coli BL21 ${ }^{*}$ (DE3) (Invitrogen). Precultures were incubated overnight in LB medium supplemented with $35 \mu \mathrm{g} \cdot \mathrm{ml}^{-1}$ Kanamycin $(50 \mathrm{ml})$ at $37^{\circ} \mathrm{C}$ and $220 \mathrm{rpm}$. The preculture was diluted to an $\mathrm{OD}_{600}$ of 0.1 into LB medium supplemented with 35 $\mathrm{mg} \cdot \mathrm{ml}^{-1}$ Kanamycin $(500 \mathrm{ml})$. The culture was incubated at $37^{\circ} \mathrm{C}$ and $220 \mathrm{rpm}$ and expression of the Langerin ECD was induced with $0.5 \mathrm{mM}$ IPTG at an $\mathrm{OD}_{600}$ of 0.6 to 0.8 . Cells were harvested $4 \mathrm{~h}$ after induction via centrifugation at $4000 \mathrm{~g}$ and $4^{\circ} \mathrm{C}$ for $20 \mathrm{~min}$. Cell pellets were stored overnight at $20^{\circ} \mathrm{C}$ and subsequently resuspended in $50 \mathrm{mM}$ Tris with $0.1 \%$ Triton $\mathrm{X}-100$ and $10 \mathrm{mM} \mathrm{MgCl}_{2}(20$ $\mathrm{ml}$ ) at $\mathrm{pH}$ 7.5. Lysozyme (Sigma Aldrich) was added and the sample was incubated for $3.5 \mathrm{~h}$ at $4^{\circ} \mathrm{C}$. After the addition of DNase I (AppliChem) the sample was incubated for another $30 \mathrm{~min}$ at $4^{\circ} \mathrm{C}$. Inclusion bodies were harvested via centrifugation at $10000 \mathrm{~g}$ and $4^{\circ} \mathrm{C}$ for $10 \mathrm{~min}$ and washed three times with $25 \mathrm{mM}$ Tris with $150 \mathrm{mM} \mathrm{NaCl}(20 \mathrm{ml})$ at $\mathrm{pH}$ 7.8. Inclusion body pellets were stored overnight at $-20^{\circ} \mathrm{C}$ and subsequently solubilized overnight in $100 \mathrm{mM}$ Tris with $6 \mathrm{M} \mathrm{Gu}-\mathrm{HCl}$ and 1 $\mathrm{mM}$ DTT $(20 \mathrm{ml})$ at $\mathrm{pH} 8.0$ and $30^{\circ} \mathrm{C}$. Following centrifugation at $15000 \mathrm{~g}$ and $4^{\circ} \mathrm{C}$ for $1.5 \mathrm{~h}$, the Langerin ECD was refolded overnight via rapid dilution into $50 \mathrm{mM}$ Tris with $0.4 \mathrm{M}$ arginine, $20 \mathrm{mM}$ $\mathrm{NaCl}, 0.8 \mathrm{mM} \mathrm{KCl}, 1 \mathrm{mM}$ glutathione (AppliChem) and $0.2 \mathrm{mM}$ glutathione disulfide (AppliChem) $(200 \mathrm{ml})$ at $\mathrm{pH} 7.6$ and $4^{\circ} \mathrm{C}$. Next, the sample was dialyzed overnight against $25 \mathrm{mM}$ Tris, $150 \mathrm{mM}$ $\mathrm{NaCl}, 25 \mathrm{mM} \mathrm{CaCl}_{2}$ at $\mathrm{pH} 7.8$ and $4^{\circ} \mathrm{C}$. After centrifugation at $15000 \mathrm{~g}$ and $4^{\circ} \mathrm{C}$ for $2 \mathrm{~h}$, the sample was purified as via mannan-agarose (Sigma Aldrich, St. Louis, USA) affinity chromatography as previously published (16). The buffer was exchanged to $25 \mathrm{mM}$ Tris with $150 \mathrm{mM} \mathrm{NaCl}$ at pH 7.8 via $7 \mathrm{kDa}$ size-exclusion desalting columns (Thermo Scientific) and the concentration of Langerin ECD was determined via UV spectroscopy $\left(\mathrm{A}_{280,0.1 \%}=2.45\right)(17)$. Typical yields were in the range are of 10 $\mathrm{mg} \cdot \mathrm{l}^{-1}$ bacterial culture. Purity and monodispersity of Langerin ECD samples were analyzed via SDS PAGE and DLS, respectively. 


\section{Langerin CRD}

177

178

179

180

181

182

183

184

185

186

187

188

189

190

191

192

193

194

195

196

197

198

199

200

201

202

203

204

The Langerin CRD (residues 193 to 328, forward primer: GGTGGTCATATGGCCCAGGTGGTTAG CCAAGGCTGGAAATAC, reverse primer: ACCACCAAGCTTTTATTTTTCAAACTGCGGATG) was cloned with a C-terminal TEV cleavage site and a Strep-tag II into a pET30a expression vector (Invitrogen) and expressed insolubly in E. coli BL21* (DE3) (Invitrogen). Precultures were incubated overnight in M9 medium supplemented with $35 \mu \mathrm{g} \cdot \mathrm{ml}^{-1}$ Kanamycin and ${ }^{15} \mathrm{~N}$-labeled $\mathrm{NH}_{4} \mathrm{Cl}$ (Sigma Aldrich) $(50 \mathrm{ml})$ at $37^{\circ} \mathrm{C}$ and $220 \mathrm{rpm}$. The preculture was diluted to an $\mathrm{OD}_{600}$ of 0.1 into $\mathrm{M} 9$ medium supplemented with $35 \mathrm{mg} \cdot \mathrm{ml}^{-1}$ Kanamycin and ${ }^{15} \mathrm{~N}$-labeled $\mathrm{NH}_{4} \mathrm{Cl}$ (Sigma Aldrich) $(500 \mathrm{ml})$. The culture was incubated at $37^{\circ} \mathrm{C}$ and $220 \mathrm{rpm}$ and expression of the Langerin CRD was induced with $0.5 \mathrm{mM}$ IPTG at an $\mathrm{OD}_{600}$ of 0.6 to 0.8 . Cells were harvested $4 \mathrm{~h}$ after induction via centrifugation at $4000 \mathrm{~g}$ and $4^{\circ} \mathrm{C}$ for $20 \mathrm{~min}$. Cell pellets were stored overnight at $-20^{\circ} \mathrm{C}$ and subsequently resuspended in $50 \mathrm{mM}$ Tris with $0.1 \%$ Triton $\mathrm{X}-100$ and $10 \mathrm{mM} \mathrm{MgCl}_{2}(20 \mathrm{ml})$ at $\mathrm{pH}$ 7.5. Lysozyme (Sigma Aldrich) was added and the sample was incubated for $3.5 \mathrm{~h}$ at $4^{\circ} \mathrm{C}$. After the addition of DNase I (AppliChem) the sample was incubated for another $30 \mathrm{~min}$ at $4^{\circ} \mathrm{C}$. Inclusion bodies were harvested via centrifugation at $10000 \mathrm{~g}$ and $4^{\circ} \mathrm{C}$ for $10 \mathrm{~min}$ and washed three with $25 \mathrm{mM}$ Tris with $150 \mathrm{mM}$ $\mathrm{NaCl}(20 \mathrm{ml})$ at $\mathrm{pH}$ 7.8. Inclusion body pellets were stored overnight at $-20^{\circ} \mathrm{C}$ and subsequently solubilized overnight in $100 \mathrm{mM}$ Tris with $6 \mathrm{M} \mathrm{Gu}-\mathrm{HCl}$ and $1 \mathrm{mM}$ DTT $(20 \mathrm{ml})$ at $\mathrm{pH} 8.0$ and $30^{\circ} \mathrm{C}$. Following centrifugation at $15000 \mathrm{~g}$ and $4^{\circ} \mathrm{C}$ for $1.5 \mathrm{~h}$, the Langerin CRD was refolded overnight via rapid dilution into $50 \mathrm{mM}$ Tris with $0.8 \mathrm{M}$ arginine, $20 \mathrm{mM} \mathrm{NaCl}, 0.8 \mathrm{mM} \mathrm{KCl}, 1 \mathrm{mM}$ glutathione (AppliChem) and $0.2 \mathrm{mM}$ glutathione disulfide (AppliChem) $(200 \mathrm{ml})$ at $\mathrm{pH} 7.6$ and $4^{\circ} \mathrm{C}$. Next, the sample was dialyzed overnight against $50 \mathrm{mM}$ Tris, $150 \mathrm{mM} \mathrm{NaCl}, 1 \mathrm{mM}$ EDTA at $\mathrm{pH} 8.0$ and $4^{\circ} \mathrm{C}$. After centrifugation at $15000 \mathrm{~g}$ and $4^{\circ} \mathrm{C}$ for $2 \mathrm{~h}$, the sample was purified as via StrepTactin affinity chromatography (Iba). The Langerin CRD was eluted with $50 \mathrm{mM}$ Tris with $2.5 \mathrm{mM}$ d-desthiobiotin, $150 \mathrm{mM} \mathrm{NaCl}$ at $\mathrm{pH} 7.5$ and dialyzed against $25 \mathrm{mM}$ MES with $40 \mathrm{mM} \mathrm{NaCl}$ at $\mathrm{pH}$ 6.0. After centrifugation at $15000 \mathrm{~g}$ and $4^{\circ} \mathrm{C}$ for $1.5 \mathrm{~h}$, the buffer was exchanged to $25 \mathrm{mM}$ HEPES with 150 $\mathrm{mM} \mathrm{NaCl}$ at $\mathrm{pH} 7.0$ via $7 \mathrm{kDa}$ size-exclusion desalting columns (Thermo Scientific) and the concentration of Langerin CRD was determined via UV spectroscopy $\left(A_{280,0.1 \%}=3.19\right)$ (17). Typical yields were in the range are of $5 \mathrm{mg} \cdot \mathrm{l}^{-1}$ bacterial culture. Purity and monodispersity of Langerin CRD samples were analyzed via SDS PAGE and DLS, respectively. 


\section{Synthetic Chemistry}

\section{General remarks}

Reagents and solvents used were purchased from Sigma Aldrich unless indicated otherwise and used as supplied without any further purification. Anhydrous solvents were taken from an anhydrous solvent system (JC-Meyer Solvent Systems). Column chromatography was carried out using silica gel at a pore size from 40 to $60 \AA$ (Machery Nagel). Reversed-phase column chromatography was carried out using Chromabond endcapped $\mathrm{C}_{18}$ columns at a pore size of $60 \AA$ (Machery Nagel). Analytical TLC was performed on glass plates coated with silica gel at a pore size of $60 \AA$ (Machery Nagel). Compounds were detected via 3-methoxyphenol reagent (0.2\% 3-methoxyphenol in EtOH: $2 \mathrm{~N}$ sulfuric acid in $\mathrm{EtOH}(1: 1)$ ), ninhydrin reagent (1.5 g ninhydrin in $15 \mathrm{ml}$ acetic acid and $500 \mathrm{ml}$ $\mathrm{MeOH})$ or $\mathrm{CAM}$ reagent $\left(1.0 \mathrm{~g} \mathrm{Ce}\left(\mathrm{SO}_{4}\right)_{2} \cdot 4 \mathrm{H}_{2} \mathrm{O}\right.$ and $2.5 \mathrm{~g}$ ammonium molybdate pentahydrate in $96 \mathrm{ml}$ of $\mathrm{H}_{2} \mathrm{O}$ and $6 \mathrm{ml}$ of concentrated $\left.\mathrm{H}_{2} \mathrm{SO}_{4}\right)$ upon heating or via $\mathrm{UV}$ adsorption $(\lambda=254 \mathrm{~nm})$. NMR experiments were conducted on a OneNMR $400 \mathrm{MHz}$ or $600 \mathrm{MHz}$ spectrometer (Agilent). Chemical shifts were referenced to the internal standards $\mathrm{CHCl}_{3}\left(\delta\left({ }^{1} \mathrm{H}\right)=7.26 \mathrm{ppm}\right.$ and $\left.\delta\left({ }^{13} \mathrm{C}\right)=77.1 \mathrm{ppm}\right), \mathrm{H}_{2} \mathrm{O}$ $\left(\delta\left({ }^{1} \mathrm{H}\right)=7.26 \mathrm{ppm}\right), \mathrm{MeOH}\left(\delta\left({ }^{1} \mathrm{H}\right)=4.87 \mathrm{ppm}, \delta\left({ }^{13} \mathrm{C}\right)=49.0 \mathrm{ppm}\right)$ and trifluoroacetic acid $\left(\delta\left({ }^{19} \mathrm{~F}\right)=\right.$ $76.55 \mathrm{ppm}$ ). Coupling constants are reported in $\mathrm{Hz}$ and coupling patterns are indicated as s for singlet, $\mathrm{d}$ for doublets, dd for doublets of doublets, ddd for doublets of doublets of doublets, $t$ for triplets, $d t$ for doublets of triplets, td for triplet of doublets, $q$ for quartets and $m$ for multiplets. Signals were assigned by means of COSY, TOCSY and ${ }^{13} \mathrm{C}$ HSQC NMR experiments. Stereoselectivity at the anomeric position of the mannose scaffold was analyzed by measuring ${ }^{1} \mathrm{~J}_{\mathrm{Cl}, \mathrm{H} 1}$ coupling constants for $\mathbf{3}$, 4 and 7 (18). NMR spectra were processed with MestReNova (19). ESI-MS analysis was conducted using an 1100 Series LC/MS coupled to a G1946D ESI-Q spectrometer (Agilent). HR ESI-MS analysis was conducted using a 6210 ESI-TOF spectrometer (Agilent) or an Acquity H-Class UPLC/MS coupled to a Xevo G2-S ESI-Q-TOF spectrometer (Waters). Analytical HPLC was performed on a 1200 Series LC/MS coupled to a 6130 ESI-Q spectrometer (Agilent) using an analytical HyperCarb column (Thermo Scientific). Preparative HPLC was performed on a 1200 Series LC/MS using a semi-preparative HyperCarb column (Thermo Scientific). 
233

234

235

236

237

238

239

240

241

242

243

244

245

246

247

248

249

250

251

252

253

254

255

256

257

258

259

260

261

262

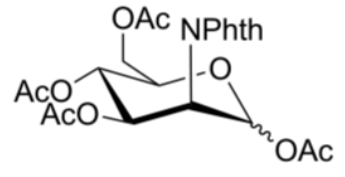

2

2 was prepared as previously published (20). Mannosamine hydrochloride (Dextra) (2.15 g, $10 \mathrm{mmol}$ ), phthalic anhydride $(1.63 \mathrm{~g}, 11 \mathrm{mmol})$ and pyridine $(2 \mathrm{ml}, 25 \mathrm{mmol})$ were dissolved in a mixture of acetone- $\mathrm{H}_{2} \mathrm{O}(1: 1,15 \mathrm{ml})$ and stirred at $50^{\circ} \mathrm{C}$ for $3 \mathrm{~h}$. Progress of the reaction was monitored by analytical TLC (propan-1-ol: ethyl acetate: $\mathrm{H}_{2} \mathrm{O}: 25 \%$ aqueous ammonia (6:3:1:1)). Solvents were evaporated in vacuo and acetic anhydride $(14.1 \mathrm{ml}, 150 \mathrm{mmol})$ and pyridine $(40 \mathrm{ml})$ were added to the residue. The mixture was heated to $50^{\circ} \mathrm{C}$ and stirred for $5 \mathrm{~h}$. Progress of the reaction was monitored by analytical TLC (toluene: ethyl acetate (4:1)). Solvents were evaporated in vacuo and the residue was taken up in chloroform $(250 \mathrm{ml})$. The organic phase was extracted with $1 \mathrm{M} \mathrm{HCl}$, saturated $\mathrm{NaHCO}_{3}$ and $\mathrm{H}_{2} \mathrm{O}$. Subsequently, the organic phase was dried with $\mathrm{MgSO}_{4}$. Solvents were evaporated in vacuo and the residue was purified via column chromatography (toluene: ethyl acetate (8:1)) to afford an $\alpha / \beta$ anomer mixture of $2(3.10 \mathrm{~g}, 6.50 \mathrm{mmol}, 65 \%)$ as a white solid.

${ }^{1} \mathrm{H}$ NMR (400.0 MHz, $\mathrm{CDCl}_{3}, \alpha$-anomer): $\delta=7.92-7.75$ ppm, m, $4 \mathrm{H}$ (aromatic $\mathrm{H}$ of Phth); $\delta=6.59$ ppm, d, $1 \mathrm{H}(\mathrm{H} 1) ; \delta=5.55$ ppm, dd, $1 \mathrm{H}, \mathrm{J}=6.8,8.2 \mathrm{~Hz}(\mathrm{H} 4) ; \delta=5.50 \mathrm{ppm}, \mathrm{dd}, 1 \mathrm{H}, \mathrm{J}=5.3,6.8 \mathrm{~Hz}$ (H3); $\delta=4.89, \mathrm{dd}, 1 \mathrm{H}, \mathrm{J}=3.9,5.3 \mathrm{~Hz}(\mathrm{H} 2) ; \delta=4.45 \mathrm{ppm}, \mathrm{dd}, 1 \mathrm{H}, \mathrm{J}=6.0,12.2 \mathrm{~Hz},(\mathrm{H} 6 \mathrm{a}) ; \delta=4.31$, dd, $1 \mathrm{H}, \mathrm{J}=3.3,12.2 \mathrm{ppm}(\mathrm{H} 6 \mathrm{~b}) ; \delta=4.23 \mathrm{ppm}$, ddd, $1 \mathrm{H}, \mathrm{J}=3.3,6.0,8.3 \mathrm{~Hz},(\mathrm{H} 5) ; \delta=2.15 \mathrm{ppm}, \mathrm{s}, 3$ $\mathrm{H}\left(\mathrm{OCOCH}_{3}\right) ; \delta=2.13$ ppm, s, $3 \mathrm{H}\left(\mathrm{OCOCH}_{3}\right) ; \delta=2.10 \mathrm{ppm}, \mathrm{s}, 3 \mathrm{H}\left(\mathrm{OCOCH}_{3}\right) ; \delta=1.96 \mathrm{ppm}, \mathrm{s}, 3 \mathrm{H}$ $\left(\mathrm{OCOCH}_{3}\right)$.

${ }^{13} \mathrm{C} \mathrm{NMR}\left(100.6 \mathrm{MHz}, \mathrm{CDCl}_{3}, \alpha\right.$-anomer $): \delta=170.8 \mathrm{ppm}, 1 \mathrm{C}\left(\mathrm{OCOCH}_{3}\right) ; \delta=169.9,1 \mathrm{C}\left(\mathrm{OCOCH}_{3}\right)$; $\delta=169.5 \mathrm{ppm}\left(\mathrm{OCOCH}_{3}\right) ; \delta=168.5 \mathrm{ppm}, 1 \mathrm{C}\left(\mathrm{OCOCH}_{3}\right) ; \delta=167.6 \mathrm{ppm}, 2 \mathrm{C}$ (carbonyl C of Phth); $\delta$ $=134.5$ ppm, $2 \mathrm{C}$ (aromatic $\mathrm{C}$ of Phth); $\delta=131.3 \mathrm{ppm}, 2 \mathrm{C}$ (aromatic $\mathrm{C}$ of Phth); $\delta=123.8 \mathrm{ppm}, 2 \mathrm{C}$ (aromatic C of Phth); $\delta=90.4$ ppm, $1 \mathrm{C}(\mathrm{C} 1) ; \delta=71.1 \mathrm{ppm}, 1 \mathrm{C}(\mathrm{C} 5) ; \delta=69.0 \mathrm{ppm}, 1 \mathrm{C}(\mathrm{C} 3) ; \delta=$ $67.8 \mathrm{ppm}, 1 \mathrm{C}(\mathrm{C} 4) ; \delta=62.5 \mathrm{ppm}, 1 \mathrm{C}(\mathrm{C} 6) ; \delta=50.5,1 \mathrm{C}(\mathrm{C} 2) ; \delta=21.0 \mathrm{ppm}, 1 \mathrm{C}\left(\mathrm{OCOCH}_{3}\right) ; \delta=$ 20.8 ppm, $2 \mathrm{C}$ (two times $\left.\mathrm{OCOCH}_{3}\right) ; \delta=20.7$ ppm, $1 \mathrm{C}\left(\mathrm{OCOCH}_{3}\right)$.

${ }^{1} \mathrm{H}$ NMR (400.0 MHz, $\mathrm{CDCl}_{3}, \beta$-anomer): $\delta=7.92-7.75$ ppm, m, $4 \mathrm{H}$ (aromatic $\mathrm{H}$ of Phth); $\delta=6.06$ ppm, t, $1 \mathrm{H}, \mathrm{J}=9.5,9.5 \mathrm{~Hz}(\mathrm{H} 4) ; \delta=5.99 \mathrm{ppm}, \mathrm{d}, 1 \mathrm{H}, \mathrm{J}=2.8 \mathrm{~Hz}(\mathrm{H} 1) ; \delta=5.37 \mathrm{ppm}, \mathrm{dd}, 1 \mathrm{H}, \mathrm{J}=$ 6.8, $9.4 \mathrm{~Hz}(\mathrm{H} 3) ; \delta=5.06 \mathrm{ppm}, \mathrm{dd}, \mathrm{J}=2.8,6.8 \mathrm{~Hz}(\mathrm{H} 2) ; \delta=4.48 \mathrm{ppm}$, dd, $1 \mathrm{H}, \mathrm{J}=6.0 \mathrm{ppm}, 12.2$ (H6a); $\delta=4.27$, dd, $1 \mathrm{H}, \mathrm{J}=2.3,12.2 \mathrm{ppm}(\mathrm{H} 6 \mathrm{~b}) ; \delta=3.93$ ppm, ddd, $1 \mathrm{H}, \mathrm{J}=2.3,6.0,9.7$ (H5); $\delta=$ 2.16 ppm, s, $3 \mathrm{H}\left(\mathrm{OCOCH}_{3}\right) ; \delta=2.07$ ppm, s, $3 \mathrm{H}\left(\mathrm{OCOCH}_{3}\right) ; \delta=1.97 \mathrm{ppm}$, s, $3 \mathrm{H}\left(\mathrm{OCOCH}_{3}\right) ; \delta=$ $1.91 \mathrm{ppm}, \mathrm{s}, 3 \mathrm{H}\left(\mathrm{OCOCH}_{3}\right)$. 
$263{ }^{13} \mathrm{C} \mathrm{NMR}\left(100.6 \mathrm{MHz}, \mathrm{CDCl}_{3}, \beta\right.$-anomer): $\delta=170.7 \mathrm{ppm}, 1 \mathrm{C}\left(\mathrm{OCOCH}_{3}\right) ; \delta=169.7,1 \mathrm{C}\left(\mathrm{OCOCH}_{3}\right)$; $264 \delta=169.5 \mathrm{ppm}, 1 \mathrm{C}(\mathrm{OCOCH} 3) ; \delta=168.4 \mathrm{ppm}, 1 \mathrm{C}\left(\mathrm{OCOCH}_{3}\right) ; \delta=168.0 \mathrm{ppm}, 2 \mathrm{C}$ (carbonyl C of 265 Phth); $\delta=134.3$ ppm, 2 C (aromatic C of Phth); $\delta=131.2$ ppm, 2 C (aromatic C of Phth); $\delta=123.6$ 266 ppm, $2 \mathrm{C}$ (aromatic C of Phth); $\delta=90.3 \mathrm{ppm}, 1 \mathrm{C}(\mathrm{C} 1) ; \delta=73.9 \mathrm{ppm}, 1 \mathrm{C}(\mathrm{C} 5) ; \delta=69.8 \mathrm{ppm}, 1 \mathrm{C}$ $267(\mathrm{C} 3) ; \delta=66.7 \mathrm{ppm}, 1 \mathrm{C}(\mathrm{C} 4) ; \delta=62.5 \mathrm{ppm}, 1 \mathrm{C}(\mathrm{C} 6) ; \delta=49.9,1 \mathrm{C}(\mathrm{C} 2) ; \delta=20.7 \mathrm{ppm}, 2 \mathrm{C}$ (two 268 times $\left.\mathrm{OCOCH}_{3}\right) ; \delta=20.5 \mathrm{ppm}, 1 \mathrm{C}\left(\mathrm{OCOCH}_{3}\right) ; \delta=20.4 \mathrm{ppm}, 1 \mathrm{C}\left(\mathrm{OCOCH}_{3}\right)$.

$269 \quad \mathrm{R}_{\mathrm{f}}=0.35$ with toluene:ethyl acetate $(2: 1)$.

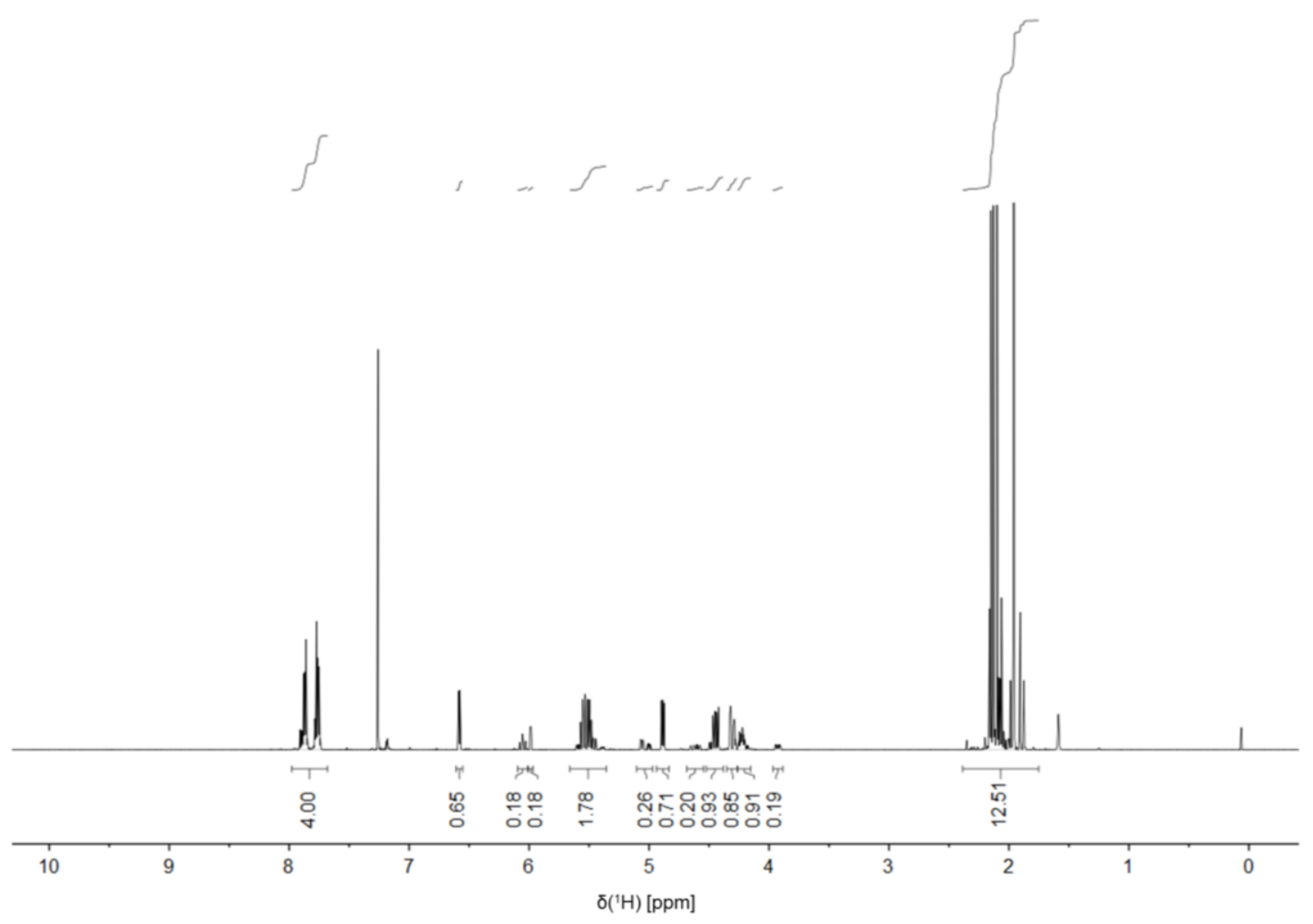




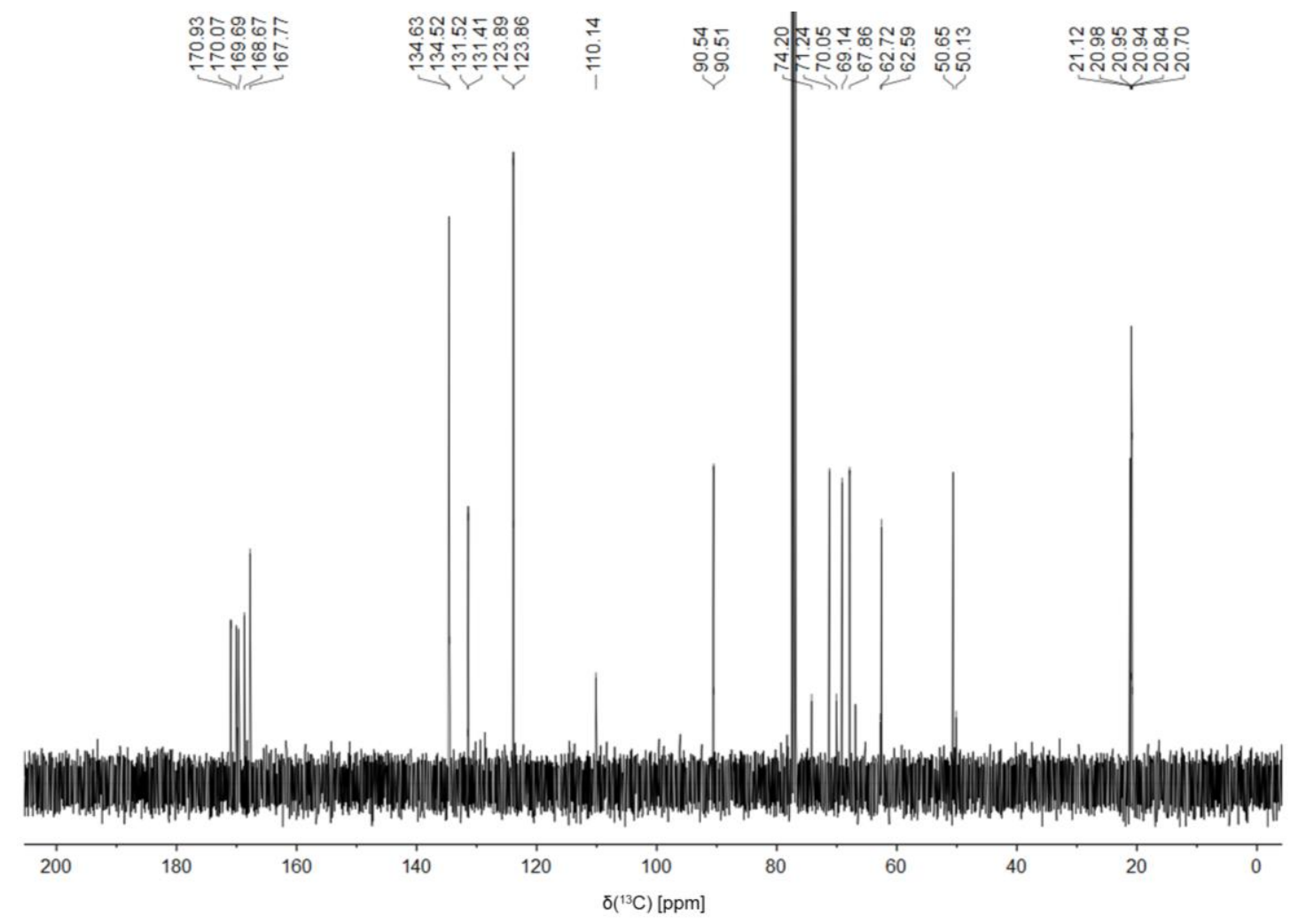

271

272 
274

275

276

277

278

279

280

281

282

283

284

285

286

287

288

289

290

291

292

293

294

295

296

297

298

299

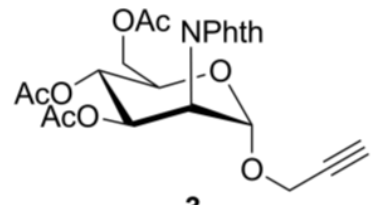

3

2 (1.40 g, $2.9 \mathrm{mmol}$ ) was dissolved in anhydrous DCM:ether (29 $\mathrm{ml}, 2: 1)$. The mixture was stirred at $0^{\circ} \mathrm{C}$ and kept under argon. Propargyl alcohol $(700 \mu \mathrm{l}, 11.7 \mathrm{mmol})$ and $\mathrm{BF}_{3} \cdot \mathrm{OEt}_{2}(740 \mu \mathrm{l}, 5.9 \mathrm{mmol})$ were added, the reaction was allowed to heat up to room temperature and stirred for $64 \mathrm{~h}$. Progress of the reaction was monitored by analytical TLC (toluene:ethyl acetate (2:1)). The mixture was diluted in DCM $(200 \mathrm{ml})$ and the organic phase was extracted with saturated $\mathrm{NaHCO}_{3}$ and $\mathrm{H}_{2} \mathrm{O}$. Subsequently, the organic phase was dried with $\mathrm{MgSO}_{4}$. Solvents were evaporated in vacuo and the residue was purified via column chromatography (toluene:ethyl acetate (8:1)) to yield $\mathbf{3}$ (752 $\mathrm{mg}, 1.57 \mathrm{mmol}, 54 \%$ ) as a light yellow resin. A stereoselectivity of 10 to 1 favoring the $\alpha$-anomer was determined via ${ }^{1} \mathrm{H}$ NMR experiments of the crude mixture. Starting material 2 that was not converted was recovered.

${ }^{1} \mathrm{H}$ NMR (400.0 MHz, $\mathrm{CDCl}_{3}$ ): $\delta=7.88-7.72 \mathrm{ppm}, \mathrm{m}, 4 \mathrm{H}$ (aromatic $\mathrm{H}$ of Phth); $\delta=5.58 \mathrm{ppm}, \mathrm{d}, 1 \mathrm{H}$, $\mathrm{J}=4.1 \mathrm{~Hz}(\mathrm{H} 1) ; \delta=5.48 \mathrm{ppm}, \mathrm{dd}, 1 \mathrm{H}, \mathrm{J}=6.5,8.0 \mathrm{~Hz}(\mathrm{H} 4) ; \delta=5.45 \mathrm{ppm}, \mathrm{dd}, 1 \mathrm{H}, \mathrm{J}=4.9,6.4 \mathrm{~Hz}$ (H3); $\delta=4.87 \mathrm{ppm}, \mathrm{dd}, 1 \mathrm{H}, \mathrm{J}=4.0 \mathrm{~Hz}, 4.9 \mathrm{~Hz}(\mathrm{H} 2) ; \delta=4.46 \mathrm{ppm}, \mathrm{dd}, 1 \mathrm{H}, \mathrm{J}=6.1,12.1 \mathrm{~Hz}(\mathrm{H} 6 \mathrm{a}) ; \delta$ $=4.28 \mathrm{ppm}, \mathrm{dd}, 2 \mathrm{H}, \mathrm{J}=0.5,2.4 \mathrm{~Hz}\left(\mathrm{OCH}_{2} \mathrm{CCH}\right) ; \delta=4.27 \mathrm{ppm}, \mathrm{dd}, 1 \mathrm{H}, \mathrm{J}=3.0,12.1 \mathrm{~Hz}(\mathrm{H6b}) ; \delta=$ $4.17 \mathrm{ppm}$, ddd, $1 \mathrm{H}, \mathrm{J}=3.0,6.2,8,0 \mathrm{~Hz}(\mathrm{H} 5) ; \delta=2.41, \mathrm{t}, 1 \mathrm{H}, \mathrm{J}=2.4 \mathrm{~Hz}\left(\mathrm{OCH}_{2} \mathrm{CCH}\right) ; \delta=2.16 \mathrm{ppm}$, s, $3 \mathrm{H}\left(\mathrm{OCOCH}_{3}\right) ; \delta=2.09$ ppm, s, $3 \mathrm{H}\left(\mathrm{OCOCH}_{3}\right) ; \delta=1.95 \mathrm{ppm}, \mathrm{s}, 3 \mathrm{H}\left(\mathrm{OCOCH}_{3}\right)$.

${ }^{13} \mathrm{C} \mathrm{NMR}(100.6 \mathrm{MHz}, \mathrm{CDCl} 3): \delta=170.9 \mathrm{ppm}, 1 \mathrm{C}\left(\mathrm{OCOCH}_{3}\right) ; \delta=170.0 \mathrm{ppm}, 1 \mathrm{C}\left(\mathrm{OCOCH}_{3}\right) ; \delta=$ 169.8 ppm, $1 \mathrm{C}\left(\mathrm{OCOCH}_{3}\right) ; \delta=168.0$ ppm, $2 \mathrm{C}$ (carbonyl C of Phth); $\delta=134.5 \mathrm{ppm}, 2 \mathrm{C}$ (aromatic C of Phth); $\delta=131.5$ ppm, $2 \mathrm{C}$ (aromatic C of Phth); $\delta=123.7$ ppm, $2 \mathrm{C}$ (aromatic C of Phth); $\delta=95.5$ ppm, $1 \mathrm{C}(\mathrm{C} 1) ; \delta=78.5 \mathrm{ppm}, 1 \mathrm{C}\left(\mathrm{OCH}_{2} \mathrm{CCH}\right) ; \delta=75.4 \mathrm{ppm}, 1 \mathrm{C}\left(\mathrm{OCH}_{2} \mathrm{CCH}\right) ; \delta=69.6 \mathrm{ppm}, 1 \mathrm{C}$ (C5); $\delta=69.5 \mathrm{ppm}, 1 \mathrm{C}(\mathrm{C} 3) ; \delta=68.3 \mathrm{ppm}, 1 \mathrm{C}(\mathrm{C} 4) ; \delta=62.8 \mathrm{ppm}, 1 \mathrm{C}(\mathrm{C} 6) ; \delta=54.9 \mathrm{ppm}, 1 \mathrm{C}$ $\left(\mathrm{OCH}_{2} \mathrm{CCH}\right) ; \delta=51.6 \mathrm{ppm}, 1 \mathrm{C}(\mathrm{C} 2) ; \delta=21.0 \mathrm{ppm}, 2 \mathrm{C}$ (two times $\left.\mathrm{OCOCH}_{3}\right) ; \delta=20.9 \mathrm{ppm}, 1 \mathrm{C}$ $\left(\mathrm{OCOCH}_{3}\right)$.

$\mathrm{R}_{\mathrm{f}}=0.47$ with toluene:ethyl acetate $(2: 1)$.

ESI-MS for $\mathrm{C}_{23} \mathrm{H}_{23} \mathrm{NO}_{10}: \mathrm{m} \cdot \mathrm{z}^{-1}\left(\mathrm{M}+\mathrm{Na}^{+}\right)_{\text {calc }}=496.1 ; \mathrm{m} \cdot \mathrm{z}^{-1}\left(\mathrm{M}+\mathrm{Na}^{+}\right)_{\mathrm{obs}}=496.0 ; \mathrm{m} \cdot \mathrm{z}^{-1}\left(\mathrm{M}+\mathrm{NH}_{4}^{+}\right)_{\text {calc }}=$ 491.2; $\mathrm{m} \cdot \mathrm{z}^{-1}\left(\mathrm{M}+\mathrm{NH}_{4}{ }^{+}\right)_{\mathrm{obs}}=491.2$. 


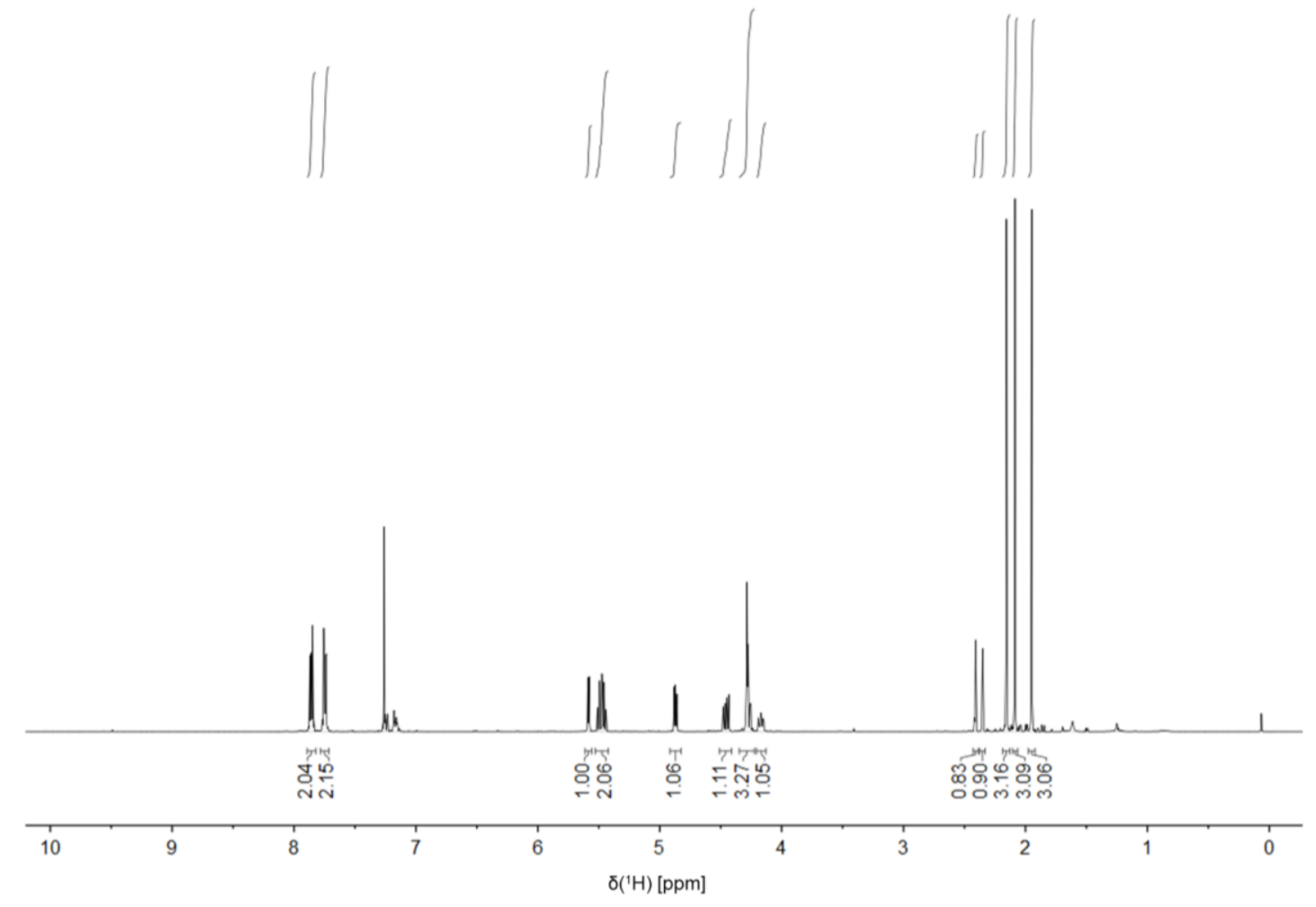

300

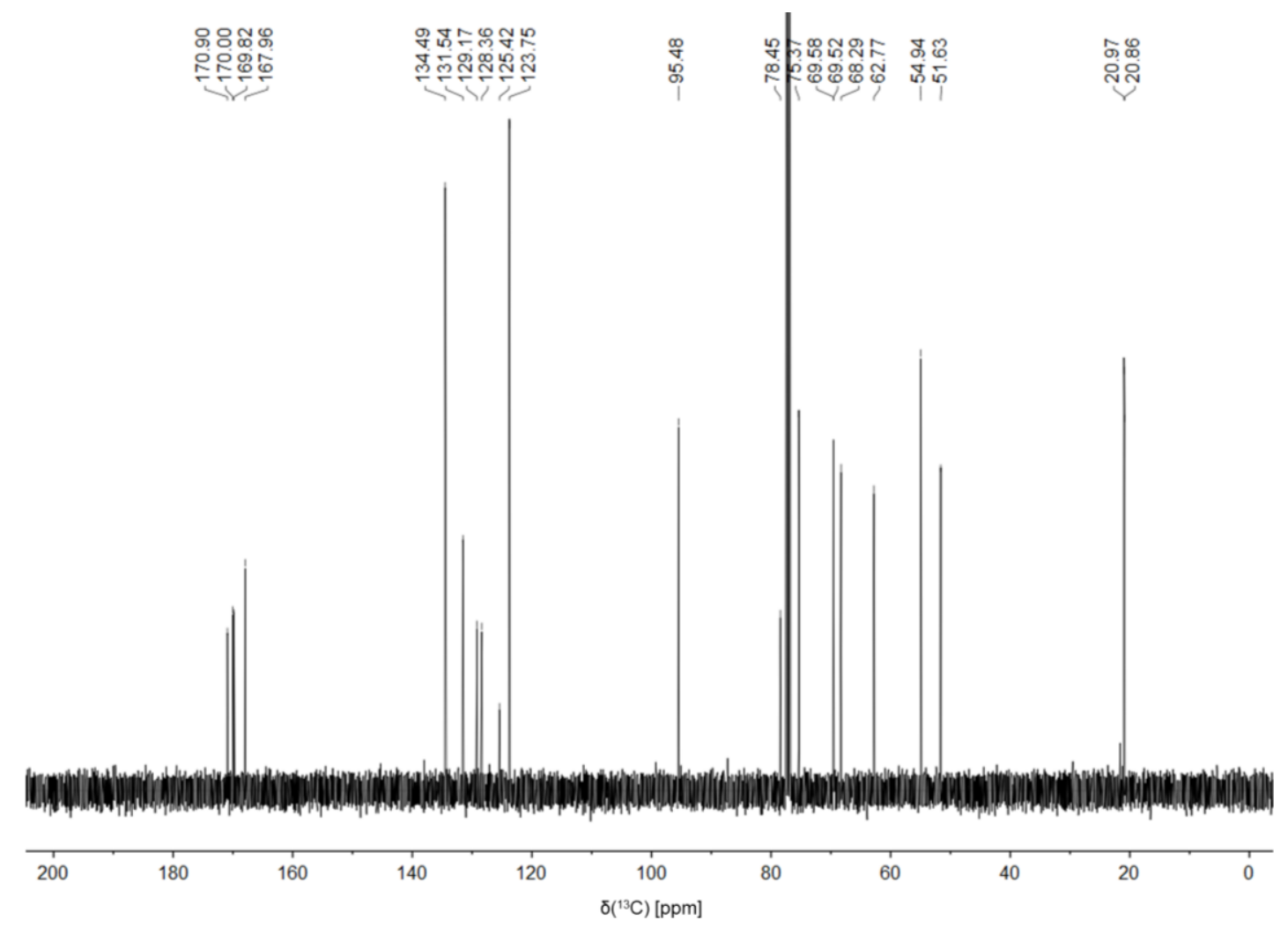

301

302 
304

305

306

307

308

309

310

311

312

313

314

315

316

317

318

319

320

321

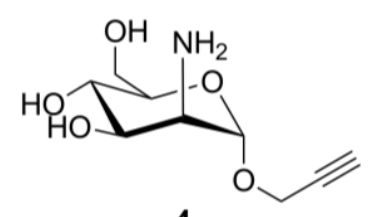

4

$3(2.70 \mathrm{~g}, 5.6 \mathrm{mmol})$ was dissolved in $\mathrm{EtOH}$ containing $33 \%$ methylamine $(110 \mathrm{ml})$ at room temperature. The mixture was stirred overnight and progress of the reaction was monitored by analytical TLC (20\% MeOH in DCM). Solvents were evaporated in vacuo and the residue was purified via column chromatography (gradient: hexane, hexane:DCM (1:1), DCM, 1\% $\mathrm{MeOH}$ in DCM, $5 \% \mathrm{MeOH}$ in DCM and elution with $20 \% \mathrm{MeOH}$ in DCM). Silica gel particles were removed by filtration in $\mathrm{MeOH}$ with a cellulose acetate membrane at a pore size $0.2 \mu \mathrm{m}$ to yield 4 (950 mg, $4.40 \mathrm{mmol}, 78 \%$ ) as a white solid.

${ }^{1} \mathrm{H}$ NMR (400.0 MHz, MeOD): $\delta=4.95$ ppm, d, $1 \mathrm{H}, \mathrm{J}=1.3 \mathrm{~Hz}(\mathrm{H} 1) ; \delta=4.26 \mathrm{ppm}, \mathrm{d}, 2 \mathrm{H}, \mathrm{J}=2.4$ $\mathrm{Hz}\left(\mathrm{OCH}_{2} \mathrm{CCH}\right) ; \delta=3.79 \mathrm{ppm}, \mathrm{dd}, 1 \mathrm{H}, \mathrm{J}=2.4,11.9 \mathrm{~Hz}(\mathrm{H} 6 \mathrm{a}) ; \delta=3.76 \mathrm{ppm}, \mathrm{dd}, 1 \mathrm{H}, \mathrm{J}=4.5,9.3 \mathrm{~Hz}$ $(\mathrm{H} 3) ; \delta=3.73 \mathrm{ppm}, \mathrm{dd}, 1 \mathrm{H}, \mathrm{J}=4.7,11.8 \mathrm{~Hz}(\mathrm{H} 6 \mathrm{~b}) ; \delta=3.57 \mathrm{ppm}, \mathrm{m}, 1 \mathrm{H}(\mathrm{H} 4) ; \delta=3.50 \mathrm{ppm}$, ddd, 1 $\mathrm{H}, \mathrm{J}=2.3,4.8,9.8 \mathrm{~Hz}(\mathrm{H} 5), \delta=3.05 \mathrm{ppm}, \mathrm{dd}, 1 \mathrm{H}, \mathrm{J}=1.4,4.3 \mathrm{~Hz}(\mathrm{H} 2) ; \delta=2.85 \mathrm{ppm}, \mathrm{t}, 1 \mathrm{H}, \mathrm{J}=2.5$ $\mathrm{Hz}\left(\mathrm{OCH}_{2} \mathrm{CCH}\right)$.

${ }^{13} \mathrm{C}$ NMR (100.6 MHz, MeOD): $\delta=100.0$ ppm, $1 \mathrm{C}(\mathrm{C} 1) ; \delta=80.0 \mathrm{ppm}, 1 \mathrm{C}\left(\mathrm{OCH}_{2} \mathrm{CCH}\right) ; \delta=76.0$ ppm, $1 \mathrm{C}\left(\mathrm{OCH}_{2} \mathrm{CCH}\right) ; \delta=74.7 \mathrm{ppm}, 1 \mathrm{C}(\mathrm{C} 5) ; \delta=71.8 \mathrm{ppm}, 1 \mathrm{C}(\mathrm{C} 3) ; \delta=67.7 \mathrm{ppm}, 1 \mathrm{C}(\mathrm{C} 4) ; \delta=$ $62.3 \mathrm{ppm}, 1 \mathrm{C}(\mathrm{C} 6) ; \delta=55.7 \mathrm{ppm}, 1 \mathrm{C}(\mathrm{C} 2) ; \delta=54.9 \mathrm{ppm}, 1 \mathrm{C}\left(\mathrm{OCH}_{2} \mathrm{CCH}\right)$.

$\mathrm{R}_{\mathrm{f}}=0.22$ with $20 \% \mathrm{MeOH}$ in DCM.

HR ESI-MS for $\mathrm{C}_{9} \mathrm{H}_{15} \mathrm{NO}_{5}: \mathrm{m} \cdot \mathrm{z}^{-1}\left(\mathrm{M}+\mathrm{Na}^{+}\right)_{\text {calc }}=240.085, \mathrm{~m} \cdot \mathrm{z}^{-1}\left(\mathrm{M}+\mathrm{Na}^{+}\right)_{\text {obs }}=240.085$. 


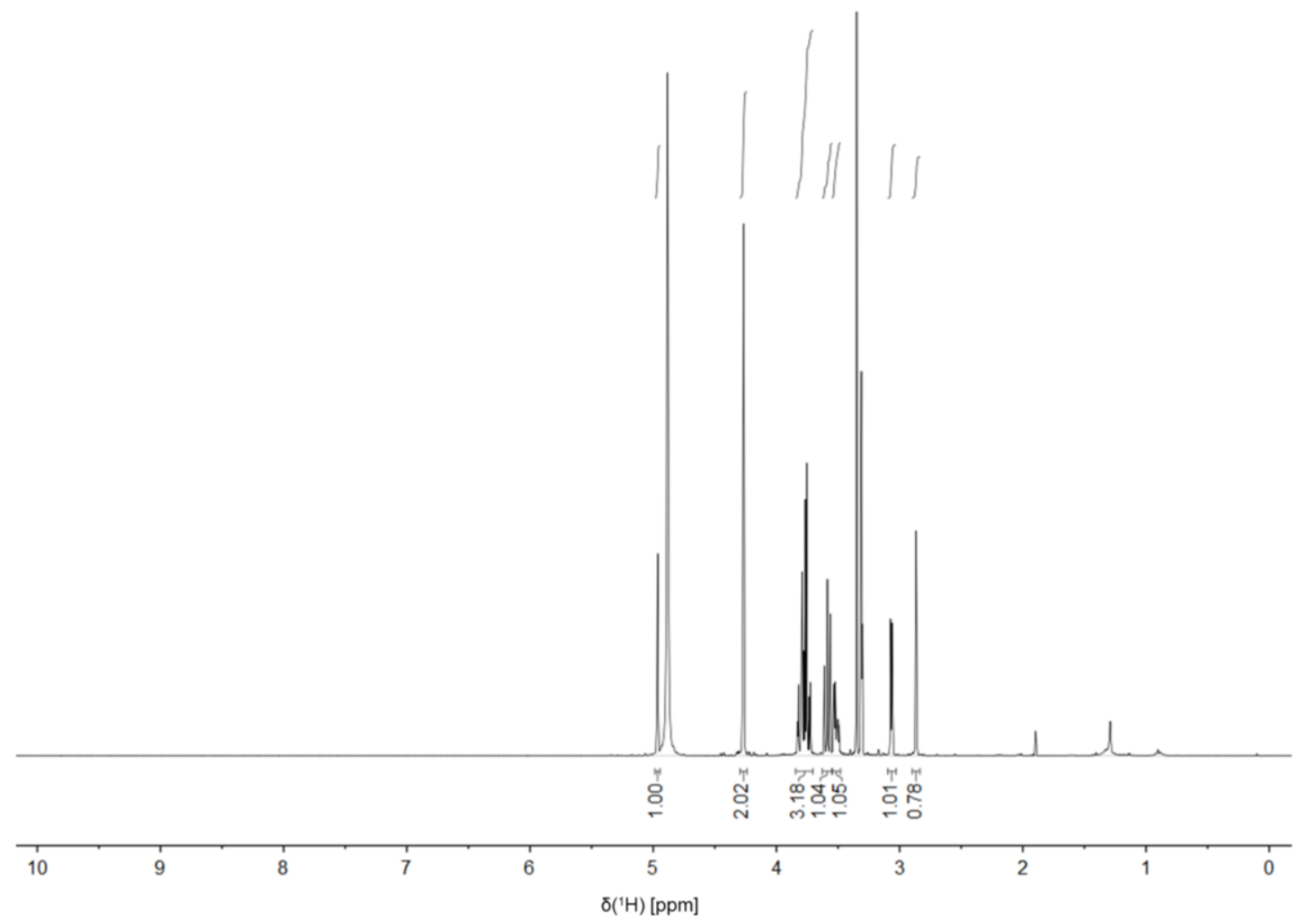

322

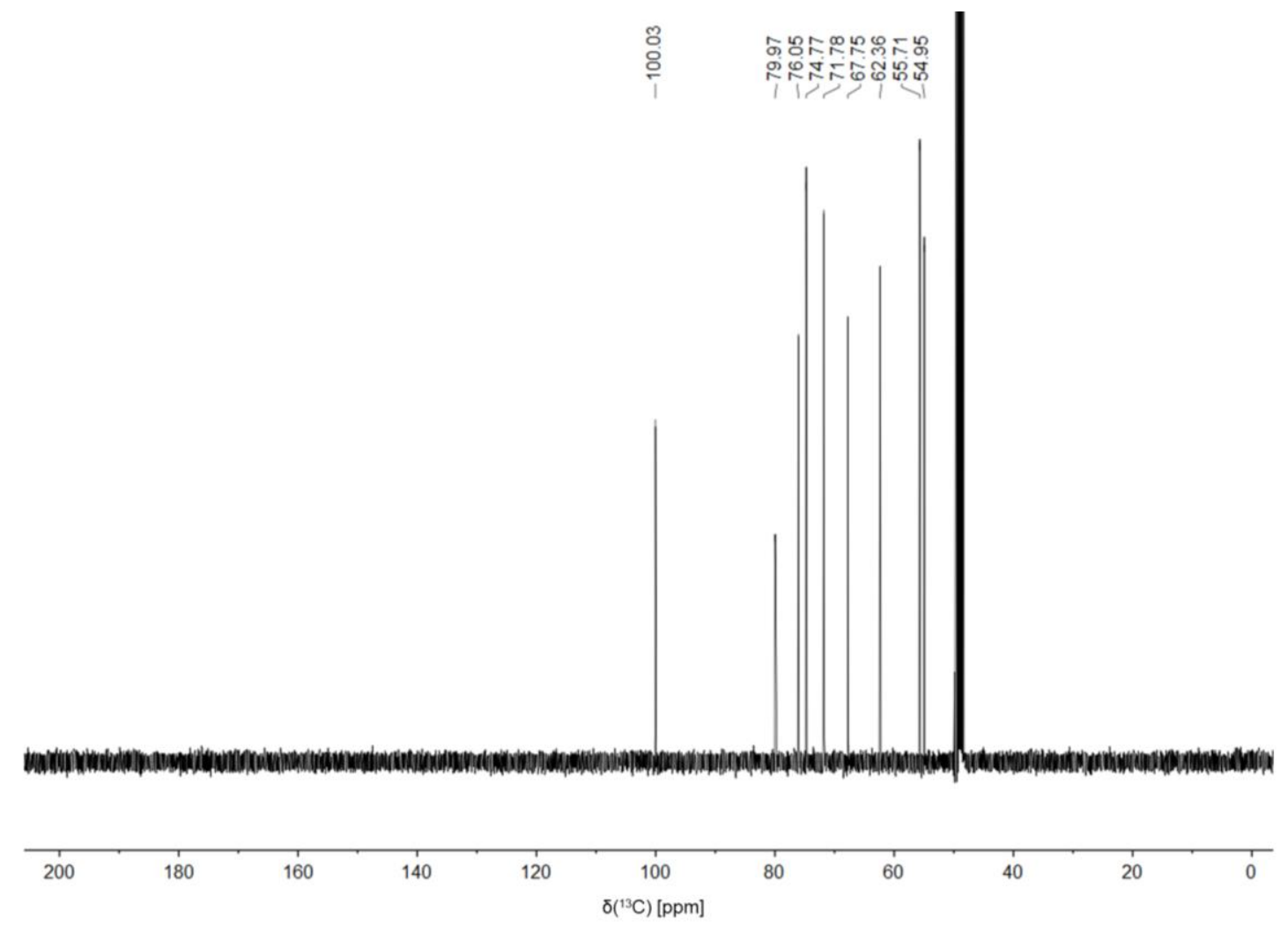

323

324 
326

327

328

329

330

331

332

333

334

335

336

337

338

339

340

341

342

343

344

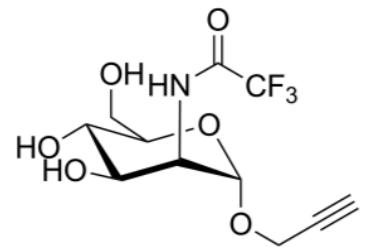

5.1

4 (255 mg, $1.2 \mathrm{mmol}$ ) was dissolved in DMF (2.4 ml) and ethyl 2,2,2-trifluoroacetate (155 $\mu 1,1.3$ mmol) was added at room temperature. Progress of the reaction was monitored by analytical TLC (10\% $\mathrm{MeOH}$ in DCM). After 6 hours solvents were evaporated in vacuo and the residue was purified via column chromatography (gradient: DCM and elution with $\mathrm{ml} 5 \% \mathrm{MeOH}$ in DCM). Silica gel particles were removed by filtration in $\mathrm{MeOH}$ with a cellulose acetate membrane at a pore size $0.2 \mu \mathrm{m}$ to yield $\mathbf{5 . 1}$ (248 $\mathrm{mg}, 790 \mu \mathrm{mol}, 67 \%)$ as a white solid.

${ }^{1} \mathrm{H}$ NMR (600.0 MHz, MeOD): $\delta=4.94 \mathrm{ppm}, \mathrm{d}, 1 \mathrm{H}, \mathrm{J}=0.8 \mathrm{~Hz}(\mathrm{H} 1) ; \delta=4.39 \mathrm{ppm}, \mathrm{dd}, 1 \mathrm{H}, \mathrm{J}=0.8$, $4.9 \mathrm{~Hz}(\mathrm{H} 2) ; \delta=4.27$ ppm, m, $2 \mathrm{H}\left(\mathrm{OCH}_{2} \mathrm{CCH}\right) ; \delta=3.96 \mathrm{ppm}$, dd, $1 \mathrm{H}, \mathrm{J}=4.8,9.7 \mathrm{~Hz}(\mathrm{H} 3) ; \delta=3.87$ ppm, dd, $2 \mathrm{H}, \mathrm{J}=4.0,11.7 \mathrm{~Hz}(\mathrm{H} 6 \mathrm{a}) ; \delta=3.79$ ppm, dd, $2 \mathrm{H}, \mathrm{J}=2.4,11.7 \mathrm{~Hz}(\mathrm{H} 6 \mathrm{~b}) ; \delta=3.65$ ppm, t, 1 $\mathrm{H}, \mathrm{J}=9.8 \mathrm{~Hz}(\mathrm{H} 4) ; \delta=3.57 \mathrm{ppm}$, ddd, $1 \mathrm{H}, \mathrm{J}=2.2,3.8,9.9 \mathrm{~Hz}(\mathrm{H} 5) ; \delta=2.88 \mathrm{ppm}, \mathrm{t}, 1 \mathrm{H}, \mathrm{J}=2.5 \mathrm{~Hz}$ $\left(\mathrm{OCH}_{2} \mathrm{CCH}\right)$.

${ }^{13} \mathrm{C}$ NMR (100.6 MHz, MeOD): $\delta=159.6 \mathrm{ppm}, \mathrm{q}, 1 \mathrm{C}, \mathrm{J}=37.6 \mathrm{~Hz}\left(\mathrm{NHCOCF}_{3}\right) ; \delta=117.3 \mathrm{ppm}, \mathrm{q}$, $1 \mathrm{C}, \mathrm{J}=286.6 \mathrm{~Hz}\left(\mathrm{NHCOCF}_{3}\right) ; \delta=98.5 \mathrm{ppm}, 1 \mathrm{C}(\mathrm{C} 1) ; \delta=79.6 \mathrm{ppm}, 1 \mathrm{C}\left(\mathrm{OCH}_{2} \mathrm{CCH}\right) ; \delta=76.4 \mathrm{ppm}$, $1 \mathrm{C}\left(\mathrm{OCH}_{2} \mathrm{CCH}\right) ; \delta=74.5$ ppm, $1 \mathrm{C}(\mathrm{C} 5) ; \delta=70.5 \mathrm{ppm}, 1 \mathrm{C}(\mathrm{C} 3) ; \delta=67.5 \mathrm{ppm}, 1 \mathrm{C}(\mathrm{C} 4) ; \delta=61.5$ ppm, $1 \mathrm{C}(\mathrm{C} 6) ; \delta=55.3 \mathrm{ppm}, 1 \mathrm{C}\left(\mathrm{OCH}_{2} \mathrm{CCH}\right) ; \delta=54.8 \mathrm{ppm}, 1 \mathrm{C}(\mathrm{C} 2)$.

${ }^{19} \mathrm{~F}$ NMR $\left(376.0 \mathrm{MHz}, \mathrm{D}_{2} \mathrm{O}\right): \delta=-75.8 \mathrm{ppm}, \mathrm{s}, 3 \mathrm{~F}\left(\mathrm{CF}_{3}\right)$.

$\mathrm{R}_{\mathrm{f}}=0.39$ with $10 \%$ in $\mathrm{MeOH}$ in DCM.

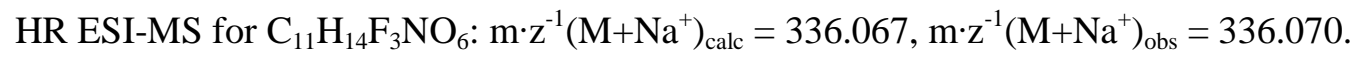




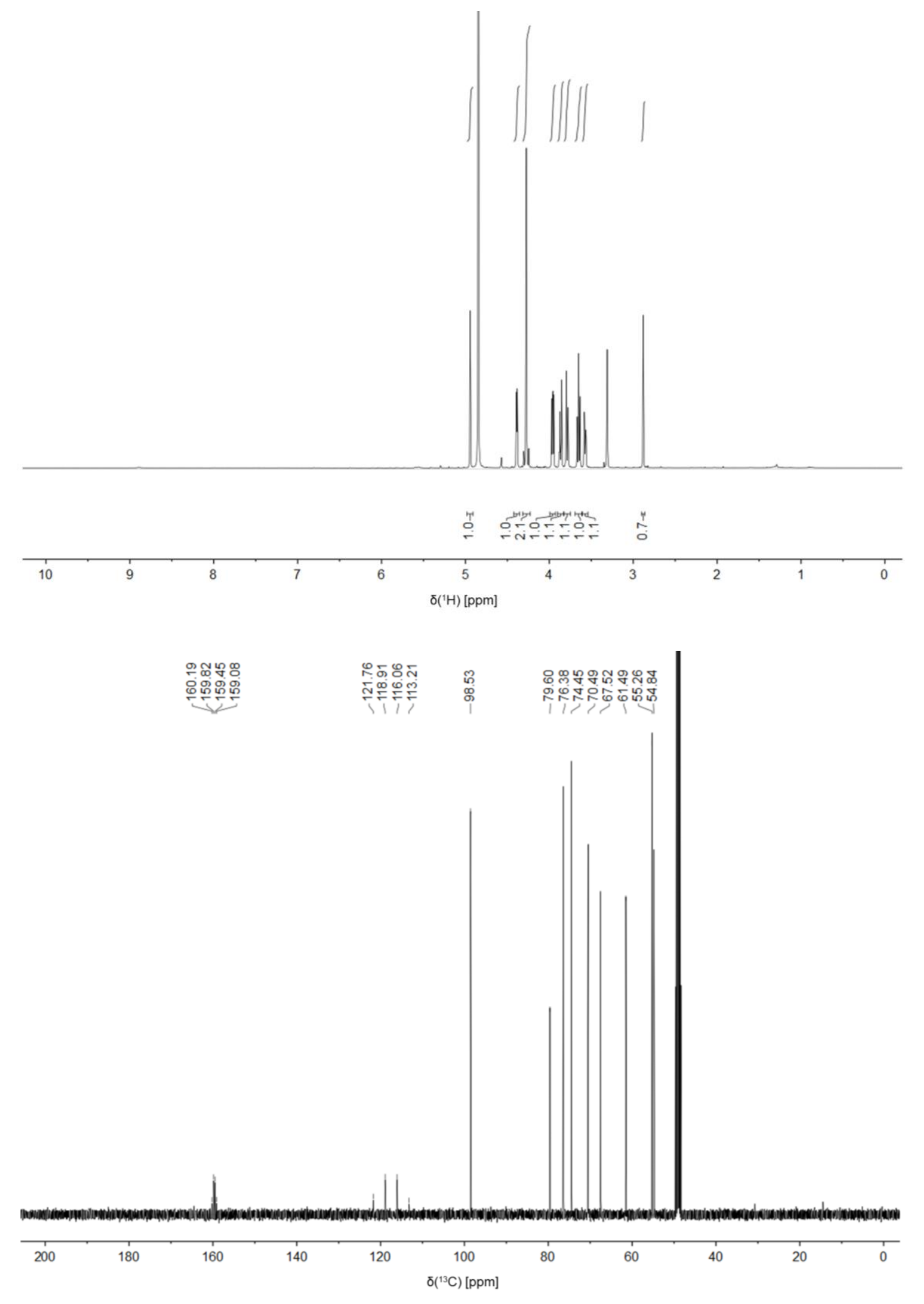




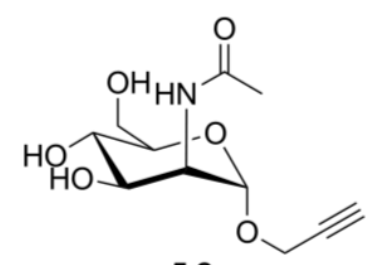

5.2

$3504(59 \mathrm{mg}, 270 \mu \mathrm{mol})$ was dissolved in $\mathrm{MeOH}(5 \mathrm{ml})$ and acetic anhydride (194 mg, $1.9 \mathrm{mmol})$ was 351 added. The mixture was stirred at room temperature overnight. Progress of the reaction was monitored 352 by analytical TLC (5\% MeOH in DCM). Solvents were evaporated in vacuo and the residue was 353 purified via column chromatography (gradient: hexane, hexane:DCM (1:1), DCM, 5\% MeOH in DCM and elution with $20 \% \mathrm{MeOH}$ in DCM). Silica gel particles were removed by filtration in $\mathrm{MeOH}$ with a cellulose acetate membrane at a pore size $0.2 \mu \mathrm{m}$ to yield $\mathbf{5 . 2}(57 \mathrm{mg}, 220 \mu \mathrm{mol}, 81 \%)$ as a white solid.

${ }^{1} \mathrm{H}$ NMR (400.0 MHz, D $\left.2 \mathrm{O}\right): \delta=5.00$ ppm, d, $1 \mathrm{H}, \mathrm{J}=1.1 \mathrm{~Hz}(\mathrm{H} 1) ; \delta=4.37 \mathrm{ppm}, \mathrm{dd}, 1 \mathrm{H}, \mathrm{J}=1.1,4.8$ $\mathrm{Hz}(\mathrm{H} 2) ; \delta=4.34 \mathrm{ppm}, \mathrm{m}, 2 \mathrm{H}\left(\mathrm{OCH}_{2} \mathrm{CCH}\right) ; \delta=4.02 \mathrm{ppm}, \mathrm{dd}, 1 \mathrm{H}, \mathrm{J}=4.8,9.4 \mathrm{~Hz}(\mathrm{H} 3) ; \delta=3.88$ ppm, m, $2 \mathrm{H}(\mathrm{H6a} / \mathrm{b}) ; \delta=3.71$ ppm, dt, $1 \mathrm{H}, \mathrm{J}=3.5,10.0 \mathrm{~Hz}(\mathrm{H} 5) ; \delta=3.66$ ppm, m, $1 \mathrm{H}(\mathrm{H} 4) ; \delta=$ $2.93 \mathrm{ppm}, \mathrm{t}, 1 \mathrm{H}, \mathrm{J}=2.5 \mathrm{~Hz}\left(\mathrm{OCH}_{2} \mathrm{CCH}\right) ; \delta=2.07 \mathrm{ppm}, \mathrm{s}, 3 \mathrm{H}\left(\mathrm{NHCOCH}_{3}\right)$.

${ }^{13} \mathrm{C} \mathrm{NMR}\left(100.6 \mathrm{MHz}, \mathrm{D}_{2} \mathrm{O}\right): \delta=175.4 \mathrm{ppm}, 1 \mathrm{C}\left(\mathrm{NHCOCH}_{3}\right) ; \delta=98.4 \mathrm{ppm}, 1 \mathrm{C}(\mathrm{C} 1) ; \delta=78.8 \mathrm{ppm}$, $1 \mathrm{C}\left(\mathrm{OCH}_{2} \mathrm{CCH}\right) ; \delta=76.2 \mathrm{ppm}, 1 \mathrm{C}\left(\mathrm{OCH}_{2} \mathrm{CCH}\right) ; \delta=73.3 \mathrm{ppm}, 1 \mathrm{C}(\mathrm{C} 5) ; \delta=69.6 \mathrm{ppm}, 1 \mathrm{C}(\mathrm{C} 3) ; \delta$ $=67.1 \mathrm{ppm}, 1 \mathrm{C}(\mathrm{C} 4) ; \delta=60.8 \mathrm{ppm}, 1 \mathrm{C}(\mathrm{C} 6) ; \delta=55.3 \mathrm{ppm}, 1 \mathrm{C}\left(\mathrm{OCH}_{2} \mathrm{CCH}\right) ; \delta=53.0 \mathrm{ppm}, 1 \mathrm{C}$ $(\mathrm{C} 2) ; \delta=22.5 \mathrm{ppm}, 1 \mathrm{C}\left(\mathrm{NHCOCH}_{3}\right)$.

$\mathrm{R}_{\mathrm{f}}=0.27$ with $10 \% \mathrm{MeOH}$ in DCM.

HR ESI-MS for $\mathrm{C}_{11} \mathrm{H}_{17} \mathrm{NO}_{6}: \mathrm{m} \cdot \mathrm{z}^{-1}\left(\mathrm{M}+\mathrm{Na}^{+}\right)_{\text {calc }}=282.095 ; \mathrm{m} \cdot \mathrm{z}^{-1}\left(\mathrm{M}+\mathrm{Na}^{+}\right)_{\text {obs }}=282.095$. 

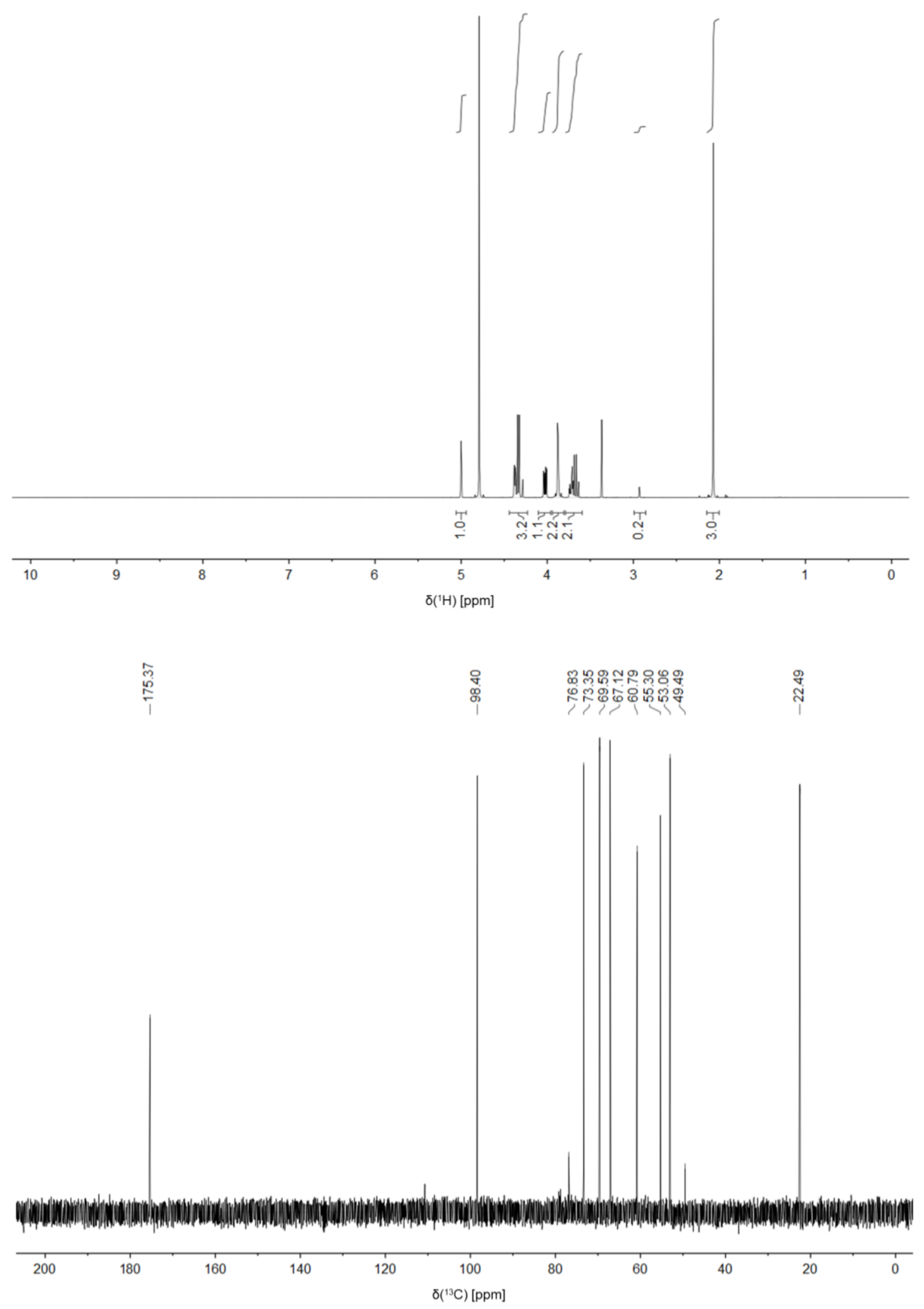

368

369 
371

372

373

374

375

376

377

378

379

380

381

382

383

384

385

386

387

388

389

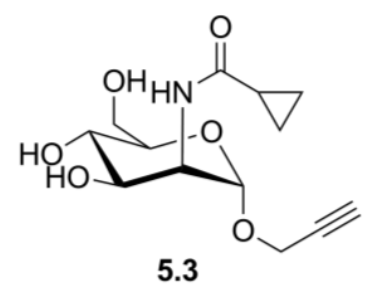

4 (48 mg, $220 \mu \mathrm{mol})$ was dissolved in pyridine $(5 \mathrm{ml})$ and cyclopropanecarbonyl chloride $(160 \mu \mathrm{l}, 1.8$ mmol, Acros Organics) was added at $0^{\circ} \mathrm{C}$. The mixture was allowed to heat up to room temperature and progress of the reaction was monitored by analytical TLC (3\% MeOH in DCM). After $7 \mathrm{~h} \mathrm{EtOH}$ containing 33\% methylamine $(10 \mathrm{ml})$ was added and the mixture was stirred overnight at room temperature. Solvents were evaporated in vacuo and the residue was purified via column chromatography (gradient: hexane, hexane:DCM (1:1), DCM and elution with $\mathrm{ml}$ 1\% MeOH in DCM) to yield $\mathbf{5 . 3}$ (27 mg, $95 \mu \mathrm{mol}, 43 \%)$ as a yellow solid.

${ }^{1} \mathrm{H}$ NMR (400.0 MHz, $\left.\mathrm{D}_{2} \mathrm{O}\right): \delta=5.00 \mathrm{ppm}, \mathrm{d}, 1 \mathrm{H}, \mathrm{J}=1.3 \mathrm{~Hz}(\mathrm{H1}) ; \delta=4.40 \mathrm{ppm}$, dd, $1 \mathrm{H}, \mathrm{J}=1.4,4.8$ $\mathrm{Hz}(\mathrm{H} 2) ; \delta=3.34 \mathrm{ppm}, \mathrm{m}, 2 \mathrm{H}(\mathrm{OCH} 2 \mathrm{CCH}) ; \delta=4.02 \mathrm{ppm}, \mathrm{m}, 1 \mathrm{H}(\mathrm{H} 3) ; \delta=3.89 \mathrm{ppm}, \mathrm{m}, 2 \mathrm{H}$ $(\mathrm{H} 6 \mathrm{a} / \mathrm{b}) ; \delta=3.72, \mathrm{~m}, 2 \mathrm{H}(\mathrm{H} 4, \mathrm{H} 5) ; \delta=2.93 \mathrm{ppm}, \mathrm{t}, \mathrm{J}=2.3 \mathrm{~Hz}\left(\mathrm{OCH}_{2} \mathrm{CCH}\right) ; \delta=1.73 \mathrm{ppm}, \mathrm{m}, 1 \mathrm{H}$ $\left(\mathrm{NHCOCHC}_{2} \mathrm{H}_{4}\right) ; \delta=0.89 \mathrm{ppm}, \mathrm{m}, 4 \mathrm{H}\left(\mathrm{NHCOCHC}_{2} H_{4}\right)$.

${ }^{13} \mathrm{C} \mathrm{NMR}\left(100.6 \mathrm{MHz}, \mathrm{D}_{2} \mathrm{O}\right): \delta=178.4 \mathrm{ppm}, 1 \mathrm{C}\left(\mathrm{NHCOCHC}_{2} \mathrm{H}_{4}\right) ; \delta=98.3 \mathrm{ppm}, 1 \mathrm{C}(\mathrm{C} 1) ; \delta=79.1$ ppm, $1 \mathrm{C}\left(\mathrm{OCH}_{2} \mathrm{CCH}\right) ; \delta=76.7 \mathrm{ppm}, 1 \mathrm{C}\left(\mathrm{OCH}_{2} \mathrm{CCH}\right) ; \delta=73.2 \mathrm{ppm}, 1 \mathrm{C}(\mathrm{C} 5) ; \delta=69.5,1 \mathrm{C}(\mathrm{C} 3) ; \delta$ $=67.0 \mathrm{ppm}, 1 \mathrm{C}(\mathrm{C} 4) ; \delta=60.7 \mathrm{ppm}, 1 \mathrm{C}(\mathrm{C} 6) ; \delta=55.2,1 \mathrm{C}\left(\mathrm{OCH}_{2} \mathrm{CCH}\right) ; \delta=53.0 \mathrm{ppm}, 1 \mathrm{C}(\mathrm{C} 2) ; \delta=$ $14.4 \mathrm{ppm}, 1 \mathrm{C}\left(\mathrm{NHCOCHC}_{2} \mathrm{H}_{4}\right) ; \delta=7.7 \mathrm{ppm}, 1 \mathrm{C}\left(\mathrm{NHCOCHC}_{2} \mathrm{H}_{4}\right) ; \delta=7.6 \mathrm{ppm}, 1 \mathrm{C}$ $\left(\mathrm{NHCOCHC}_{2} \mathrm{H}_{4}\right)$.

$\mathrm{R}_{\mathrm{f}}=0.51$ with $3 \% \mathrm{MeOH}$ in DCM

HR ESI-MS for $\mathrm{C}_{13} \mathrm{H}_{19} \mathrm{NO}_{6}: \mathrm{m} \cdot \mathrm{z}^{-1}\left(\mathrm{M}+\mathrm{Na}^{+}\right)_{\text {calc }}=308.111 ; \mathrm{m} \cdot \mathrm{z}^{-1}\left(\mathrm{M}+\mathrm{Na}^{+}\right)_{\text {obs }}=308.111$. 


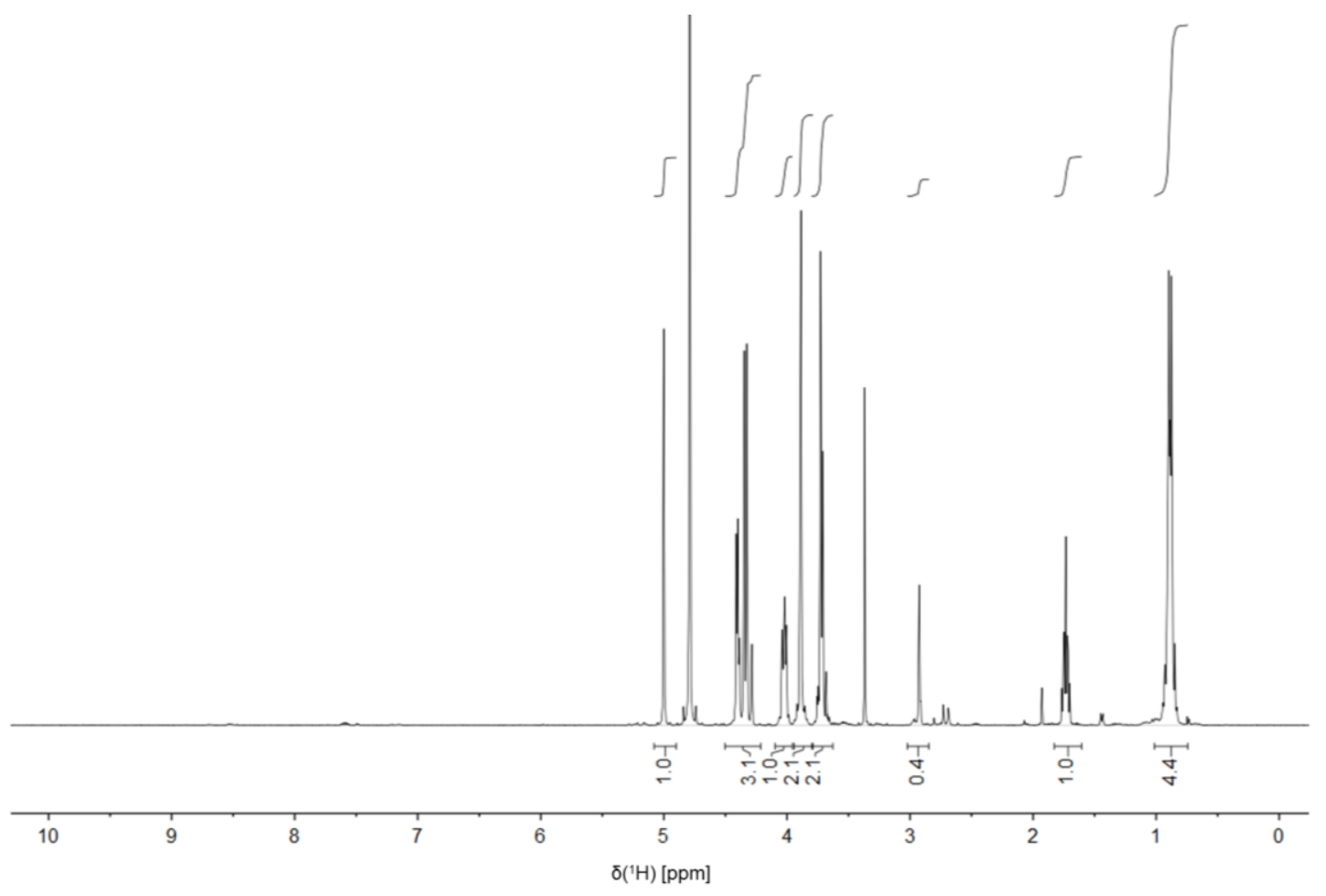

390
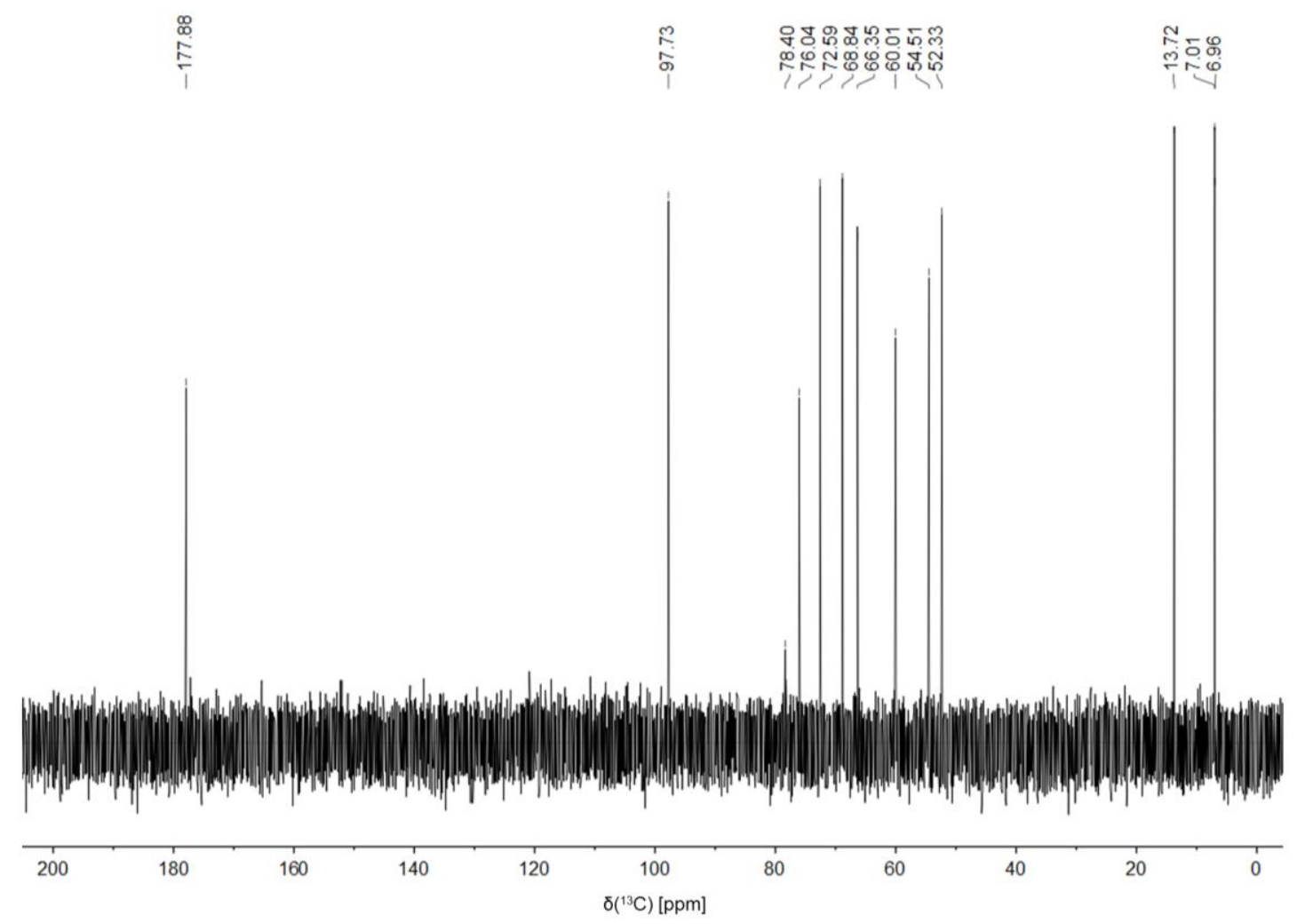

391

392 
394

395

396

397

398

399

400

401

402

403

404

405

406

407

408

409

410

411

412

413

414

415

416

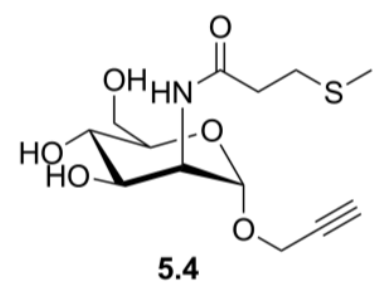

$4(3.03 \mathrm{mg}, 150 \mu \mathrm{mol})$ was dissolved in pyridine $(760 \mu \mathrm{l})$ and 3-(methylthio)propanoyl chloride (21 $\mu \mathrm{l}, 170 \mu \mathrm{mol}, \mathrm{TCI})$ was added at $0^{\circ} \mathrm{C}$. The reaction mixture was allowed to heat up to room temperature and after $4 \mathrm{~h}$ more 3-(methylthio)propanoyl chloride (21 $\mu \mathrm{l}, 170 \mu \mathrm{mol}$, TCI) was added. Progress of the reaction was monitored by analytical TLC (10\% MeOH in DCM) and after $7 \mathrm{~h} \mathrm{EtOH}$ containing $33 \%$ methylamine $(5 \mathrm{ml})$ was added at room temperature. The mixture was stirred overnight, solvents were evaporated in vacuo and the residue was purified via column chromatography (gradient: hexane, DCM, $1 \% \mathrm{MeOH}$ in DCM, 5\% MeOH in DCM and elution with $10 \% \mathrm{MeOH}$ in DCM). Residual impurities were removed via reversed-phase column chromatography (gradient: $\mathrm{H}_{2} \mathrm{O}$, $1 \% \mathrm{MeOH}$ in $\mathrm{H}_{2} \mathrm{O}$ and elution with $5 \% \mathrm{MeOH}$ in $\mathrm{H}_{2} \mathrm{O}$ ) to yield $\mathbf{5 . 4}(4.8 \mathrm{mg}, 15 \mu \mathrm{mol}, 10 \%)$ as a white solid.

${ }^{1} \mathrm{H}$ NMR (400.0 MHz, MeOD): $\delta=4.90$ ppm, s, $1 \mathrm{H}(\mathrm{H} 1) ; \delta=4.33$ ppm, d, $1 \mathrm{H}, \mathrm{J}=4.6 \mathrm{~Hz}(\mathrm{H} 2) ; \delta=$ 4.26 ppm, m, $2 \mathrm{H}(\mathrm{OCH} 2 \mathrm{CCH}) ; \delta=3.91 \mathrm{ppm}$, dd, J = 4.7, 9.1 Hz, $1 \mathrm{H}(\mathrm{H} 3) ; \delta=3.81 \mathrm{ppm}, \mathrm{d}, 2 \mathrm{H}, \mathrm{J}=$ $3.2 \mathrm{~Hz}(\mathrm{H6a} / \mathrm{b}) ; \delta=3.59, \mathrm{~m}, 1 \mathrm{H}(\mathrm{H} 4) ; \delta=3.54, \mathrm{dt}, 1 \mathrm{H}, \mathrm{J}=3.1,10.0 \mathrm{~Hz}(\mathrm{H} 5) ; \delta=2.87 \mathrm{ppm}, \mathrm{t}, \mathrm{J}=2.2$ $\mathrm{Hz}\left(\mathrm{OCH}_{2} \mathrm{CCH}\right) ; \delta=2.75 \mathrm{ppm}, \mathrm{m}, 2 \mathrm{H}\left(\mathrm{NHCOCH}_{2} \mathrm{CH}_{2} \mathrm{SCH}_{3}\right) ; \delta=2.58 \mathrm{ppm}, \mathrm{m}, 2 \mathrm{H}$ $\left(\mathrm{NHCOCH}_{2} \mathrm{CH}_{2} \mathrm{SCH}_{3}\right) ; \delta=2.12 \mathrm{ppm}$, s, $3 \mathrm{H}\left(\mathrm{NHCOCH}_{2} \mathrm{CH}_{2} \mathrm{SCH}_{3}\right)$.

${ }^{13} \mathrm{C} \mathrm{NMR}(100.6 \mathrm{MHz}, \mathrm{MeOD}): \delta=175.0 \mathrm{ppm}, 1 \mathrm{C}\left(\mathrm{NHCOCH}_{2} \mathrm{CH}_{2} \mathrm{SCH}_{3}\right) ; \delta=99.1 \mathrm{ppm}, 1 \mathrm{C}(\mathrm{C} 1) ; \delta$ $=79.8 \mathrm{ppm}, 1 \mathrm{C}\left(\mathrm{OCH}_{2} \mathrm{CCH}\right) ; \delta=76.2 \mathrm{ppm}, 1 \mathrm{C}\left(\mathrm{OCH}_{2} \mathrm{CCH}\right) ; \delta=74.6 \mathrm{ppm}, 1 \mathrm{C}(\mathrm{C} 5) ; \delta=70.7,1 \mathrm{C}$ $(\mathrm{C} 3) ; \delta=68.2 \mathrm{ppm}, 1 \mathrm{C}(\mathrm{C} 4) ; \delta=62.1 \mathrm{ppm}, 1 \mathrm{C}(\mathrm{C} 6) ; \delta=55.1,1 \mathrm{C}\left(\mathrm{OCH}_{2} \mathrm{CCH}\right) ; \delta=54.1 \mathrm{ppm}, 1 \mathrm{C}$ (C2); $\delta=36.8$ ppm, $1 \mathrm{C}\left(\mathrm{NHCOCH}_{2} \mathrm{CH}_{2} \mathrm{SCH}_{3}\right) ; \delta=30.8 \mathrm{ppm}, 1 \mathrm{C}\left(\mathrm{NHCOCH}_{2} \mathrm{CH}_{2} \mathrm{SCH}_{3}\right) ; \delta=15.3$ ppm, $1 \mathrm{C}\left(\mathrm{NHCOCH}_{2} \mathrm{CH}_{2} \mathrm{SCH}_{3}\right)$.

$\mathrm{R}_{\mathrm{f}}=0.18$ with $10 \% \mathrm{MeOH}$ in DCM.

HR ESI-MS for $\mathrm{C}_{13} \mathrm{H}_{21} \mathrm{NO}_{6} \mathrm{~S}: \mathrm{m} \cdot \mathrm{z}^{-1}\left(\mathrm{M}+\mathrm{Na}^{+}\right)_{\text {calc }}=342.099 ; \mathrm{m} \cdot \mathrm{z}^{-1}\left(\mathrm{M}+\mathrm{Na}^{+}\right)_{\text {obs }}=342.103$. 


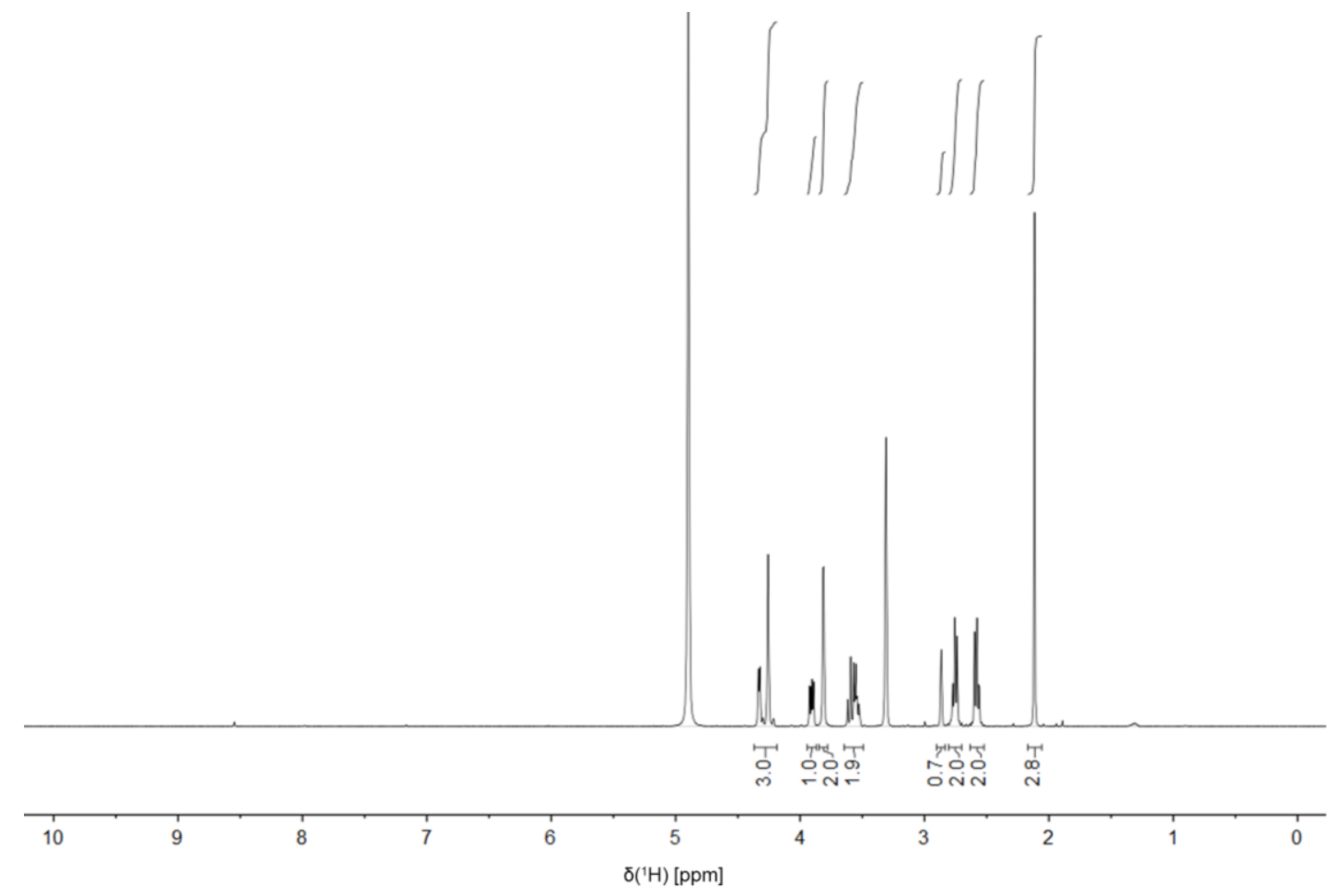

417

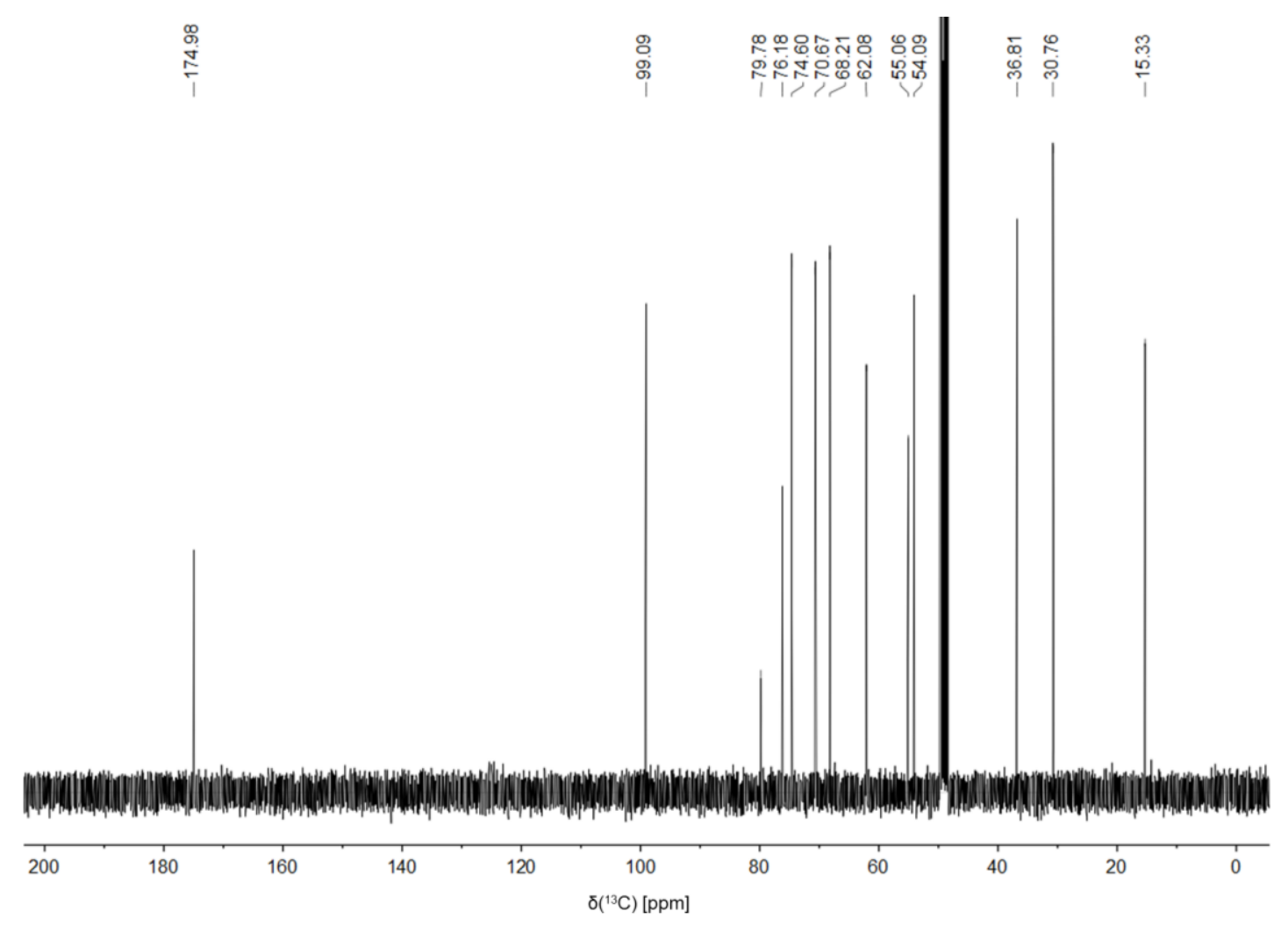

418

419 


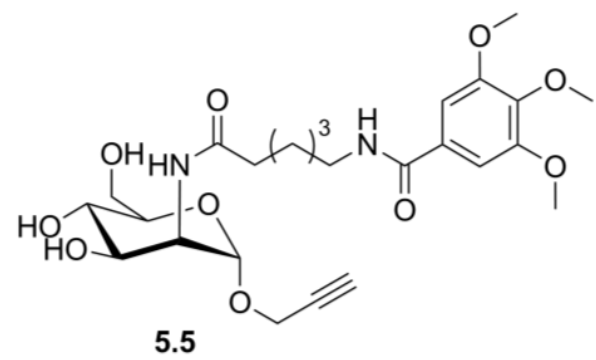

6-(3,4,5-Trimethoxybenzamido)hexanoic acid (90 mg, $0.28 \mathrm{mmol}$, Vitas-M Laboratory) was dissolved in DMF (700 $\mu$ l). Subsequently, PyBOP $(150 \mathrm{mg}, 0.28 \mathrm{mmol})$ and DIPEA (100 $\mu 1,0.55 \mathrm{mmol})$ were added and the mixture was stirred for $10 \mathrm{~min}$ at room temperature. $4(30 \mathrm{mg}, 0.14 \mathrm{mmol})$ was added and the mixture was stirred overnight at room temperature. Progress of the reaction was monitored by analytical TLC (10\% $\mathrm{MeOH}$ in DCM). The reaction was quenched with $\mathrm{MeOH}(1 \mathrm{ml})$ and after addition of $1 \mathrm{M} \mathrm{NaOH}(830 \mu 1,0.83 \mathrm{mmol})$ solvents were removed in vacuo. The residue was purified via reversed-phase column chromatography (gradient: $\mathrm{H}_{2} \mathrm{O}, 10 \% \mathrm{MeOH}$ in $\mathrm{H}_{2} \mathrm{O}, 20 \% \mathrm{MeOH}$ in $\mathrm{H}_{2} \mathrm{O}$, $30 \% \mathrm{MeOH}$ in $\mathrm{H}_{2} \mathrm{O}$ and elution with $40 \% \mathrm{MeOH}$ in $\mathrm{H}_{2} \mathrm{O}$ ) to yield 5.5 (51 mg, $97 \mu$ mol, $70 \%$ yield) as a white solid.

$432{ }^{1} \mathrm{H}$ NMR (400.0 MHz, MeOD): $\delta=7.17$ ppm, s, $2 \mathrm{H}$ (aromatic H of 3',4',5'-trimethoxybenzamide); $\delta$ $433=4.85$ ppm, s, $1 \mathrm{H}(\mathrm{H} 1) ; \delta=4.31 \mathrm{ppm}, \mathrm{d}, 1 \mathrm{H}, \mathrm{J}=5.1 \mathrm{~Hz}(\mathrm{H} 2) ; \delta=4.23 \mathrm{ppm}, \mathrm{m}, 2 \mathrm{H}\left(\mathrm{OCH}_{2} \mathrm{CCH}\right) ; \delta$ $434=3.91$ ppm, m, $1 \mathrm{H}(\mathrm{H} 3) ; \delta=3.89$ ppm, s, $6 \mathrm{H}$ (two times $\mathrm{CH}_{3}$ of 3', 4', 5'-trimethoxybenzamide); $\delta=$ $4353.81 \mathrm{ppm}, \mathrm{m}, 2 \mathrm{H},(\mathrm{H6a} / \mathrm{b}) ; \delta=3.81 \mathrm{ppm}, \mathrm{s}, 3 \mathrm{H}\left(\mathrm{CH}_{3}\right.$ of 3',4',5'-trimethoxybenzamide); $\delta=3.60$ $436 \mathrm{ppm}, \mathrm{m}, 1 \mathrm{H}(\mathrm{H} 4) ; \delta=3.53 \mathrm{ppm}, \mathrm{dt}, 1 \mathrm{H}, \mathrm{J}=3.2,10.1 \mathrm{~Hz}(\mathrm{H} 5) ; \delta=3.38 \mathrm{ppm}, \mathrm{m}, 2 \mathrm{H}$ $437\left(\mathrm{NHCOCH}_{2} \mathrm{CH}_{2} \mathrm{CH}_{2} \mathrm{CH}_{2} \mathrm{CH}_{2} \mathrm{NHCO}\right) ; \delta=2.85 \mathrm{ppm}, \mathrm{t}, \mathrm{J}=2.4 \mathrm{~Hz}\left(\mathrm{OCH}_{2} \mathrm{CCH}\right) ; \delta=2.31 \mathrm{ppm}, \mathrm{m}, 2 \mathrm{H}$ $438\left(\mathrm{NHCOCH}_{2} \mathrm{CH}_{2} \mathrm{CH}_{2} \mathrm{CH}_{2} \mathrm{CH}_{2} \mathrm{NHCO}\right) ; \delta=1.67 \mathrm{ppm}, \mathrm{m}, 4 \mathrm{H}\left(\mathrm{NHCOCH}_{2} \mathrm{CH}_{2} \mathrm{CH}_{2} \mathrm{CH}_{2} \mathrm{CH}_{2} \mathrm{NHCO}\right) ; \delta=$ 4391.43 ppm, m, $2 \mathrm{H}\left(\mathrm{NHCOCH}_{2} \mathrm{CH}_{2} \mathrm{CH}_{2} \mathrm{CH}_{2} \mathrm{CH}_{2} \mathrm{NHCO}\right)$. 
${ }^{13} \mathrm{C}$ NMR (100.6 MHz, MeOD): $\delta=175.0 \mathrm{ppm}, 1 \mathrm{C}\left(\mathrm{NHCOCH}_{2} \mathrm{CH}_{2} \mathrm{CH}_{2} \mathrm{CH}_{2} \mathrm{CH}_{2} \mathrm{NHCO}\right) ; \delta=169.5$

442 ppm, 1C $\left(\mathrm{NHCOCH}_{2} \mathrm{CH}_{2} \mathrm{CH}_{2} \mathrm{CH}_{2} \mathrm{CH}_{2} \mathrm{NHCO}\right) ; \delta=154.4 \mathrm{ppm}, 2 \mathrm{C}$ (C3' and $\mathrm{C} 5^{\prime}$ of 3', 4', 5'-

443 trimethoxybenzamide); $\delta=141.9 \mathrm{ppm}, 1 \mathrm{C}$ (C4' of 3', 4',5'-trimethoxybenzamide); $\delta=131.1 \mathrm{ppm}, 1 \mathrm{C}$

$444(\mathrm{C} 1$ ' of 3',4',5'-trimethoxybenzamide); $\delta=105.9 \mathrm{ppm}, 2 \mathrm{C}$ (C2' and C6' of 3',4',5'-

445 trimethoxybenzamide); $\delta=99.2 \mathrm{ppm}, 1 \mathrm{C}(\mathrm{C} 1) ; \delta=79.80 \mathrm{ppm}, 1 \mathrm{C}\left(\mathrm{OCH}_{2} \mathrm{CCH}\right) ; \delta=76.2 \mathrm{ppm}, 1 \mathrm{C}$

$446\left(\mathrm{OCH}_{2} \mathrm{CCH}\right) ; \delta=74.6 \mathrm{ppm}, 1 \mathrm{C}(\mathrm{C} 5) ; \delta=70.69,1 \mathrm{C}(\mathrm{C} 3) ; \delta=68.2 \mathrm{ppm}, 1 \mathrm{C}(\mathrm{C} 4) ; \delta=62.1 \mathrm{ppm}, 1 \mathrm{C}$

447 (C6); $\delta=61.1 \mathrm{ppm}, 1 \mathrm{C}\left(\mathrm{CH}_{3}\right.$ of 3', ',',5'-trimethoxybenzamide); $\delta=56.7 \mathrm{ppm}, 2 \mathrm{C}$ (two times $\mathrm{CH}_{3}$ of

448 3',4',5'-trimethoxybenzamide); $\delta=55.1,1 \mathrm{C}\left(\mathrm{OCH}_{2} \mathrm{CCH}\right) ; \delta=54.0 \mathrm{ppm}, 1 \mathrm{C}(\mathrm{C} 2) ; \delta=41.0 \mathrm{ppm}, 1 \mathrm{C}$

$449\left(\mathrm{NHCOCH}_{2} \mathrm{CH}_{2} \mathrm{CH}_{2} \mathrm{CH}_{2} \mathrm{CH}_{2} \mathrm{NHCO}\right) ; \delta=36.7 \mathrm{ppm}, 1 \mathrm{C}\left(\mathrm{NHCOCH}_{2} \mathrm{CH}_{2} \mathrm{CH}_{2} \mathrm{CH}_{2} \mathrm{CH}_{2} \mathrm{NHCO}\right) ; \delta=$

45030.2 ppm, 1 C $\quad\left(\mathrm{NHCOCH}_{2} \mathrm{CH}_{2} \mathrm{CH}_{2} \mathrm{CH}_{2} \mathrm{CH}_{2} \mathrm{NHCO}\right) ; \quad \delta=27.6 \quad$ ppm, $1 \quad \mathrm{C}$

$451 \quad\left(\mathrm{NHCOCH}_{2} \mathrm{CH}_{2} \mathrm{CH}_{2} \mathrm{CH}_{2} \mathrm{CH}_{2} \mathrm{NHCO}\right) ; \delta=26.6 \mathrm{ppm}, 1 \mathrm{C}\left(\mathrm{NHCOCH}_{2} \mathrm{CH}_{2} \mathrm{CH}_{2} \mathrm{CH}_{2} \mathrm{CH}_{2} \mathrm{NHCO}\right)$.

$452 \mathrm{R}_{\mathrm{f}}=0.23$ with $10 \% \mathrm{MeOH}$ in DCM.

453 HR ESI-MS for $\mathrm{C}_{25} \mathrm{H}_{36} \mathrm{~N}_{2} \mathrm{O}_{10}: \mathrm{m} \cdot \mathrm{z}^{-1}\left(\mathrm{M}+\mathrm{Na}^{+}\right)_{\text {calc }}=547.227 ; \mathrm{m} \cdot \mathrm{z}^{-1}\left(\mathrm{M}+\mathrm{Na}^{+}\right)_{\text {obs }}=547.234$.

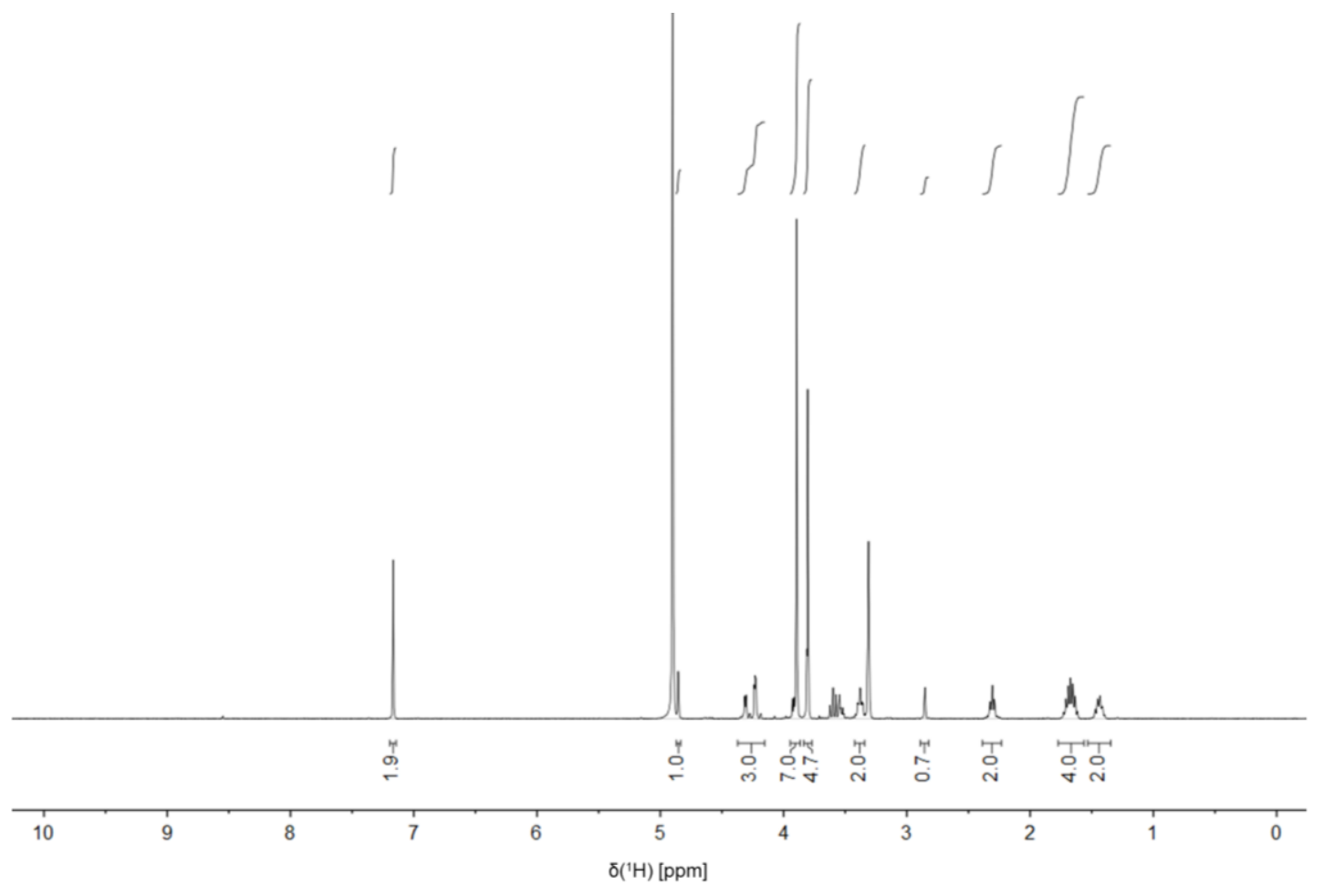




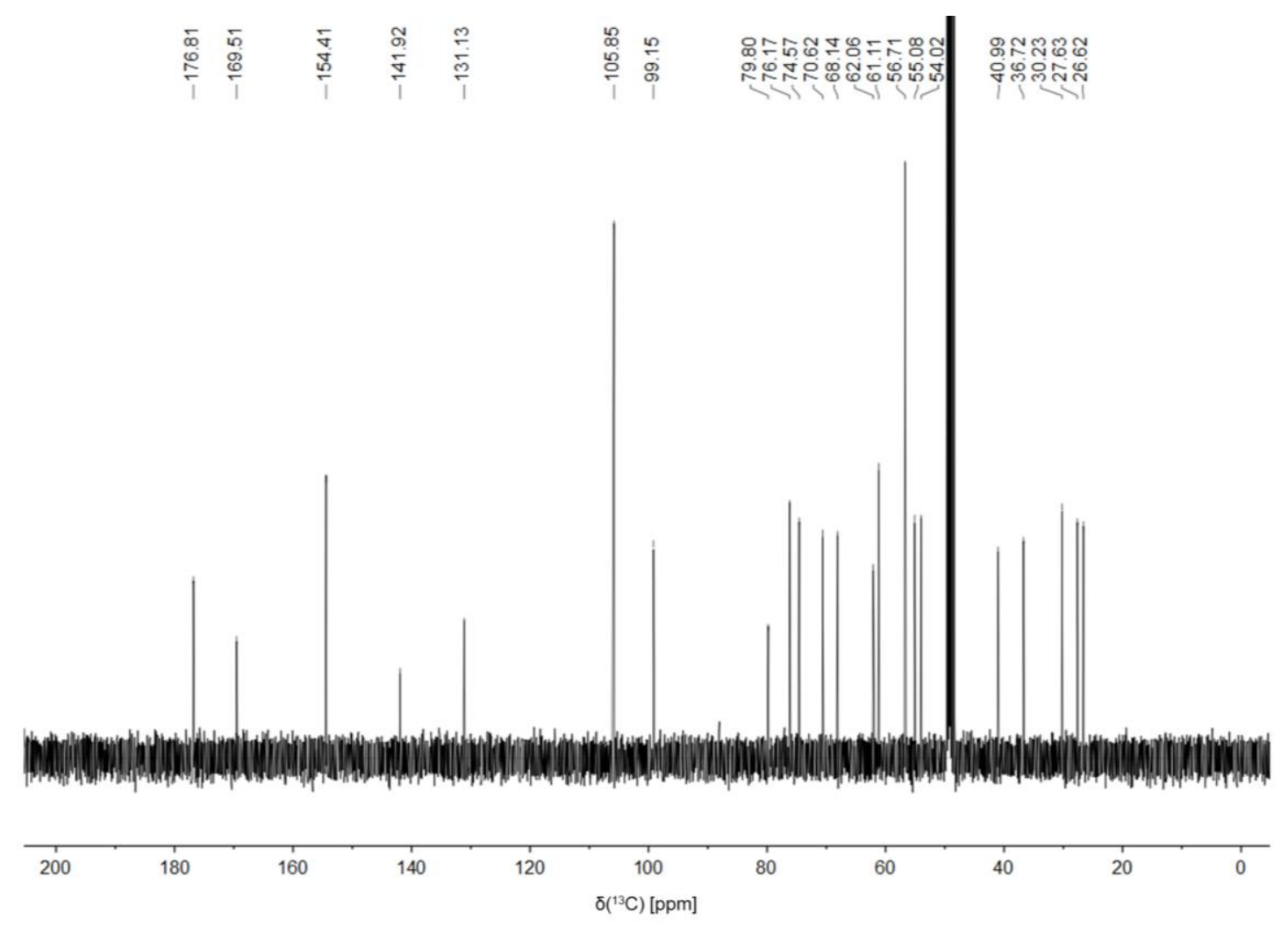

455

456 
458

459

460

461

462

463

464

465

466

467

468

469

470

471

472

473

474

475

476

477

478

479

480

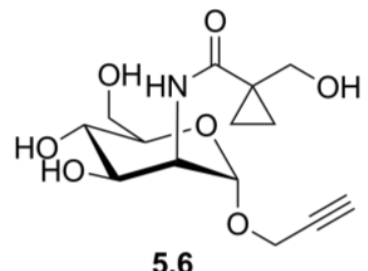

5.6

1-hydroxycyclopropanecarboxylic acid (37 mg, $0.32 \mathrm{mmol}$, ChemBridge) was dissolved in DMF (800 $\mu 1)$. Subsequently, PyBOP (170 mg, $0.32 \mathrm{mmol})$ and DIPEA (120 $\mu 1,0.65 \mathrm{mmol})$ were added and the mixture was stirred for $10 \mathrm{~min}$ at room temperature. $4(35 \mathrm{mg}, 0.16 \mathrm{mmol})$ was added, the mixture was stirred overnight at room temperature. Progress of the reaction was monitored by analytical TLC (15\% $\mathrm{MeOH}$ in DCM). The reaction was quenched with $\mathrm{MeOH}(1 \mathrm{ml})$ and after addition of $1 \mathrm{M} \mathrm{NaOH}$ $(1000 \mu \mathrm{l}, 1.0 \mathrm{mmol})$ solvents were removed in vacuo. The residue was purified via preparative HPLC (gradient: $\mathrm{H}_{2} \mathrm{O}$ for $10 \mathrm{~min}$, from 0 to $20 \%$ acetonitrile in $\mathrm{H}_{2} \mathrm{O}$ in $30 \mathrm{~min}$, from 20 to $50 \%$ acetonitrile in $\mathrm{H}_{2} \mathrm{O}$ in $10 \mathrm{~min}$, from 50 to $100 \%$ acetonitrile in $\mathrm{H}_{2} \mathrm{O}$ in $5 \mathrm{~min}$ and acetonitrile for $5 \mathrm{~min}$ at $3.2 \mathrm{ml} \cdot \mathrm{min}^{-}$ ${ }^{1}$ ) to yield $\mathbf{5 . 6}(16 \mathrm{mg}, 49 \mu \mathrm{mol}, 31 \%)$ as a white solid.

${ }^{1} \mathrm{H}$ NMR (400.0 MHz, MeOD): $\delta=4.98$ ppm, s, $1 \mathrm{H}(\mathrm{H} 1) ; \delta=4.25$ ppm, m, $2 \mathrm{H}(\mathrm{OCH} 2 \mathrm{CCH}) ; \delta=$ $4.23 \mathrm{ppm}, \mathrm{m}, 1 \mathrm{H}(\mathrm{H} 2) ; \delta=3.95 \mathrm{ppm}, \mathrm{dd}, 1 \mathrm{H}, \mathrm{J}=4.7,9.3 \mathrm{~Hz}(\mathrm{H} 3) ; \delta=3.85 \mathrm{ppm}$, dd, $1 \mathrm{H}, \mathrm{J}=1.9$,

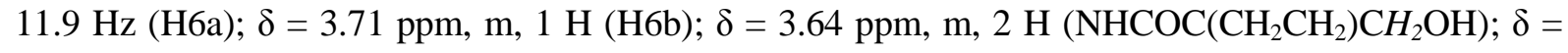
$3.55, \mathrm{~m}, 1 \mathrm{H}(\mathrm{H} 5) ; \delta=3.59, \mathrm{~m}, 1 \mathrm{H}(\mathrm{H} 4) ; \delta=2.87 \mathrm{ppm}, \mathrm{m}, 1 \mathrm{H}\left(\mathrm{OCH}_{2} \mathrm{CCH}\right) ; \delta=1.12 \mathrm{ppm}, \mathrm{m}, 2 \mathrm{H}$

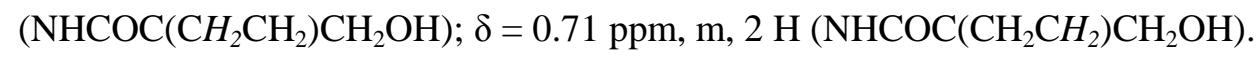

${ }^{13} \mathrm{C}$ NMR (100.6 MHz, MeOD): $\delta=177.0 \mathrm{ppm}, 1 \mathrm{C}\left(\mathrm{NHCOC}\left(\mathrm{CH}_{2} \mathrm{CH}_{2}\right) \mathrm{CH}_{2} \mathrm{OH}\right) ; \delta=98.5 \mathrm{ppm}, 1 \mathrm{C}$ $(\mathrm{C} 1) ; \delta=79.8 \mathrm{ppm}, 1 \mathrm{C}\left(\mathrm{OCH}_{2} \mathrm{CCH}\right) ; \delta=76.2 \mathrm{ppm}, 1 \mathrm{C}\left(\mathrm{OCH}_{2} \mathrm{CCH}\right) ; \delta=74.7 \mathrm{ppm}, 1 \mathrm{C}(\mathrm{C} 5) ; \delta=$

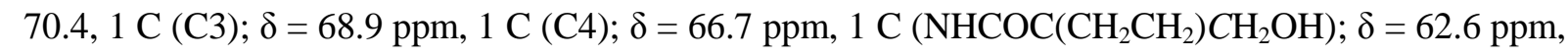
$1 \mathrm{C}(\mathrm{C} 6) ; \delta=55.1 \mathrm{ppm}, 1 \mathrm{C}\left(\mathrm{OCH}_{2} \mathrm{CCH}\right) ; \delta=54.4 \mathrm{ppm}, 1 \mathrm{C}(\mathrm{C} 2) ; \delta=26.4 \mathrm{ppm}, 1 \mathrm{C}$ $\left(\mathrm{NHCOC}\left(\mathrm{CH}_{2} \mathrm{CH}_{2}\right) \mathrm{CH}_{2} \mathrm{OH}\right) ; \delta=13.4 \mathrm{ppm}, 1 \mathrm{C}\left(\mathrm{NHCOC}\left(\mathrm{CH}_{2} \mathrm{CH}_{2}\right) \mathrm{CH}_{2} \mathrm{OH}\right) ; \delta=13.2 \mathrm{ppm}, 1 \mathrm{C}$ $\left(\mathrm{NHCOC}\left(\mathrm{CH}_{2} \mathrm{CH}_{2}\right) \mathrm{CH}_{2} \mathrm{OH}\right)$.

$\mathrm{R}_{\mathrm{f}}=0.33$ with $15 \% \mathrm{MeOH}$ in DCM.

HR ESI-MS for $\mathrm{C}_{14} \mathrm{H}_{21} \mathrm{NO}_{7}: \mathrm{m} \cdot \mathrm{z}^{-1}\left(\mathrm{M}+\mathrm{Na}^{+}\right)_{\text {calc }}=338.122 ; \mathrm{m} \cdot \mathrm{z}^{-1}\left(\mathrm{M}+\mathrm{Na}^{+}\right)_{\text {obs }}=338.121$. 


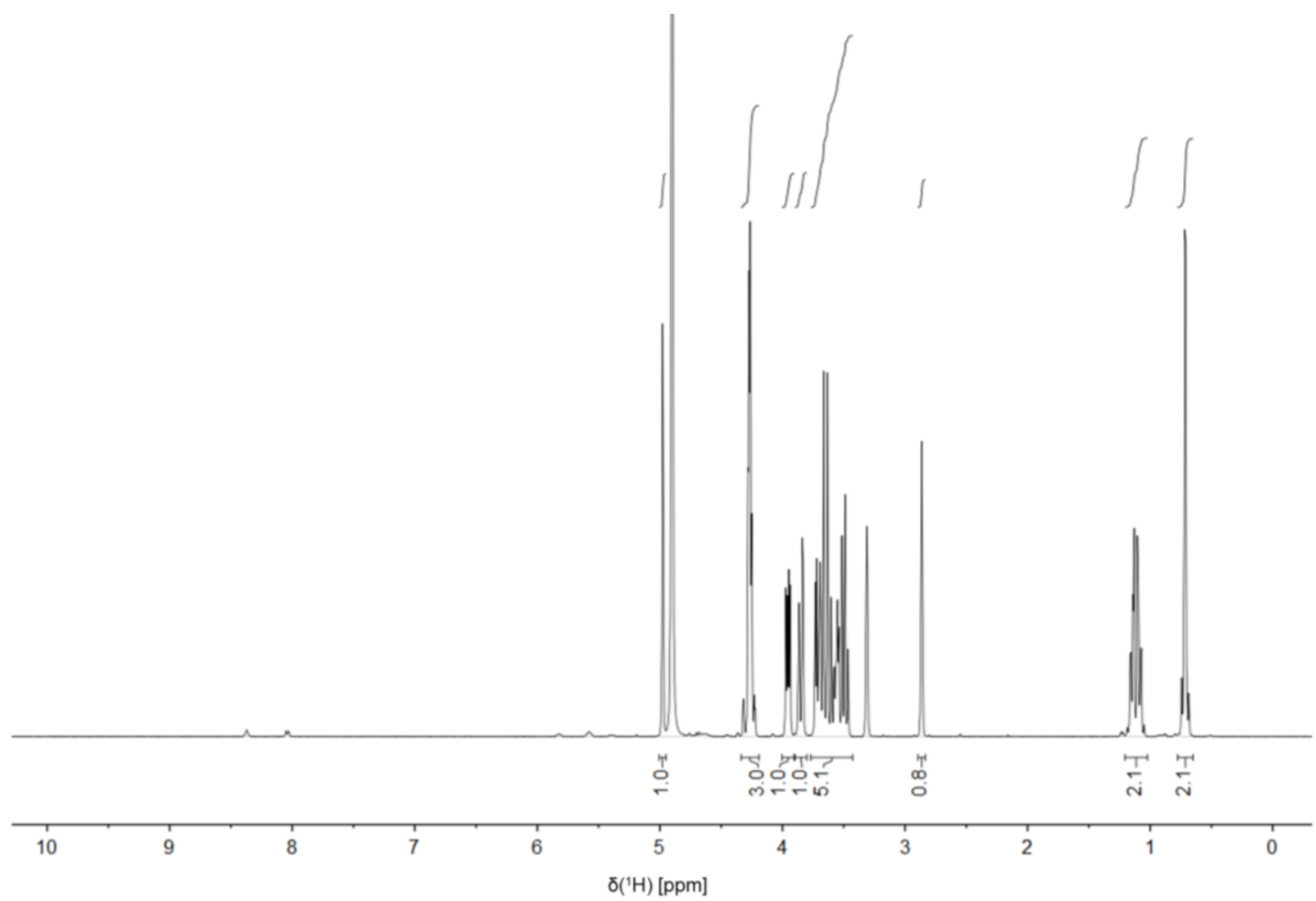

481

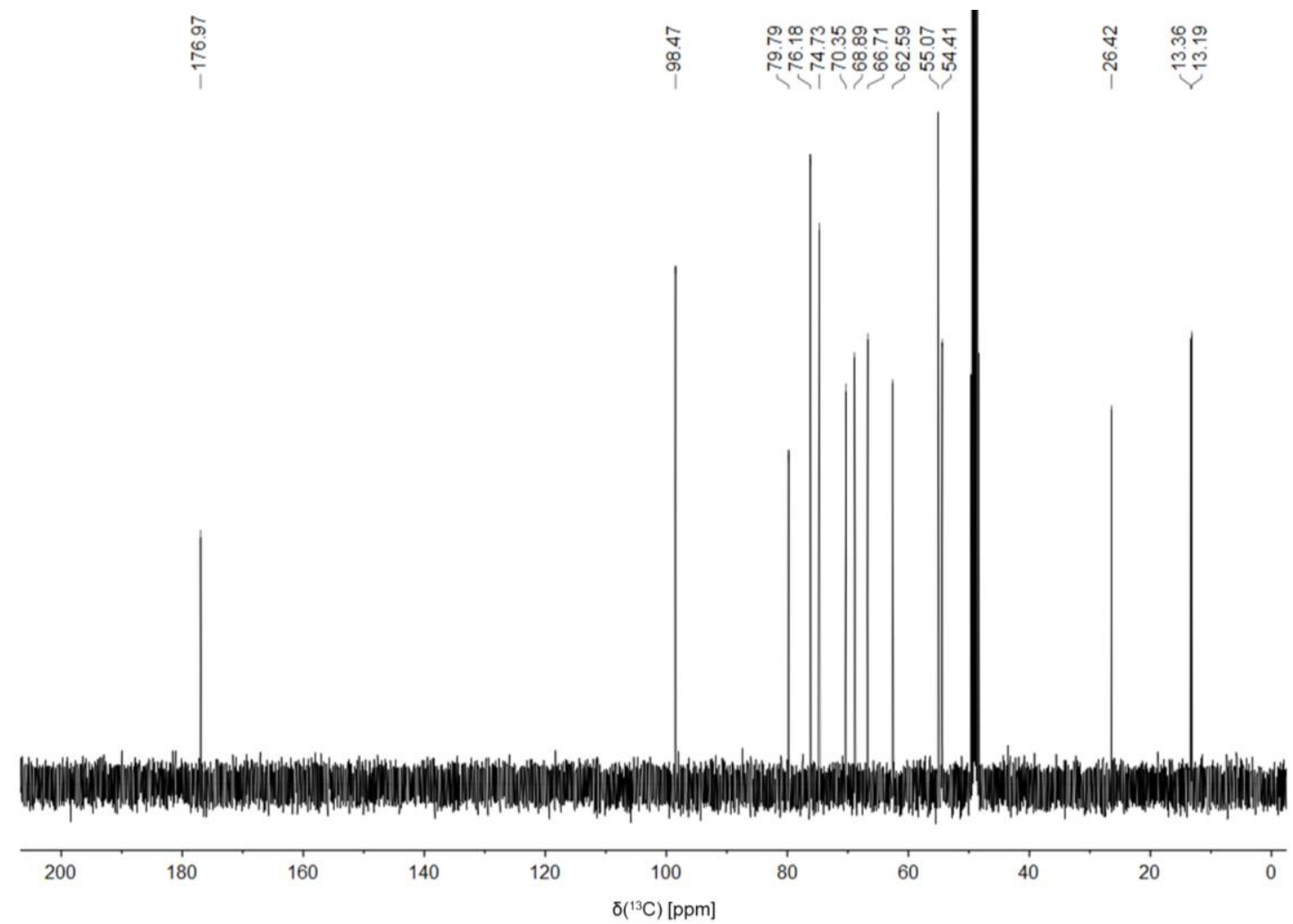

482

483 
485

486

487

488

489

490

491

492

493

494

495

496

497

498

499

500

501

502

503

504

505

506

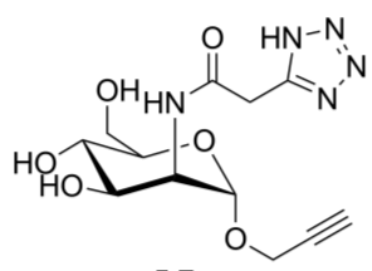

5.7

2-(tetrazolidin-5-yl)acetic acid (59 mg, $0.46 \mathrm{mmol}$, Santa Cruz Biotechnology) was dissolved in DMF (1.2 ml). Subsequently, PyBOP (240 mg, $0.46 \mathrm{mmol})$ and DIPEA (160 $\mu 1,0.92 \mathrm{mmol})$ were added and mixture was stirred for $10 \mathrm{~min}$ at room temperature. $4(50 \mathrm{mg}, 0.230 \mathrm{mmol}$ ) was added and the mixture was stirred overnight at room temperature. Progress of the reaction was monitored by analytical TLC (20\% $\mathrm{MeOH}$ in DCM). Following quenching with $\mathrm{MeOH}(1 \mathrm{ml})$, solvents were removed in vacuo. The residue was purified via reversed-phase column chromatography (gradient: elution with $\mathrm{H}_{2} \mathrm{O}$ ). Residual impurities were removed via preparative HPLC (gradient: $\mathrm{H}_{2} \mathrm{O}$ for 10 min, from 0 to $20 \%$ acetonitrile in $\mathrm{H}_{2} \mathrm{O}$ in $30 \mathrm{~min}$, from 20 to $50 \%$ acetonitrile in $\mathrm{H}_{2} \mathrm{O}$ in $10 \mathrm{~min}$, from 50 to $100 \%$ acetonitrile in $\mathrm{H}_{2} \mathrm{O}$ in $5 \mathrm{~min}$ and acetonitrile for $5 \mathrm{~min}$ at $3.2 \mathrm{ml} \cdot \mathrm{min}^{-1}$ ) to yield $\mathbf{5 . 7}$ as a white solid (16 mg, $49 \mu \mathrm{mol}, 21 \%$ ).

${ }^{1} \mathrm{H}$ NMR (400.0 MHz, MeOD): $\delta=8.11$ ppm, s, $1 \mathrm{H}\left(\mathrm{NHCOCH}_{2} \mathrm{~N}_{4} \mathrm{H}\right) ; \delta=4.94$ ppm, s, $1 \mathrm{H}(\mathrm{H} 1) ; \delta=$ $4.25 \mathrm{ppm}, \mathrm{d}, 1 \mathrm{H}, \mathrm{J}=4.7 \mathrm{~Hz}(\mathrm{H} 2) ; \delta=4.27 \mathrm{ppm}, \mathrm{m}, 2 \mathrm{H}(\mathrm{OCH} 2 \mathrm{CCH}) ; \delta=4.03 \mathrm{ppm}, \mathrm{m}, 2 \mathrm{H}$ $\left(\mathrm{NHCOCH}_{2} \mathrm{~N}_{4} \mathrm{H}\right) ; \delta=3.93 \mathrm{ppm}, \mathrm{dd}, 1 \mathrm{H}, \mathrm{J}=4.7,8.9 \mathrm{~Hz}(\mathrm{H} 3) ; \delta=3.83$ ppm, m, $2 \mathrm{H}(\mathrm{H} 6 \mathrm{a} / \mathrm{b}) ; \delta=3.61$ ppm, m, $1 \mathrm{H}(\mathrm{H} 4) ; \delta=3.58$ ppm, m, $1 \mathrm{H}(\mathrm{H} 5) ; \delta=2.87$ ppm, m, $1 \mathrm{H}\left(\mathrm{OCH}_{2} \mathrm{CC} H\right)$.

${ }^{13} \mathrm{C}$ NMR (100.6 MHz, MeOD): $\delta=169.5$ ppm, $1 \mathrm{C}\left(\mathrm{NHCOCH}_{2} \mathrm{~N}_{4} \mathrm{H}\right) ; \delta=98.9 \mathrm{ppm}, 1 \mathrm{C}(\mathrm{C} 1) ; \delta=$ 79.7 ppm, $1 \mathrm{C}\left(\mathrm{OCH}_{2} \mathrm{CCH}\right) ; \delta=76.2 \mathrm{ppm}, 1 \mathrm{C}\left(\mathrm{OCH}_{2} \mathrm{CCH}\right) ; \delta=74.7 \mathrm{ppm}, 1 \mathrm{C}(\mathrm{C} 5) ; \delta=70.7 \mathrm{ppm}, 1$ $\mathrm{C}(\mathrm{C} 3) ; \delta=68.3 \mathrm{ppm}, 1 \mathrm{C}(\mathrm{C} 4) ; \delta=62.2 \mathrm{ppm}, 1 \mathrm{C}(\mathrm{C} 6) ; \delta=55.1,1 \mathrm{C}\left(\mathrm{OCH}_{2} \mathrm{CCH}\right) ; \delta=54.4 \mathrm{ppm}, 1$ $\mathrm{C}(\mathrm{C} 2) ; \delta=31.0 \mathrm{ppm}, 1 \mathrm{C}\left(\mathrm{NHCOCH}_{2} \mathrm{~N}_{4} \mathrm{H}\right)$.

$\mathrm{R}_{\mathrm{f}}=0.06$ with $20 \% \mathrm{MeOH}$ in DCM.

HR ESI-MS for $\mathrm{C}_{12} \mathrm{H}_{17} \mathrm{~N}_{5} \mathrm{O}_{6}: \mathrm{m} \cdot \mathrm{z}^{-1}\left(\mathrm{M}+\mathrm{Na}^{+}\right)_{\text {calc }}=350.108 ; \mathrm{m} \cdot \mathrm{z}^{-1}\left(\mathrm{M}+\mathrm{Na}^{+}\right)_{\mathrm{obs}}=350.109$. 


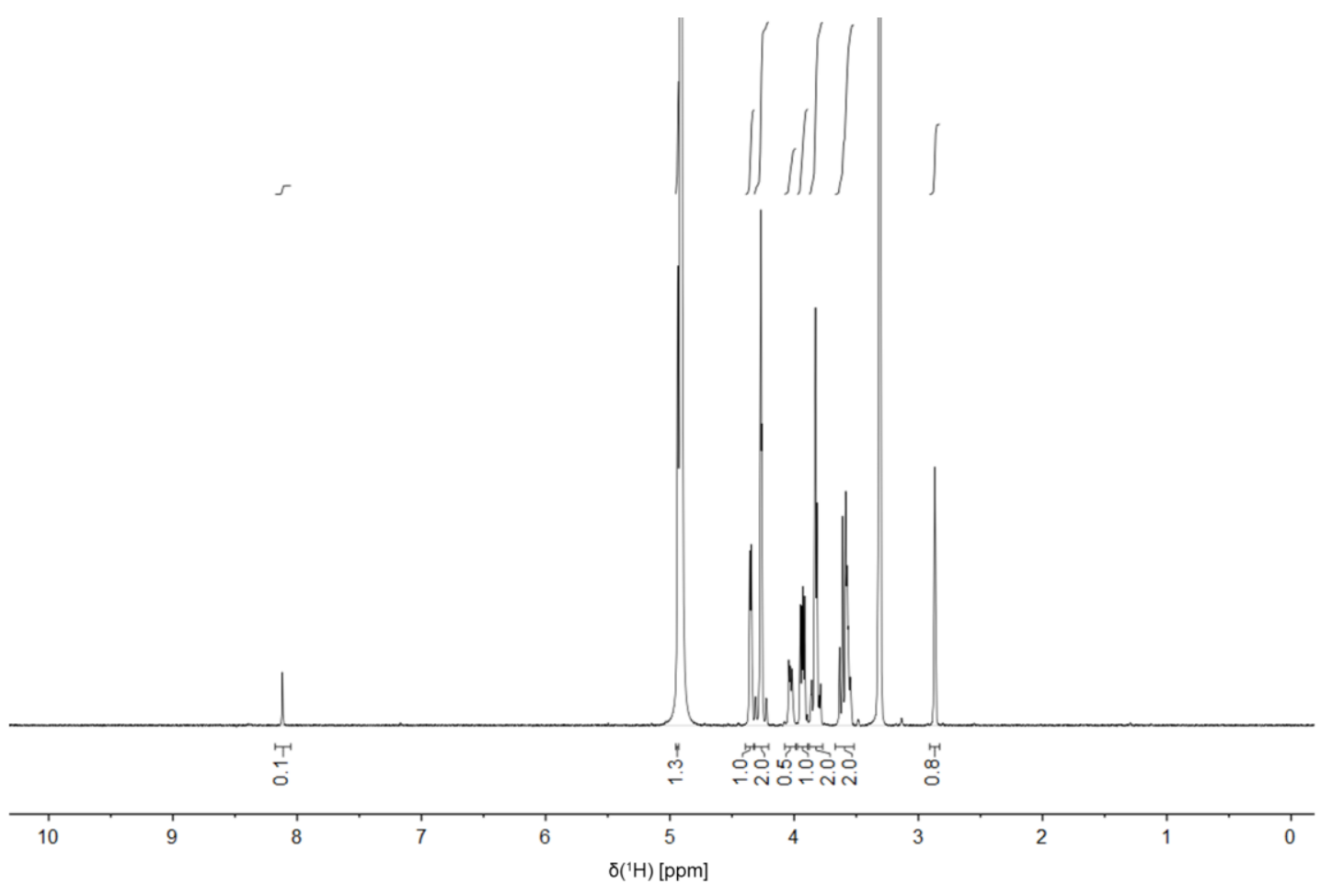

507

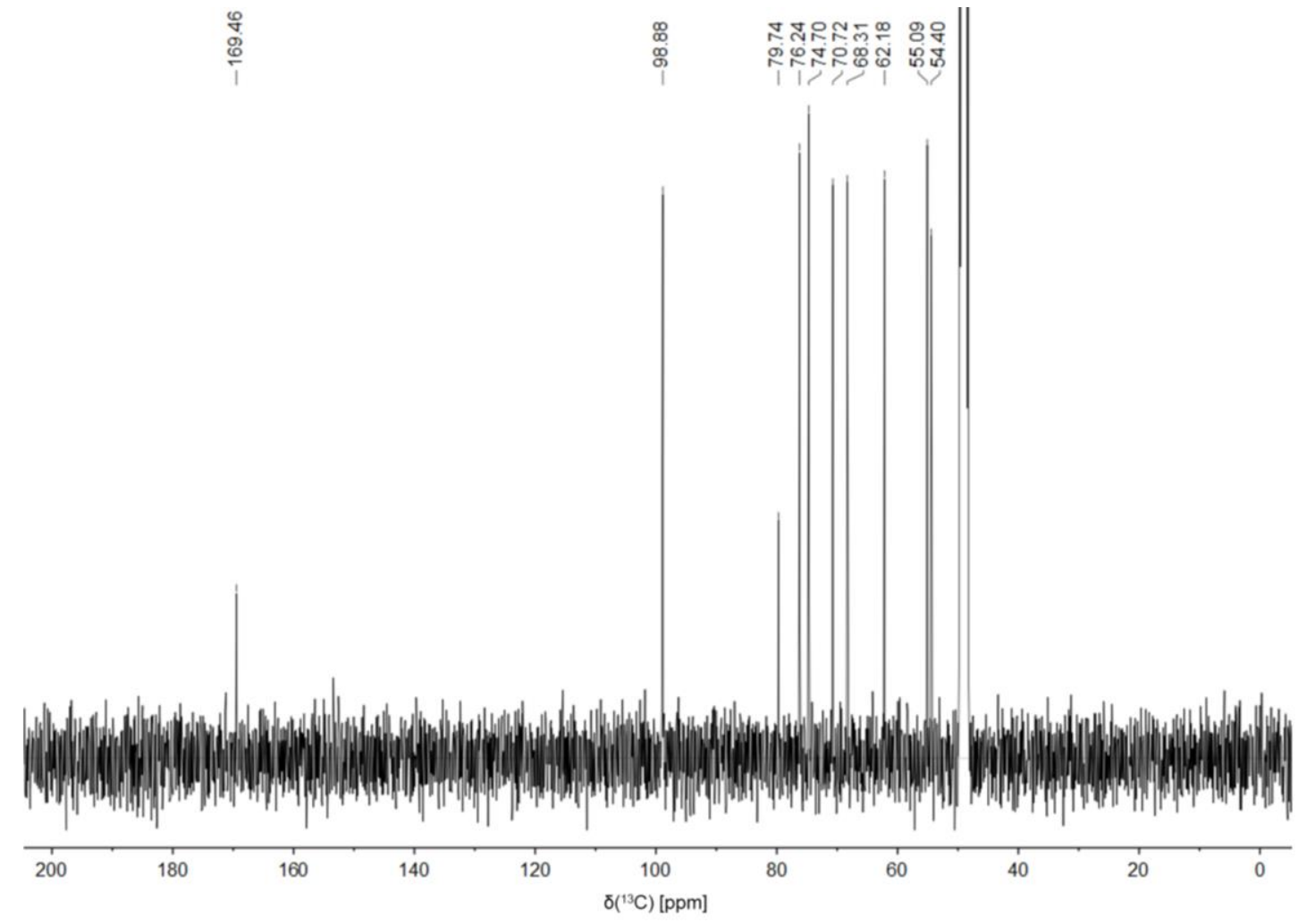




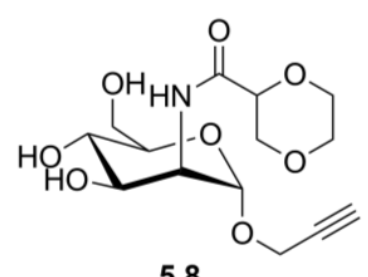

5.8

1,4-dioxane-2-carboxylic acid (40 mg, $306 \mu \mathrm{mol}$, Santa Cruz Biotechnology) was dissolved in DMF (760 $\mu \mathrm{l})$. Subsequently PyBOP $(158 \mathrm{mg}, 306 \mu \mathrm{mol})$ and DIPEA $(110 \mu \mathrm{l}, 612 \mu \mathrm{mol})$ were added and the mixture was stirred for $10 \mathrm{~min}$ at room temperature. $4(33 \mathrm{mg}, 152 \mu \mathrm{mol})$ was added and the mixture was stirred overnight at room temperature. Progress of the reaction was monitored by analytical TLC (10\% MeOH in DCM). Solvents were removed in vacuo and the residue was purified via column chromatography (gradient: hexane, DCM, $5 \% \mathrm{MeOH}$ in DCM and elution with $10 \%$ $\mathrm{MeOH}$ in DCM). Residual impurities were removed via revers-phase column chromatography (gradient: $\mathrm{H}_{2} \mathrm{O}, 5 \% \mathrm{MeOH}$ in $\mathrm{H}_{2} \mathrm{O}$ and elution at $10 \% \mathrm{MeOH}$ in $\mathrm{H}_{2} \mathrm{O}$ ) to yield $\mathbf{5 . 8}$ as a white solid (20 $\mathrm{mg}, 60 \mu \mathrm{mol}, 40 \%)$.

${ }^{1} \mathrm{H}$ NMR $(400 \mathrm{MHz}, \mathrm{MeOD}: \delta=4.95 \mathrm{ppm}, \mathrm{dd}, 1 \mathrm{H}, \mathrm{J}=1.0,8.4 \mathrm{~Hz}(\mathrm{H} 1) ; \delta=4.27 \mathrm{ppm}, \mathrm{m}, 2 \mathrm{H}$ $\left(\mathrm{OCH}_{2} \mathrm{CCH}\right) ; \delta=4.25$ ppm, m, $1 \mathrm{H}(\mathrm{H} 2) ; \delta=4.15$ ppm, m, $1 \mathrm{H}\left(\mathrm{NHCOCHCH}_{2} \mathrm{OCH}_{2} \mathrm{CH}_{2} \mathrm{O}\right) ; \delta=3.99$ ppm, m, $1 \mathrm{H}\left(\mathrm{NHCOCHCH}_{2} \mathrm{OCH}_{2} \mathrm{CH}_{2} \mathrm{O}\right) ; \delta=3.94 \mathrm{ppm}, \mathrm{m}, 1 \mathrm{H}(\mathrm{H} 3) ; \delta=3.90 \mathrm{ppm}, \mathrm{m}, 1 \mathrm{H}$ $\left(\mathrm{NHCOCHCH}_{2} \mathrm{OCH}_{2} \mathrm{CH}_{2} \mathrm{O}\right) ; \delta=3.79 \mathrm{ppm}, \mathrm{m}, 3 \mathrm{H}\left(\mathrm{H} 6 \mathrm{a} / \mathrm{b}, \mathrm{NHCOCHCH}_{2} \mathrm{OCH}_{2} \mathrm{CH}_{2} \mathrm{O}\right) ; \delta=3.72$ ppm, m, $1 \mathrm{H}\left(\mathrm{NHCOCHCH}_{2} \mathrm{OCH}_{2} \mathrm{CH}_{2} \mathrm{O}\right) ; \delta=3.62 \mathrm{ppm}, \mathrm{m}, 1 \mathrm{H}\left(\mathrm{NHCOCHCH}_{2} \mathrm{OCH}_{2} \mathrm{CH}_{2} \mathrm{O}\right) ; \delta=3.54$ ppm, m, $1 \mathrm{H}(\mathrm{H} 5) ; \delta=3.52 \mathrm{ppm}, \mathrm{m}, 1 \mathrm{H}(\mathrm{H} 4) ; \delta=3.47 \mathrm{ppm}, \mathrm{m}, 1 \mathrm{H}\left(\mathrm{NHCOCHCH}_{2} \mathrm{OCH}_{2} \mathrm{CH}_{2} \mathrm{O}\right) ; \delta=$ $2.87 \mathrm{ppm}, \mathrm{m}, 1 \mathrm{H}\left(\mathrm{OCH}_{2} \mathrm{CCH}\right)$

${ }^{13} \mathrm{C}$ NMR (100 MHz, MeOD): $\delta=171.5$ ppm, $1 \mathrm{C}\left(\mathrm{NHCOCHCH}_{2} \mathrm{OCH}_{2} \mathrm{CH}_{2} \mathrm{O}\right) ; \delta=98.8 \mathrm{ppm}, \mathrm{d}, 1 \mathrm{C}$, $\mathrm{J}=16.5 \mathrm{~Hz}(\mathrm{C} 1) ; \delta=79.7 \mathrm{ppm}, 1 \mathrm{C}\left(\mathrm{OCH}_{2} \mathrm{CCH}\right) ; \delta=76.3 \mathrm{ppm}, \mathrm{m}, 1 \mathrm{C}\left(\mathrm{NHCOCHCH}_{2} \mathrm{OCH}_{2} \mathrm{CH}_{2} \mathrm{O}\right)$; $\delta=76.2 \mathrm{ppm}, 1 \mathrm{C}\left(\mathrm{OCH}_{2} \mathrm{CCH}\right) ; \delta=74.6 \mathrm{ppm}, \mathrm{d}, 1 \mathrm{C}, \mathrm{J}=4.2 \mathrm{~Hz}(\mathrm{C} 5) ; \delta=70.3 \mathrm{ppm}, \mathrm{d}, \mathrm{J}=20.0 \mathrm{~Hz}$ $(\mathrm{C} 3) ; \delta=69.4 \mathrm{ppm}, \mathrm{d}, 1 \mathrm{C}, \mathrm{J}=8.9 \mathrm{~Hz}\left(\mathrm{NHCOCHCH}_{2} \mathrm{OCH}_{2} \mathrm{CH}_{2} \mathrm{O}\right) ; \delta=68.2 \mathrm{ppm}, 1 \mathrm{C}(\mathrm{C} 4) ; \delta=67.6$ ppm, d, $1 \mathrm{C}, \quad \mathrm{J}=3.9 \mathrm{~Hz}\left(\mathrm{NHCOCHCH}_{2} \mathrm{OCH}_{2} \mathrm{CH}_{2} \mathrm{O}\right) ; \delta=67.3 \mathrm{ppm}, \mathrm{d}, 1 \mathrm{C}, \mathrm{J}=1.6 \mathrm{~Hz}$ $\left(\mathrm{NHCOCHCH}_{2} \mathrm{OCH}_{2} \mathrm{CH}_{2} \mathrm{O}\right) ; \delta=62.0 \mathrm{ppm}, \mathrm{d}, 1 \mathrm{C}, \mathrm{J}=1.3 \mathrm{~Hz}(\mathrm{C} 6) ; \delta=55.2 \mathrm{ppm}, 1 \mathrm{C}\left(\mathrm{OCH}_{2} \mathrm{CCH}\right) ; \delta$ $=53.81 \mathrm{ppm}, \mathrm{d}, 1 \mathrm{C}, \mathrm{J}=4.9 \mathrm{~Hz}(\mathrm{C} 2)$

$\mathrm{R}_{\mathrm{f}}=0.19$ with $10 \%$ in $\mathrm{MeOH}$ in DCM.

HR ESI-MS for $\mathrm{C}_{14} \mathrm{H}_{21} \mathrm{NO}_{8}: \mathrm{m} \cdot \mathrm{z}^{-1}\left(\mathrm{M}+\mathrm{Na}^{+}\right)_{\text {calc }}=354.117 ; \mathrm{m} \cdot \mathrm{z}^{-1}\left(\mathrm{M}+\mathrm{Na}^{+}\right)_{\text {obs }}=354.114$. 


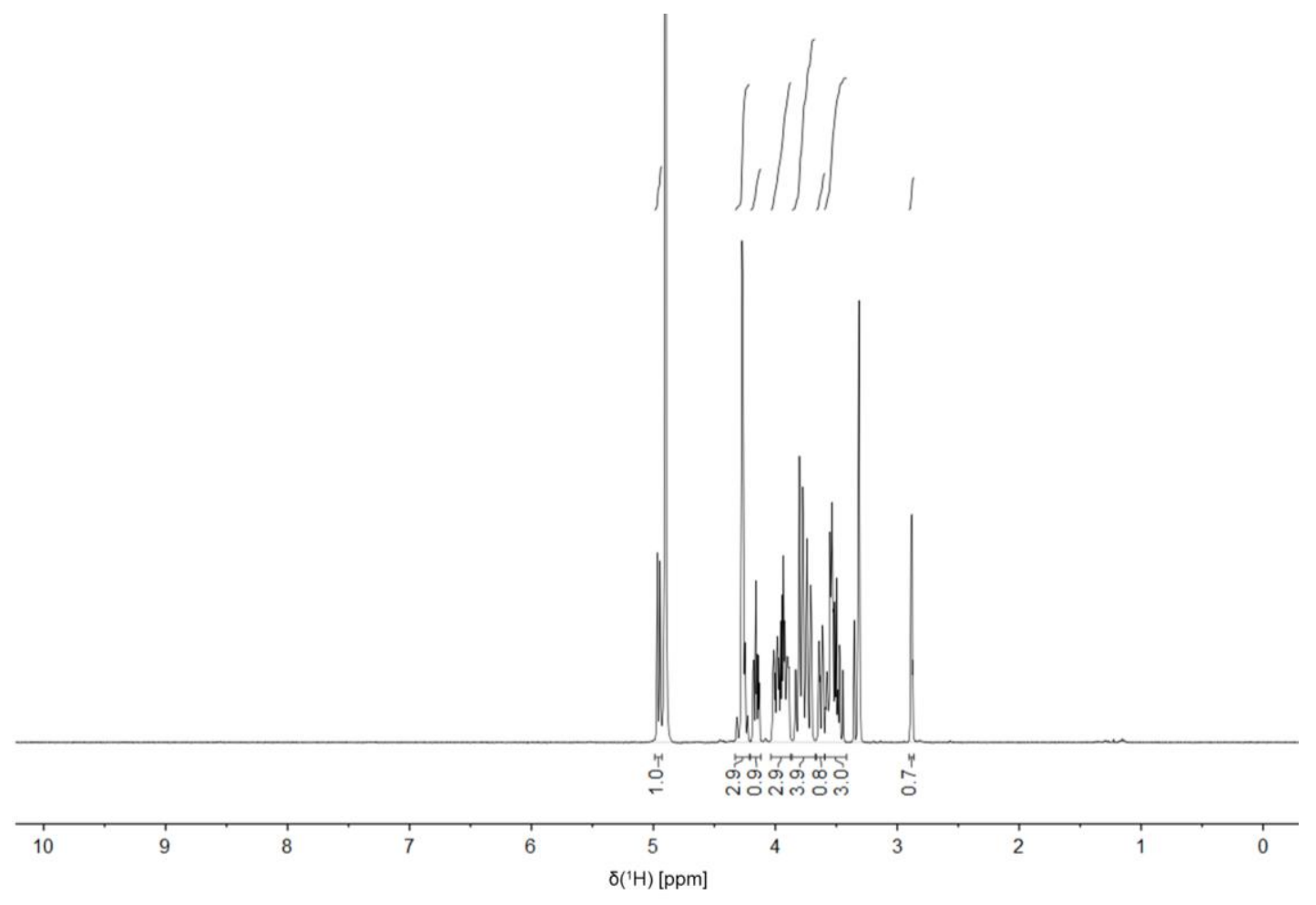

536

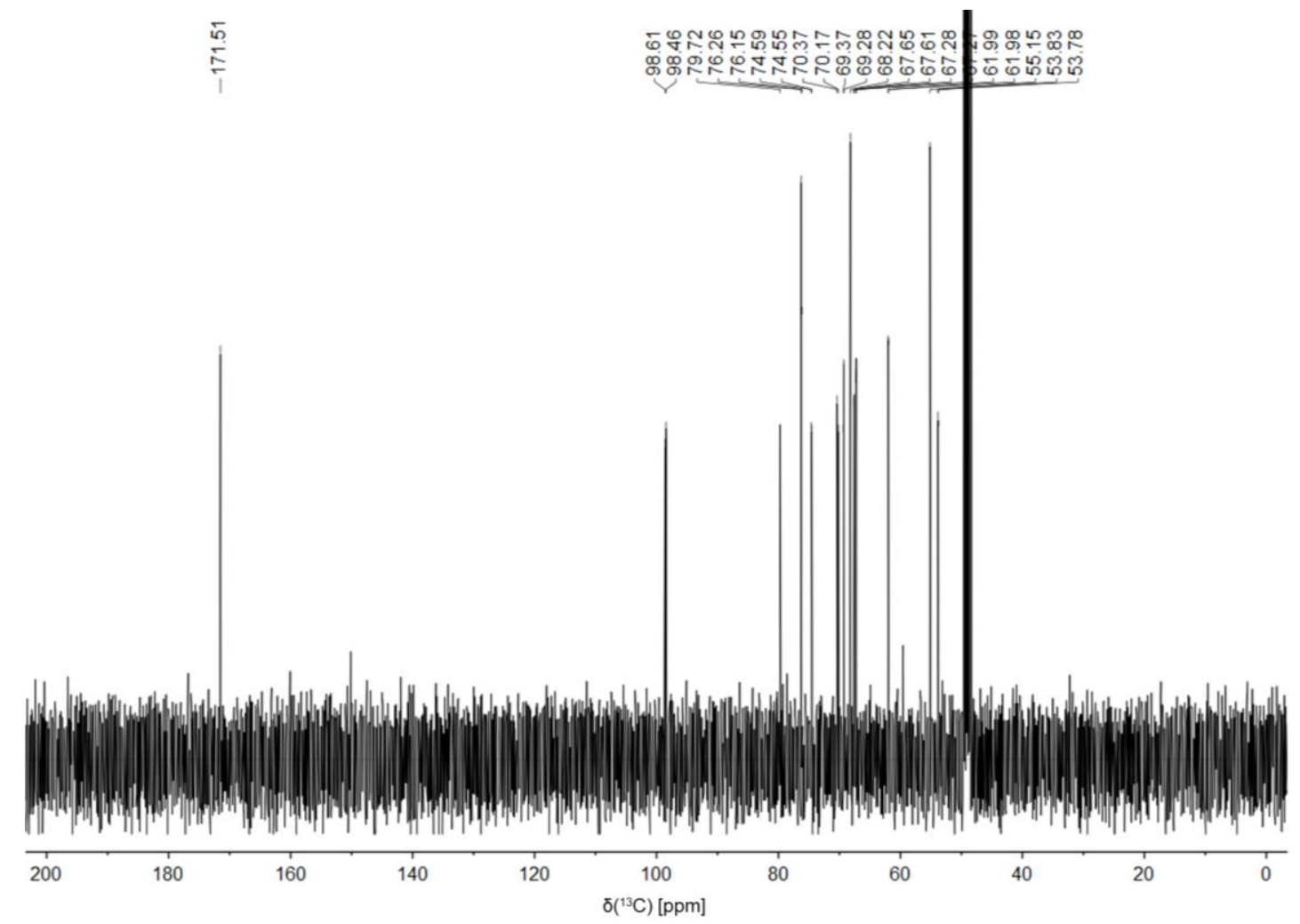

537

538 
540

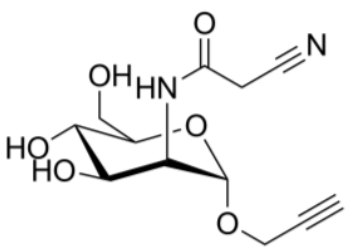

5.9

2-cyanoacetic acid (39.0 mg, $0.46 \mathrm{mmol}$ ) was dissolved in DMF (1.2 ml). Subsequently PyBOP (240 $\mathrm{mg}, 0.46 \mathrm{mmol})$ and DIPEA $(160 \mu \mathrm{l}, 0.92 \mathrm{mmol})$ were added and the mixture was stirred for $10 \mathrm{~min}$ at room temperature. $4(50 \mathrm{mg}, 0.230 \mathrm{mmol})$ was added and the mixture was stirred overnight at room temperature. Progress of the reaction was monitored by analytical TLC (10\% $\mathrm{MeOH}$ in DCM). Solvents were removed in vacuo and the residue was purified via column chromatography (gradient: hexane, DCM, $5 \% \mathrm{MeOH}$ in DCM and elution with $20 \% \mathrm{MeOH}$ in DCM). Residual impurities were removed via preparative HPLC (gradient: $\mathrm{H}_{2} \mathrm{O}$ for 10 min, from 0 to $20 \%$ acetonitrile in $\mathrm{H}_{2} \mathrm{O}$ in 30 min, from 20 to $50 \%$ acetonitrile in $\mathrm{H}_{2} \mathrm{O}$ in $10 \mathrm{~min}$, from 50 to $100 \%$ acetonitrile in $\mathrm{H}_{2} \mathrm{O}$ in 5 min and acetonitrile for $5 \mathrm{~min}$ at $\left.3.2 \mathrm{ml} \cdot \mathrm{min}^{-1}\right)$ to yield $\mathbf{5 . 9}$ as a white solid $(10.2 \mathrm{mg}, 36 \mu \mathrm{mol}, 16 \%)$.

${ }^{1} \mathrm{H}$ NMR (600.0 MHz, MeOD): $\delta=8.16$ ppm, s, $1 \mathrm{H}\left(\mathrm{NHCOCH}_{2} \mathrm{CN}\right) ; \delta=4.91 \mathrm{ppm}, \mathrm{d}, 1 \mathrm{H}, \mathrm{J}=1.1$ $\mathrm{Hz}(\mathrm{H} 1) ; \delta=4.29$ ppm, m, $1 \mathrm{H}(\mathrm{H} 2) ; \delta=4.27$ ppm, m, $2 \mathrm{H}(\mathrm{OCH} 2 \mathrm{CCH}) ; \delta=3.92 \mathrm{ppm}$, dd, $1 \mathrm{H}, \mathrm{J}=$ 4.8, $9.1 \mathrm{~Hz}(\mathrm{H} 3) ; \delta=3.81$ ppm, m, $2 \mathrm{H}(\mathrm{H} 6 \mathrm{a} / \mathrm{b}) ; \delta=3.62 \mathrm{ppm}, \mathrm{m}, 2 \mathrm{H}\left(\mathrm{NHCOCH}_{2} \mathrm{CN}\right) ; \delta=3.54 \mathrm{ppm}$, $\mathrm{m}, 2 \mathrm{H}(\mathrm{H} 4, \mathrm{H} 5) ; \delta=2.87 \mathrm{ppm}, \mathrm{t}, 1 \mathrm{H}, \mathrm{J}=2.3 \mathrm{~Hz}\left(\mathrm{OCH}_{2} \mathrm{CCH}\right)$.

${ }^{13} \mathrm{C}$ NMR (100.6 MHz, MeOD): $\delta=165.3 \mathrm{ppm}, 1 \mathrm{C}\left(\mathrm{NHCOCH}_{2} \mathrm{CN}\right) ; \delta=116.0 \mathrm{ppm}, 1 \mathrm{C}$ $\left(\mathrm{NHCOCH}_{2} \mathrm{CN}\right) ; \delta=98.7 \mathrm{ppm}, 1 \mathrm{C}(\mathrm{C} 1) ; \delta=79.7 \mathrm{ppm}, 1 \mathrm{C}\left(\mathrm{OCH}_{2} \mathrm{CCH}\right) ; \delta=76.3 \mathrm{ppm}, 1 \mathrm{C}$ $\left(\mathrm{OCH}_{2} \mathrm{CCH}\right) ; \delta=74.6$ ppm, $1 \mathrm{C}(\mathrm{C} 5) ; \delta=70.47,1 \mathrm{C}(\mathrm{C} 3) ; \delta=68.2 \mathrm{ppm}, 1 \mathrm{C}(\mathrm{C} 4) ; \delta=62.1 \mathrm{ppm}, 1 \mathrm{C}$ $(\mathrm{C} 6) ; \delta=55.1,1 \mathrm{C}\left(\mathrm{OCH}_{2} \mathrm{CCH}\right) ; \delta=54.7 \mathrm{ppm}, 1 \mathrm{C}(\mathrm{C} 2) ; \delta=26.0 \mathrm{ppm}, 1 \mathrm{C}\left(\mathrm{NHCOCH}_{2} \mathrm{CN}\right)$.

$\mathrm{R}_{\mathrm{f}}=0.25$ with $10 \%$ in $\mathrm{MeOH}$ in DCM.

HR ESI-MS for $\mathrm{C}_{12} \mathrm{H}_{16} \mathrm{~N}_{2} \mathrm{O}_{6}: \mathrm{m} \cdot \mathrm{z}^{-1}\left(\mathrm{M}+\mathrm{Na}^{+}\right)_{\text {calc }}=307.091 ; \mathrm{m} \cdot \mathrm{z}^{-1}\left(\mathrm{M}+\mathrm{Na}^{+}\right)_{\text {obs }}=307.096$. 


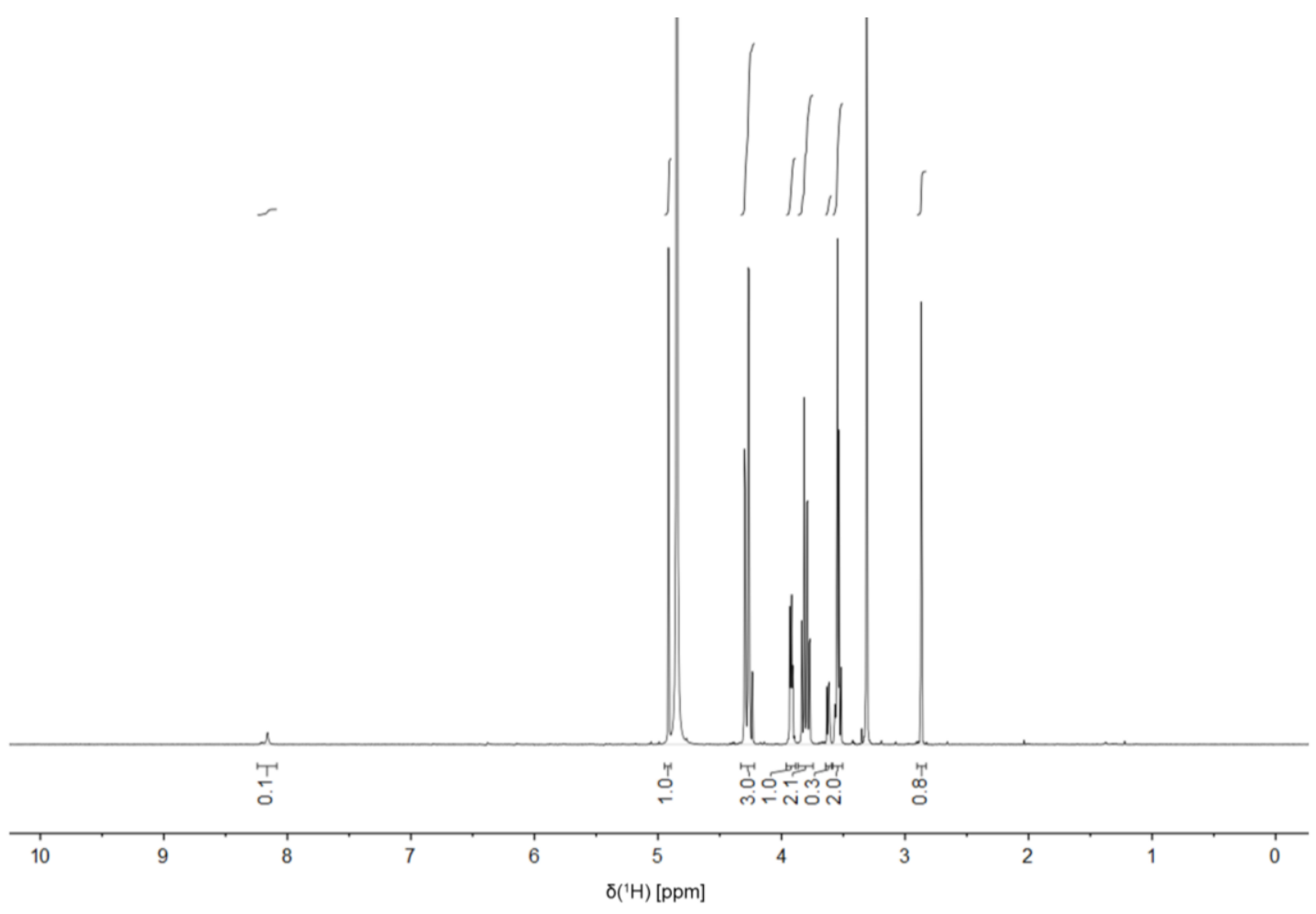

560

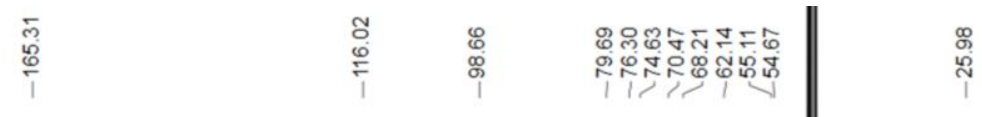

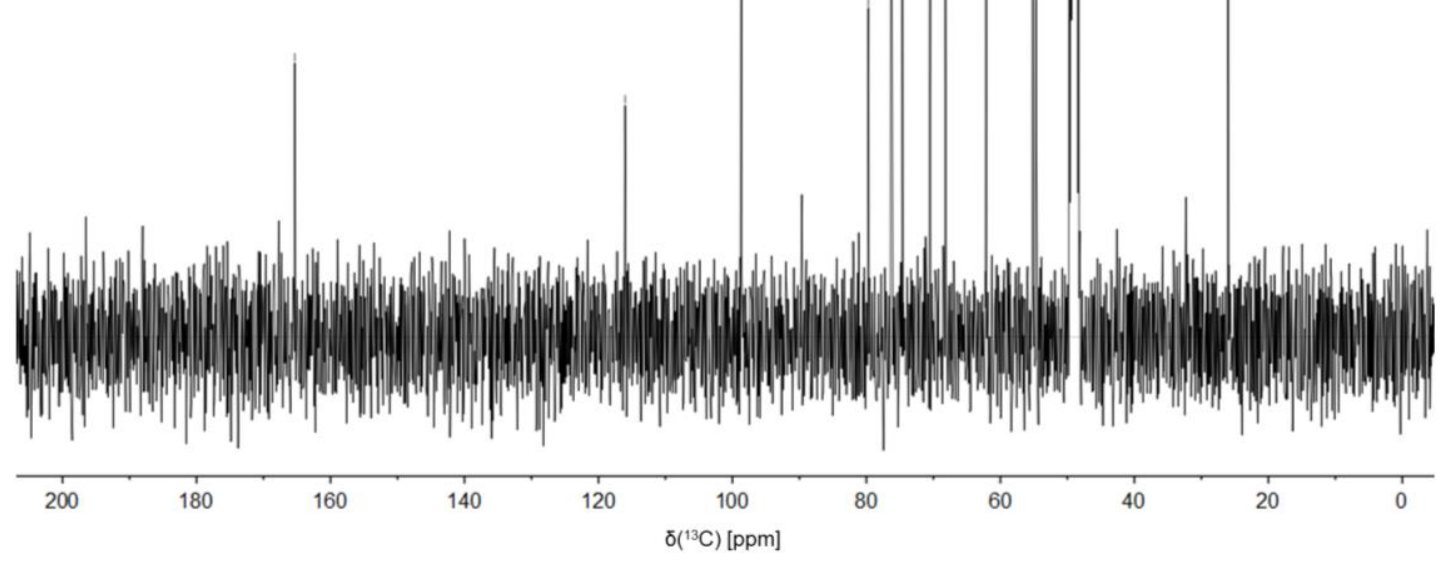

562 
564

565

566

567

568

569

570

571

572

573

574

575

576

577

578

579

580

581

582

583

584

585

586

587

588

589

590

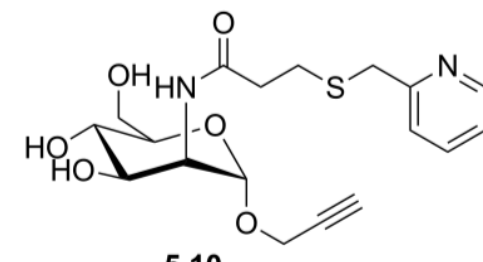

5.10

3-((Pyridin-2-ylmethyl)thio)propanoic acid (42.0 mg, $210 \mu \mathrm{mol}$, Enamine) was dissolved in DMF $(600 \mu \mathrm{l})$. Subsequently PyBOP $(110 \mathrm{mg}, 210 \mu \mathrm{mol})$ and DIPEA $(80 \mu 1,430 \mu \mathrm{mol})$ were added and the mixture was stirred for $10 \mathrm{~min}$ at room temperature. $4(23 \mathrm{mg}, 110 \mu \mathrm{mol})$ was added and the mixture was stirred overnight at room temperature. Progress of the reaction was monitored by analytical TLC $(15 \% \mathrm{MeOH}$ in $\mathrm{DCM})$. The reaction was quenched with $\mathrm{MeOH}(1 \mathrm{ml})$ and after addition of $1 \mathrm{M}$ $\mathrm{NaOH}(600 \mu \mathrm{l}, 600 \mu \mathrm{mol})$ solvents were removed in vacuo. The residue was purified via preparative HPLC (gradient: $\mathrm{H}_{2} \mathrm{O}$ for $10 \mathrm{~min}$, from 0 to $20 \%$ acetonitrile in $\mathrm{H}_{2} \mathrm{O}$ in $30 \mathrm{~min}$, from 20 to $50 \%$ acetonitrile in $\mathrm{H}_{2} \mathrm{O}$ in $10 \mathrm{~min}$, from 50 to $100 \%$ acetonitrile in $\mathrm{H}_{2} \mathrm{O}$ in $5 \mathrm{~min}$ and acetonitrile for $5 \mathrm{~min}$ at $\left.3.2 \mathrm{ml} \cdot \mathrm{min}^{-1}\right)$ to yield $\mathbf{5 . 1 0}$ as a white solid $(26.1 \mathrm{mg}, 66 \mu \mathrm{mol}, 62 \%)$.

${ }^{1} \mathrm{H}$ NMR (400.0 MHz, MeOD): $\delta=8.46 \mathrm{ppm}, \mathrm{d}, 1 \mathrm{H}, \mathrm{J}=4.8 \mathrm{~Hz}\left(\mathrm{SCH}_{2} \mathrm{CCHCHCHCHN}\right) ; \delta=8.12$ ppm, s, $1 \mathrm{H}\left(\mathrm{NHCOCH}_{2} \mathrm{CH}_{2} \mathrm{~S}\right) ; \delta=7.82 \mathrm{ppm}$, td, $1 \mathrm{H}, \mathrm{J}=1.5,7.7 \mathrm{~Hz}\left(\mathrm{SCH}_{2} \mathrm{CCHCHCHCHN}\right) ; \delta=$ $7.52 \mathrm{ppm}, \mathrm{d}, 1 \mathrm{H}, \mathrm{J}=7.9 \mathrm{~Hz}\left(\mathrm{SCH}_{2} \mathrm{CCHCHCHCHN}\right) ; \delta=7.31 \mathrm{ppm}, \mathrm{dd}, 1 \mathrm{H}, \mathrm{J}=5.2,7.4 \mathrm{~Hz}$ $\left(\mathrm{SCH}_{2} \mathrm{CCHCHCHCHN}\right) ; \delta=4.89 \mathrm{ppm}, \mathrm{s}, 1 \mathrm{H}(\mathrm{H} 1) ; \delta=4.32 \mathrm{ppm}, \mathrm{d}, 1 \mathrm{H}, \mathrm{J}=5.0 \mathrm{~Hz}(\mathrm{H} 2) ; \delta=4.26$ ppm, m, $2 \mathrm{H}(\mathrm{OCH} 2 \mathrm{CCH}) ; \delta=3.91 \mathrm{ppm}, \mathrm{dd}, 1 \mathrm{H}, \mathrm{J}=4.8,9.3 \mathrm{~Hz}(\mathrm{H} 3) ; \delta=3.87 \mathrm{ppm}, \mathrm{s}, 2 \mathrm{H}$ $\left(\mathrm{SCH}_{2} \mathrm{CCHCHCHCHN}\right) ; \delta=3.81 \mathrm{ppm}, \mathrm{m}, 2 \mathrm{H}(\mathrm{H6a} / \mathrm{b}) ; \delta=3.61 \mathrm{ppm}, \mathrm{m}, 1 \mathrm{H}(\mathrm{H} 4) ; \delta=3.54 \mathrm{ppm}, \mathrm{m}$, $1 \mathrm{H}(\mathrm{H} 5) ; \delta=2.86 \mathrm{ppm}, \mathrm{t}, 1 \mathrm{H}, \mathrm{J}=2.3 \mathrm{~Hz}\left(\mathrm{OCH}_{2} \mathrm{CCH}\right) ; \delta=2.74 \mathrm{ppm}, \mathrm{m}, 2 \mathrm{H}\left(\mathrm{NHCOCH}_{2} \mathrm{CH}_{2} \mathrm{~S}\right) ; \delta=$ $2.58 \mathrm{ppm}, \mathrm{m}, 2 \mathrm{H}\left(\mathrm{NHCOCH}_{2} \mathrm{CH}_{2} \mathrm{~S}\right)$.

${ }^{13} \mathrm{C}$ NMR (100.6 MHz, MeOD): $\delta=174.7 \mathrm{ppm}, 1 \mathrm{C}\left(\mathrm{NHCOCH}_{2} \mathrm{CH}_{2} \mathrm{~S}\right) ; \delta=159.8 \mathrm{ppm}, 1 \mathrm{C}$ $\left(\mathrm{SCH}_{2} \mathrm{CCHCHCHCHN}\right) ; \delta=149.6 \mathrm{ppm}, 1 \mathrm{C}\left(\mathrm{SCH}_{2} \mathrm{CCHCHCHCHN}\right) ; \delta=139.1 \mathrm{ppm}, 1 \mathrm{C}$ $\left(\mathrm{SCH}_{2} \mathrm{CCHCHCHCHN}\right) ; \delta=125.1 \mathrm{ppm}, 1 \mathrm{C}\left(\mathrm{SCH}_{2} \mathrm{CCHCHCHCHN}\right) ; \delta=123.7 \mathrm{ppm}, 1 \mathrm{C}$ $\left(\mathrm{SCH}_{2} \mathrm{CCHCHCHCHN}\right) ; \delta=99.0$ ppm, $1 \mathrm{C}(\mathrm{C} 1) ; \delta=79.8 \mathrm{ppm}, 1 \mathrm{C}\left(\mathrm{OCH}_{2} \mathrm{CCH}\right) ; \delta=76.2 \mathrm{ppm}, 1 \mathrm{C}$ $\left(\mathrm{OCH}_{2} \mathrm{CCH}\right) ; \delta=74.6 \mathrm{ppm}, 1 \mathrm{C}(\mathrm{C} 5) ; \delta=70.62,1 \mathrm{C}(\mathrm{C} 3) ; \delta=68.2 \mathrm{ppm}, 1 \mathrm{C}(\mathrm{C} 4) ; \delta=62.2 \mathrm{ppm}, 1 \mathrm{C}$ $(\mathrm{C} 6) ; \delta=55.1 \mathrm{ppm}, 1 \mathrm{C}\left(\mathrm{OCH}_{2} \mathrm{CCH}\right) ; \delta=54.1 \mathrm{ppm}, 1 \mathrm{C}(\mathrm{C} 2) ; \delta=37.9 \mathrm{ppm}, 1 \mathrm{C}$ $\left(\mathrm{SCH}_{2} \mathrm{CCHCHCHCHN}\right) ; \delta=36.8 \mathrm{ppm}, 1 \mathrm{C}\left(\mathrm{NHCOCH}_{2} \mathrm{CH}_{2} \mathrm{~S}\right) ; \delta=28.2 \mathrm{ppm}, 1 \mathrm{C}\left(\mathrm{NHCOCH}_{2} \mathrm{CH}_{2} \mathrm{~S}\right)$.

$\mathrm{R}_{\mathrm{f}}=0.67$ with $15 \%$ in $\mathrm{MeOH}$ in DCM.

HR ESI-MS for $\mathrm{C}_{18} \mathrm{H}_{24} \mathrm{~N}_{2} \mathrm{O}_{6} \mathrm{~S}: \mathrm{m} \cdot \mathrm{z}^{-1}\left(\mathrm{M}+\mathrm{Na}^{+}\right)_{\text {calc }}=419.125 ; \mathrm{m} \cdot \mathrm{z}^{-1}\left(\mathrm{M}+\mathrm{Na}^{+}\right)_{\text {obs }}=419.124$. 


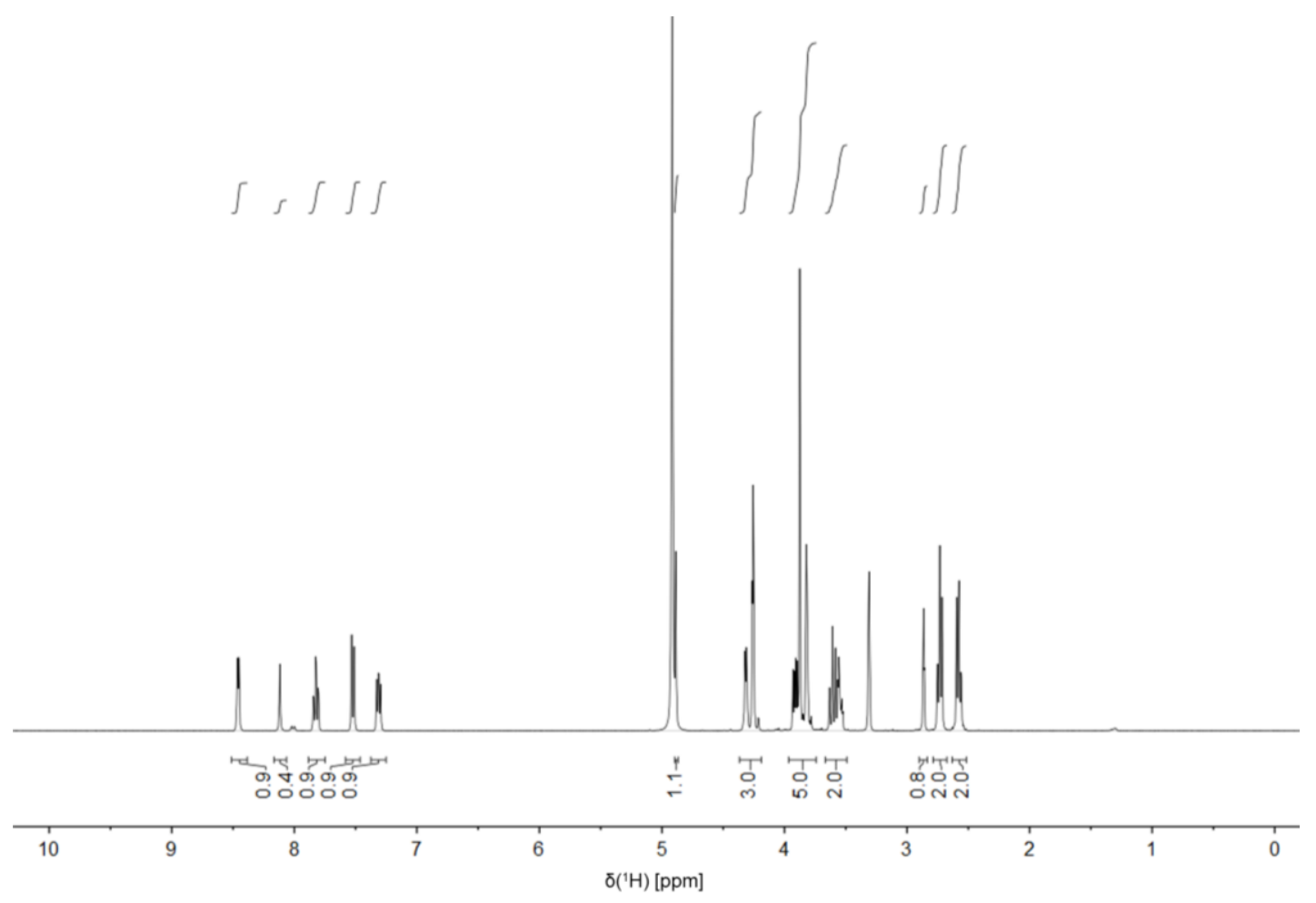

591

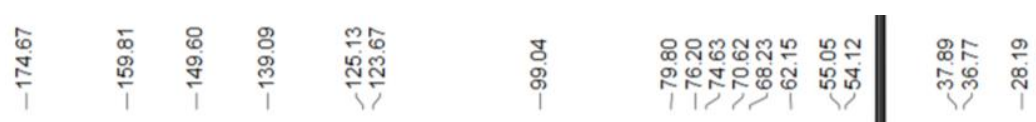

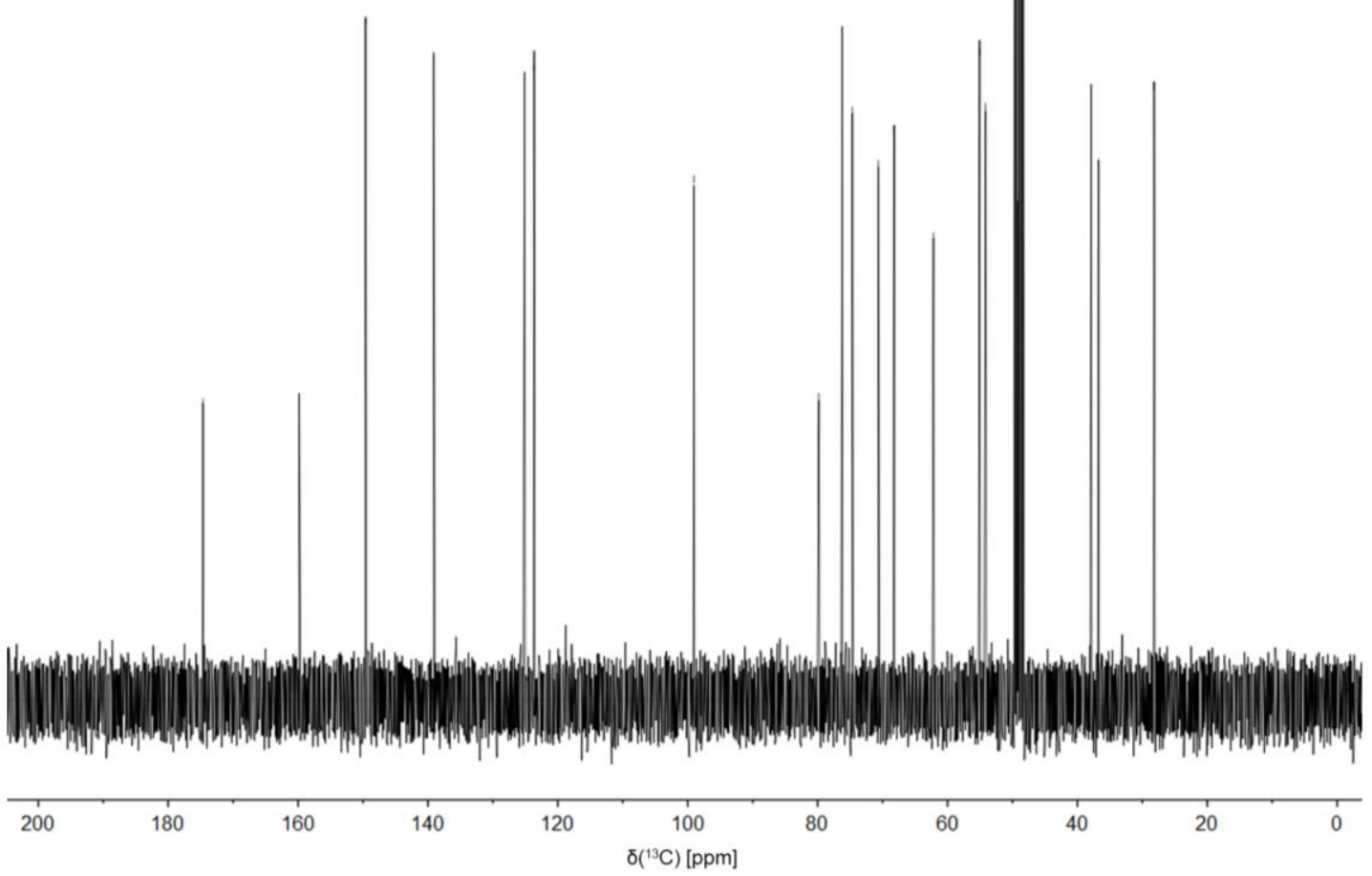


595

596

597

598

599

600

601

602

603

604

605

606

607

608

609

610

611

612

613

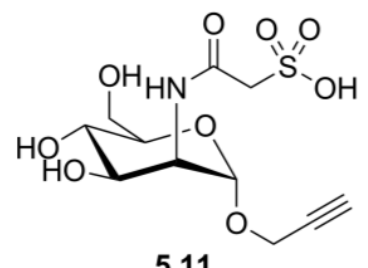

5.11

2-Sulfoacetic acid (65 mg, $460 \mu \mathrm{mol}$ ) was dissolved in DMF (1.2 ml). Subsequently, PyBOP (240 mg, $460 \mu \mathrm{mol})$ and DIPEA $(160 \mu \mathrm{l}, 920 \mu \mathrm{mol})$ were added and the mixture was stirred for $10 \mathrm{~min}$ at room temperature. $4(45 \mathrm{mg}, 230 \mu \mathrm{mol})$ was added and the mixture was stirred overnight at room temperature. Progress of the reaction was monitored by analytical TLC (20\% MeOH in DCM). After the addition of $1 \mathrm{M} \mathrm{NaOH}(1.4 \mathrm{ml}, 1.4 \mathrm{mmol})$ solvents were removed in vacuo. The residue was purified via reversed-phase column chromatography (elution with $\mathrm{H}_{2} \mathrm{O}$ ). Residual impurities were removed via preparative HPLC (gradient: $\mathrm{H}_{2} \mathrm{O}$ for $10 \mathrm{~min}$, from 0 to $100 \%$ acetonitrile in $\mathrm{H}_{2} \mathrm{O}$ in 40 $\mathrm{min}$, and acetonitrile for $10 \mathrm{~min}$ at $1.0 \mathrm{ml} \cdot \mathrm{min}^{-1}$ ) to yield $\mathbf{5 . 1 1}$ as a white solid (18 $\mathrm{mg}, 53 \mu \mathrm{mol}, 23 \%$ ).

${ }^{1} \mathrm{H}$ NMR (600.0 MHz, MeOD): $\delta=8.09$ ppm, s, $1 \mathrm{H}\left(\mathrm{NHCOCH}_{2} \mathrm{SO}_{3} \mathrm{H}\right) ; \delta=4.98 \mathrm{ppm}, \mathrm{s}, 1 \mathrm{H}(\mathrm{H} 1) ; \delta$ $=4.35 \mathrm{ppm}, \mathrm{d}, 1 \mathrm{H}, \mathrm{J}=4.2 \mathrm{~Hz}(\mathrm{H} 2) ; \delta=4.27 \mathrm{ppm}, \mathrm{m}, 2 \mathrm{H}(\mathrm{OCH} 2 \mathrm{CCH}) ; \delta=3.89 \mathrm{ppm}, \mathrm{dd}, 1 \mathrm{H}, \mathrm{J}=$ 4.5, $9.2 \mathrm{~Hz}(\mathrm{H} 3) ; \delta=3.78$ ppm, m, $3 \mathrm{H}\left(\mathrm{NHCOCH}_{2} \mathrm{SO}_{3} \mathrm{H}, \mathrm{H} 6 \mathrm{a} / \mathrm{b}\right) ; \delta=3.55$ ppm, m, $2 \mathrm{H}(\mathrm{H} 4, \mathrm{H} 5) ; \delta=$ $2.85 \mathrm{ppm}, \mathrm{t}, 1 \mathrm{H}, \mathrm{J}=2.4 \mathrm{~Hz}\left(\mathrm{OCH}_{2} \mathrm{CCH}\right)$.

${ }^{13} \mathrm{C}$ NMR (100.6 MHz, MeOD): $\delta=168.2$ ppm, $1 \mathrm{C}\left(\mathrm{NHCOCH}_{2} \mathrm{SO}_{3} \mathrm{H}\right) ; \delta=98.7 \mathrm{ppm}, 1 \mathrm{C}(\mathrm{C} 1) ; \delta=$ $79.8 \mathrm{ppm}, 1 \mathrm{C}\left(\mathrm{OCH}_{2} \mathrm{CCH}\right) ; \delta=76.2 \mathrm{ppm}, 1 \mathrm{C}\left(\mathrm{OCH}_{2} \mathrm{CCH}\right) ; \delta=74.8 \mathrm{ppm}, 1 \mathrm{C}(\mathrm{C} 5) ; \delta=71.1 \mathrm{ppm}, 1$ $\mathrm{C}(\mathrm{C} 3) ; \delta=68.6 \mathrm{ppm}, 1 \mathrm{C}(\mathrm{C} 4) ; \delta=62.5 \mathrm{ppm}, 1 \mathrm{C}(\mathrm{C} 6) ; \delta=58.1 \mathrm{ppm}, 1 \mathrm{C}\left(\mathrm{NHCOCH}_{2} \mathrm{SO}_{3} \mathrm{H}\right) ; \delta=$ $55.1 \mathrm{ppm}, 1 \mathrm{C}\left(\mathrm{OCH}_{2} \mathrm{CCH}\right) ; \delta=54.4 \mathrm{ppm}, 1 \mathrm{C}(\mathrm{C} 2)$.

$\mathrm{R}_{\mathrm{f}}=0.04$ with $20 \%$ in $\mathrm{MeOH}$ in DCM.

HR ESI-MS for $\mathrm{C}_{11} \mathrm{H}_{17} \mathrm{NO}_{9} \mathrm{~S}: \mathrm{m} \cdot \mathrm{z}^{-1}\left(\mathrm{M}-\mathrm{H}^{+}\right)_{\text {calc }}=338.054 ; \mathrm{m} \cdot \mathrm{z}^{-1}\left(\mathrm{M}-\mathrm{H}^{+}\right)_{\mathrm{obs}}=338.058$. 


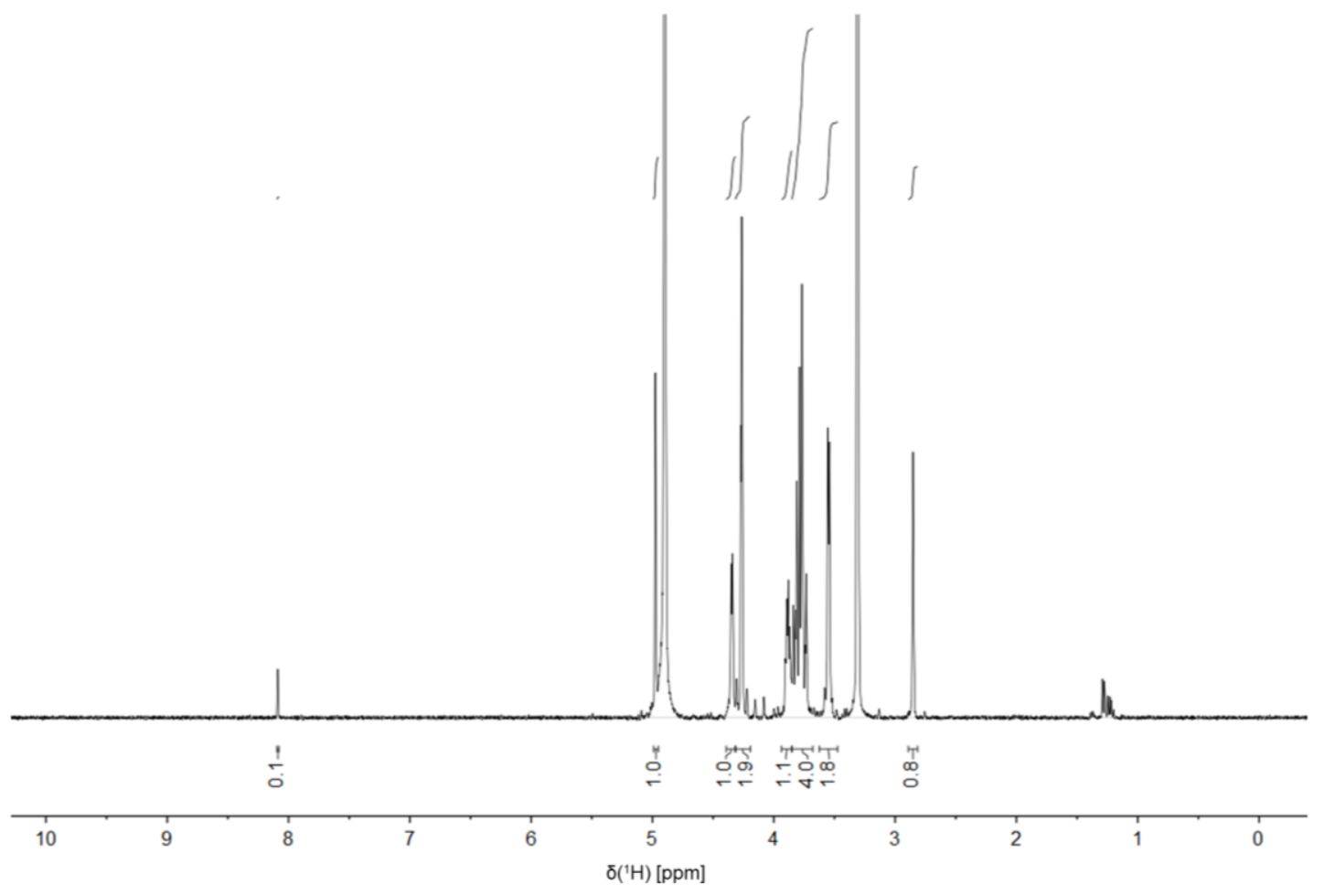

614
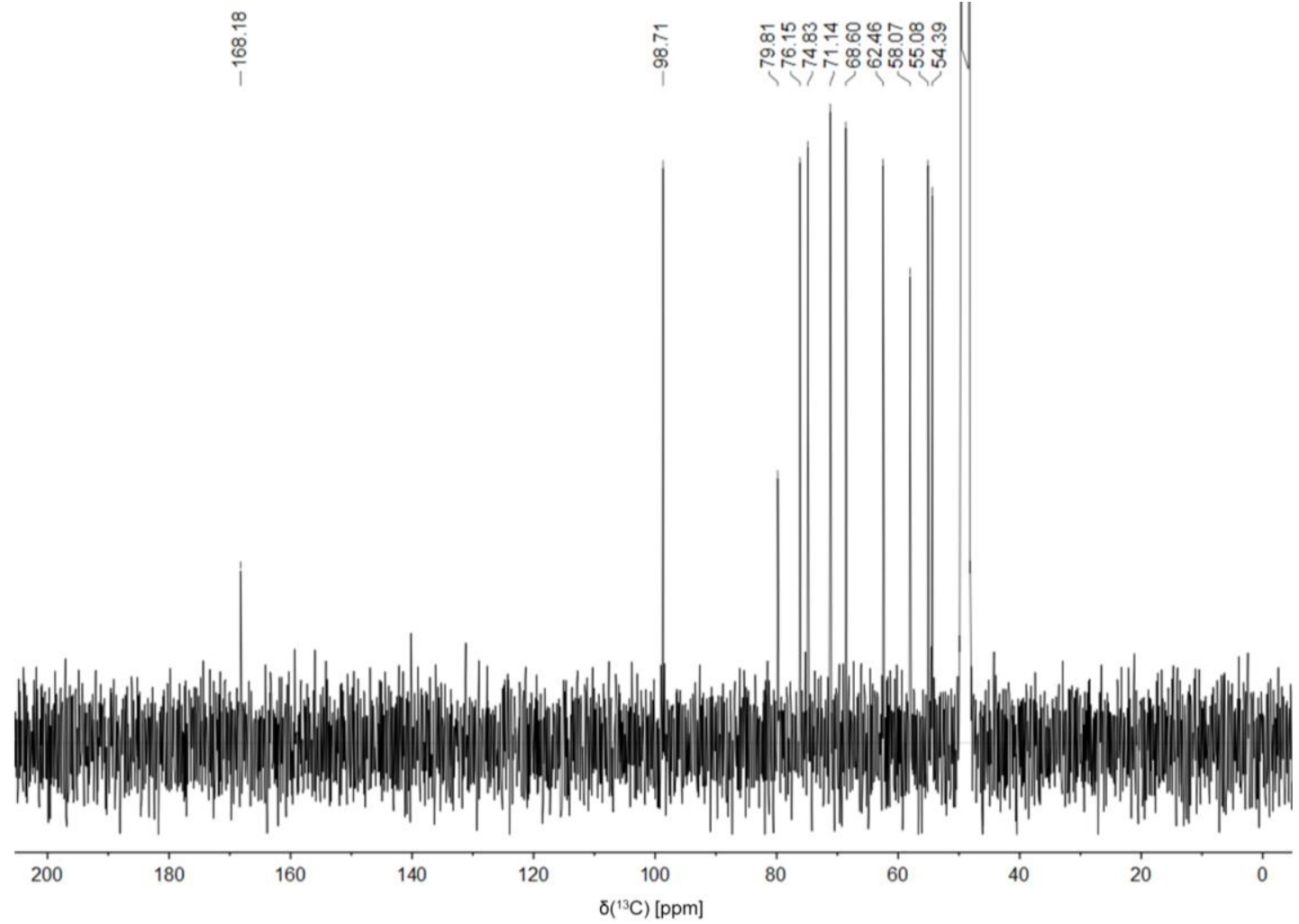

615

616 
618

619

620

621

622

623

624

625

626

627

628

629

630

631

632

633

634

635

636

637

638

639

640

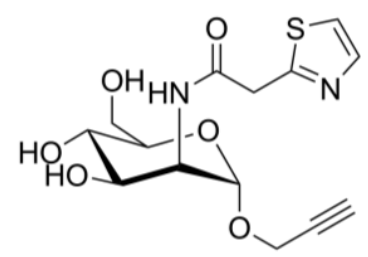

5.12

2-(Thiazol-2-yl)acetic acid $(50.0 \mathrm{mg}, 350 \mu \mathrm{mol})$ was dissolved in DMF (0.9 ml). Subsequently, PyBOP $(180 \mathrm{mg}, 350 \mu \mathrm{mol})$ and DIPEA $(120 \mu \mathrm{l}, 700 \mu \mathrm{mol})$ were added and the mixture was stirred for $10 \mathrm{~min}$ at room temperature. $16 \mathrm{~h}$ after the addition of 4 (38 $\mathrm{mg}, 180 \mu \mathrm{mol})$, more PyBOP $(180 \mathrm{mg}$, $350 \mu \mathrm{mol})$, DIPEA $(120 \mu \mathrm{l}, 700 \mu \mathrm{mol})$ and 2-(thiazol-2-yl)acetic acid $(50 \mathrm{mg}, 350 \mu \mathrm{mol})$ were added. The mixture was stirred overnight at room temperature and progress of the reaction was monitored by analytical TLC (10\% MeOH in DCM). After the addition of $1 \mathrm{M} \mathrm{NaOH}$ (1.4 ml, $1.4 \mathrm{mmol})$ solvents were removed in vacuo. The residue was purified via reversed-phase column chromatography (elution with $\mathrm{H}_{2} \mathrm{O}$ ). Residual impurities were removed via preparative HPLC (gradient: $\mathrm{H}_{2} \mathrm{O}$ for $10 \mathrm{~min}$, from 0 to $20 \%$ acetonitrile in $\mathrm{H}_{2} \mathrm{O}$ in $30 \mathrm{~min}$, from 20 to $50 \%$ acetonitrile in $\mathrm{H}_{2} \mathrm{O}$ in $10 \mathrm{~min}$, from 50 to $100 \%$ acetonitrile in $\mathrm{H}_{2} \mathrm{O}$ in $5 \mathrm{~min}$ and acetonitrile for $5 \mathrm{~min}$ at $3.2 \mathrm{ml} \cdot \mathrm{min}^{-1}$ ) to yield $\mathbf{5 . 1 2}$ as a white solid (5.2 mg, $15 \mu \mathrm{mol}, 9 \%)$.

${ }^{1} \mathrm{H}$ NMR (600.0 MHz, MeOD): $\delta=4.97$ ppm, d, $1 \mathrm{H}, \mathrm{J}=1.2 \mathrm{~Hz}(\mathrm{H} 1) ; \delta=4.30 \mathrm{ppm}$, dd, $1 \mathrm{H}, \mathrm{J}=1.3$, $4.8 \mathrm{~Hz}(\mathrm{H} 2) ; \delta=4.28$ ppm, m, $2 \mathrm{H}(\mathrm{OCH} 2 \mathrm{CCH}) ; \delta=4.02 \mathrm{ppm}, \mathrm{s}, 2 \mathrm{H}\left(\mathrm{NHCOCH}_{2} \mathrm{CNCCS}\right) ; \delta=3.96$ ppm, dd, $1 \mathrm{H}, \mathrm{J}=4.8,8.8 \mathrm{~Hz}(\mathrm{H} 3) ; \delta=3.83$ ppm, m, $1 \mathrm{H}(\mathrm{H} 6 \mathrm{a}) ; \delta=3.75$ ppm, m, $1 \mathrm{H}(\mathrm{H} 6 \mathrm{~b}) ; \delta=3.57$ ppm, m, $1 \mathrm{H}(\mathrm{H} 5) ; \delta=3.53$ ppm, m, $1 \mathrm{H}(\mathrm{H} 4) ; \delta=2.87$ ppm, t, $1 \mathrm{H}, \mathrm{J}=2.4 \mathrm{~Hz}\left(\mathrm{OCH}_{2} \mathrm{CCH}\right)$.

${ }^{13} \mathrm{C} \mathrm{NMR}(100.6 \mathrm{MHz}, \mathrm{MeOD}): \delta=175.5 \mathrm{ppm}, 1 \mathrm{C}\left(\mathrm{NHCOCH}_{2} \mathrm{CNCCS}\right) ; \delta=98.6 \mathrm{ppm}, 1 \mathrm{C}(\mathrm{C} 1) ; \delta=$ 79.7 ppm, $1 \mathrm{C}\left(\mathrm{OCH}_{2} \mathrm{CCH}\right) ; \delta=76.2 \mathrm{ppm}, 1 \mathrm{C}\left(\mathrm{OCH}_{2} \mathrm{CCH}\right) ; \delta=74.6 \mathrm{ppm}, 1 \mathrm{C}(\mathrm{C} 5) ; \delta=70.4 \mathrm{ppm}, 1$ $\mathrm{C}(\mathrm{C} 3) ; \delta=68.4 \mathrm{ppm}, 1 \mathrm{C}(\mathrm{C} 4) ; \delta=62.6 \mathrm{ppm}, 1 \mathrm{C}\left(\mathrm{NHCOCH}_{2} \mathrm{CNCCS}\right) ; \delta=62.1 \mathrm{ppm}, 1 \mathrm{C}(\mathrm{C} 6) ; \delta=$ $55.1 \mathrm{ppm}, 1 \mathrm{C}\left(\mathrm{OCH}_{2} \mathrm{CCH}\right) ; \delta=53.8 \mathrm{ppm}, 1 \mathrm{C}(\mathrm{C} 2)$.

The aromatic carbon atoms of the thiazolyl were not detected in the conducted ${ }^{13} \mathrm{C}$ NMR experiments.

$\mathrm{R}_{\mathrm{f}}=0.24$ with $10 \%$ in $\mathrm{MeOH}$ in DCM.

HR ESI-MS for $\mathrm{C}_{14} \mathrm{H}_{18} \mathrm{~N}_{2} \mathrm{O}_{6} \mathrm{~S}: \mathrm{m} \cdot \mathrm{z}^{-1}\left(\mathrm{M}+\mathrm{Na}^{+}\right)_{\text {calc }}=365.078 ; \mathrm{m} \cdot \mathrm{z}^{-1}\left(\mathrm{M}+\mathrm{Na}^{+}\right)_{\mathrm{obs}}=365.076$. 


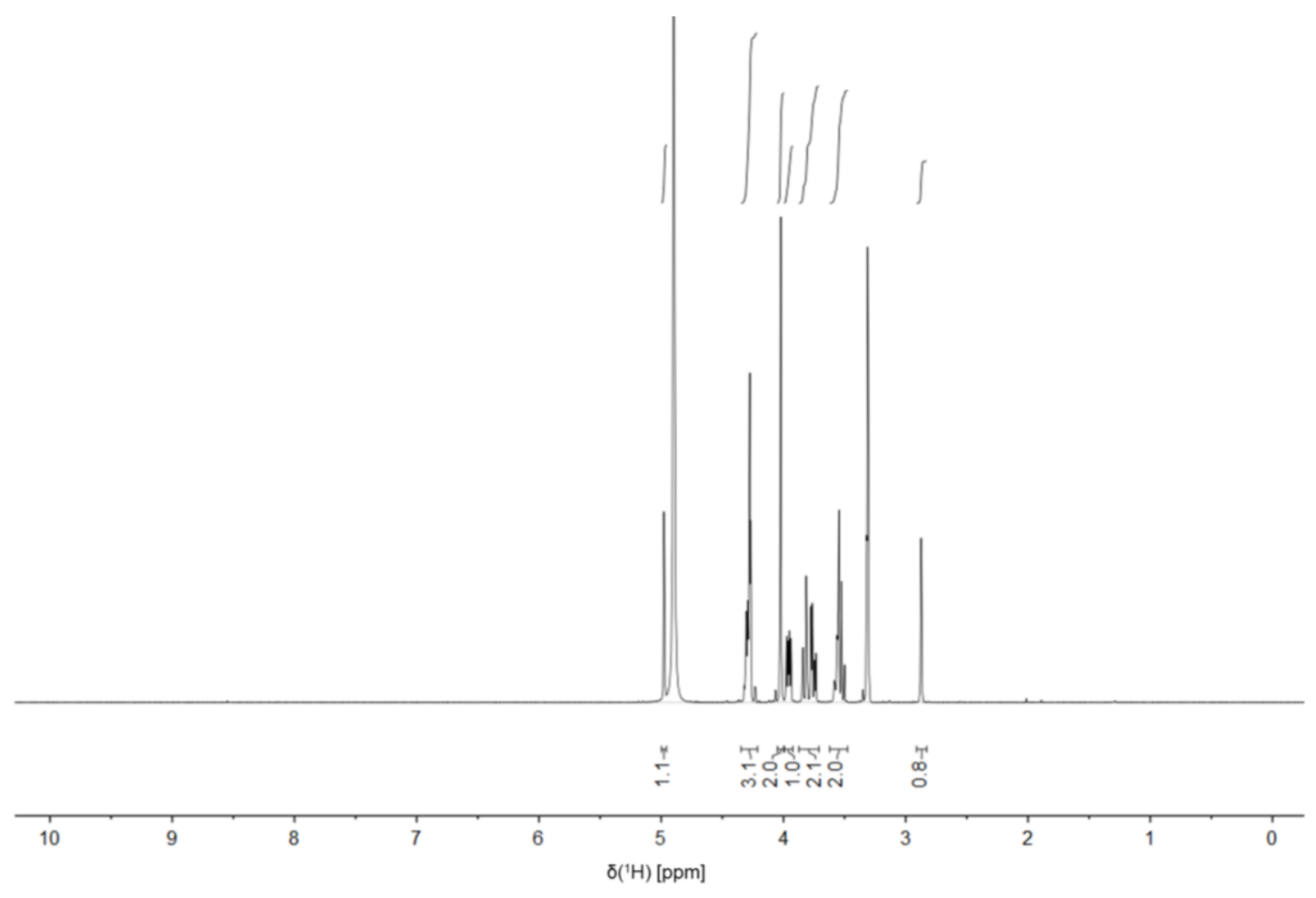

641

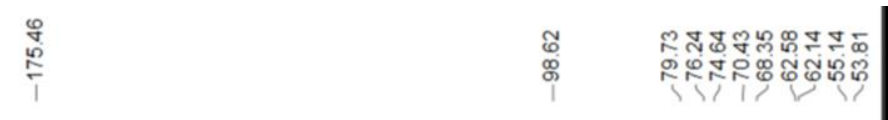

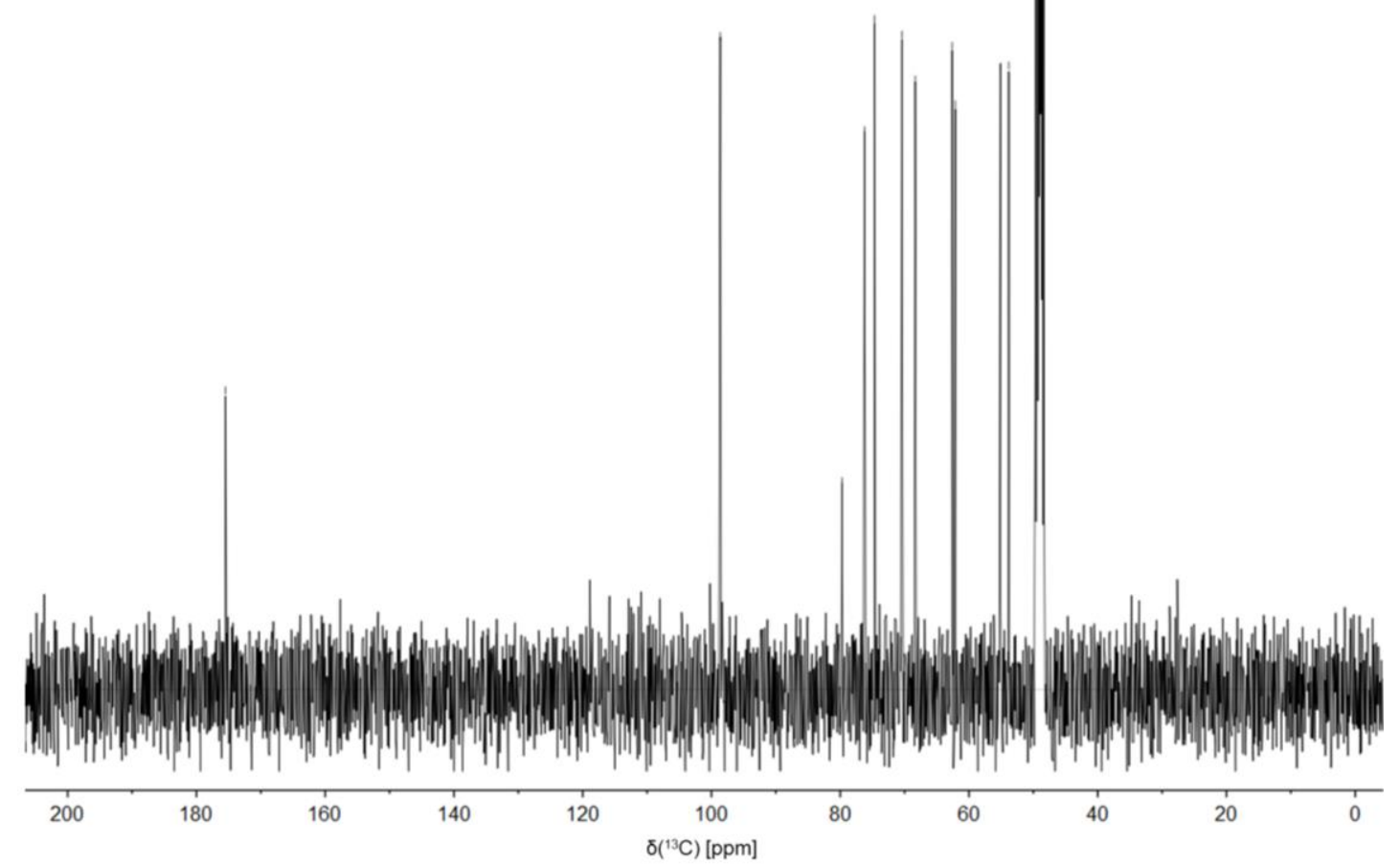

642

643 
645

646

647

648

649

650

651

652

653

654

655

656

657

658

659

660

661

662

663

664

665

666

667

668

669

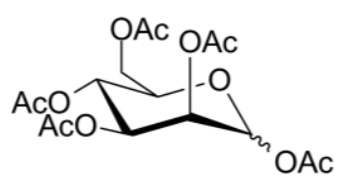

6

6 was prepared as previously published (21). Mannose (5.0 g, $28 \mathrm{mmol})$ and acetic anhydride (42 $\mathrm{ml}$, $440 \mathrm{mmol})$ were dissolved in pyridine $(100 \mathrm{ml})$. The reaction mixture was stirred over night at $50^{\circ} \mathrm{C}$. Progress of the reaction was monitored by TLC in toluene:ethyl acetate (2:1). Solvents were evaporated in vacuo and the residue was taken up in chloroform $(250 \mathrm{ml})$. The organic phase was extracted with $1 \mathrm{M} \mathrm{HCl}$, saturated $\mathrm{NaHCO}_{3}$ and $\mathrm{H}_{2} \mathrm{O}$. Subsequently, the organic phase was dried with $\mathrm{MgSO}_{4}$. Solvents were evaporated in in vacuo and the residue was purified via column chromatography (toluene:ethyl acetate (6:1)) to afford an $\alpha / \beta$-anomer mixture of 6 (7.20 g, 18.45 mmol, $80 \%)$ as a white solid.

${ }^{1} \mathrm{H}$ NMR (400.0 MHz, $\mathrm{CDCl}_{3}, \alpha$-anomer): $\delta=6.05$ ppm, m, $1 \mathrm{H}(\mathrm{H} 1) ; \delta=5.30 \mathrm{ppm}, \mathrm{m}, 2 \mathrm{H}(\mathrm{H} 3$, $\mathrm{H} 4) ; \delta=5.22, \mathrm{~m}, 1 \mathrm{H}(\mathrm{H} 2) ; \delta=4.23 \mathrm{ppm}, \mathrm{dd}, 1 \mathrm{H}, \mathrm{J}=4.7,12.1 \mathrm{~Hz}(\mathrm{H} 6 \mathrm{a}) ; \delta=4.05, \mathrm{~m}, 1 \mathrm{H}$ (H6b); $\delta=$ 4.01 ppm, m, $1 \mathrm{H}(\mathrm{H} 5) ; \delta=2.17$ - 1.96 ppm, m, $15 \mathrm{H}\left(5\right.$ times $\left.\mathrm{OCOCH}_{3}\right)$.

${ }^{13} \mathrm{C}$ NMR (100.6 MHz, $\mathrm{CDCl}_{3}, \alpha$-anomer): $\delta=170.6 \mathrm{ppm}, 1 \mathrm{C}\left(\mathrm{OCOCH}_{3}\right) ; \delta=170.0,1 \mathrm{C}\left(\mathrm{OCOCH}_{3}\right)$; $\delta=169.7 \mathrm{ppm}\left(\mathrm{OCOCH}_{3}\right) ; \delta=169.5 \mathrm{ppm}, 1 \mathrm{C}\left(\mathrm{OCOCH}_{3}\right) ; \delta=168.1 \mathrm{ppm}, 1 \mathrm{C}\left(\mathrm{OCOCH}_{3}\right) ; \delta=90.6$ ppm, $1 \mathrm{C}(\mathrm{C} 1) ; \delta=70.6 \mathrm{ppm}, 1 \mathrm{C}(\mathrm{C} 5) ; \delta=68.8 \mathrm{ppm}, 1 \mathrm{C}(\mathrm{C} 3) ; \delta=68.4 \mathrm{ppm}, 1 \mathrm{C}(\mathrm{C} 2) ; \delta=65.6$ ppm, $1 \mathrm{C}(\mathrm{C} 4) ; \delta=62.1,1 \mathrm{C}(\mathrm{C} 6) ; \delta=20.9$ ppm, $1 \mathrm{C}\left(\mathrm{OCOCH}_{3}\right) ; \delta=20.8 \mathrm{ppm}, 1 \mathrm{C}\left(\mathrm{OCOCH}_{3}\right) ; \delta=$ 20.7 ppm, $3 \mathrm{C}$ (three times $\mathrm{OCOCH}_{3}$ ).

${ }^{1} \mathrm{H}$ NMR (400.0 MHz, $\mathrm{CDCl}_{3}, \beta$-anomer): $\delta=5.83$ ppm, m, $1 \mathrm{H}(\mathrm{H} 1) ; \delta=5.44$ ppm, m, $1 \mathrm{H}(\mathrm{H} 2) ; \delta=$ $5.25 \mathrm{ppm}, \mathrm{m}, 1 \mathrm{H}(\mathrm{H} 4) ; \delta=5.10$, dd, $1 \mathrm{H}, \mathrm{J}=2.5,10.2 \mathrm{~Hz}(\mathrm{H} 3) ; \delta=4.27 \mathrm{ppm}, \mathrm{m}, 1 \mathrm{H}(\mathrm{H} 6 \mathrm{a}) ; \delta=4.05$, $\mathrm{m}, 1 \mathrm{H}(\mathrm{H} 6 \mathrm{~b}) ; \delta=3.77, \mathrm{~m}, 1 \mathrm{H}(\mathrm{H} 5) ; \delta=2.17-1.96 \mathrm{ppm}, \mathrm{m}, 15 \mathrm{H}$ (five times $\mathrm{OCOCH}_{3}$ ).

${ }^{13} \mathrm{C} \mathrm{NMR}\left(100.6 \mathrm{MHz}, \mathrm{CDCl}_{3}, \beta\right.$-anomer): $\delta=170.6 \mathrm{ppm}, 1 \mathrm{C}\left(\mathrm{OCOCH}_{3}\right) ; \delta=170.2,1 \mathrm{C}\left(\mathrm{OCOCH}_{3}\right)$; $\delta=169.8 \mathrm{ppm}\left(\mathrm{OCOCH}_{3}\right) ; \delta=169.6 \mathrm{ppm}, 1 \mathrm{C}\left(\mathrm{OCOCH}_{3}\right) ; \delta=168.4 \mathrm{ppm}, 1 \mathrm{C}\left(\mathrm{OCOCH}_{3}\right) ; \delta=90.5$ ppm, $1 \mathrm{C}(\mathrm{C} 1) ; \delta=73.3 \mathrm{ppm}, 1 \mathrm{C}(\mathrm{C} 5) ; \delta=70.7 \mathrm{ppm}, 1 \mathrm{C}(\mathrm{C} 3) ; \delta=68.2 \mathrm{ppm}, 1 \mathrm{C}(\mathrm{C} 2) ; \delta=65.5$ ppm, $1 \mathrm{C}(\mathrm{C} 4) ; \delta=62.1,1 \mathrm{C}(\mathrm{C} 6) ; \delta=20.8-20.6 \mathrm{ppm}, 5 \mathrm{C}$ (five times $\mathrm{OCOCH}_{3}$ ).

$\mathrm{R}_{\mathrm{f}}=0.43$ with toluene:ethyl acetate (3:1). 


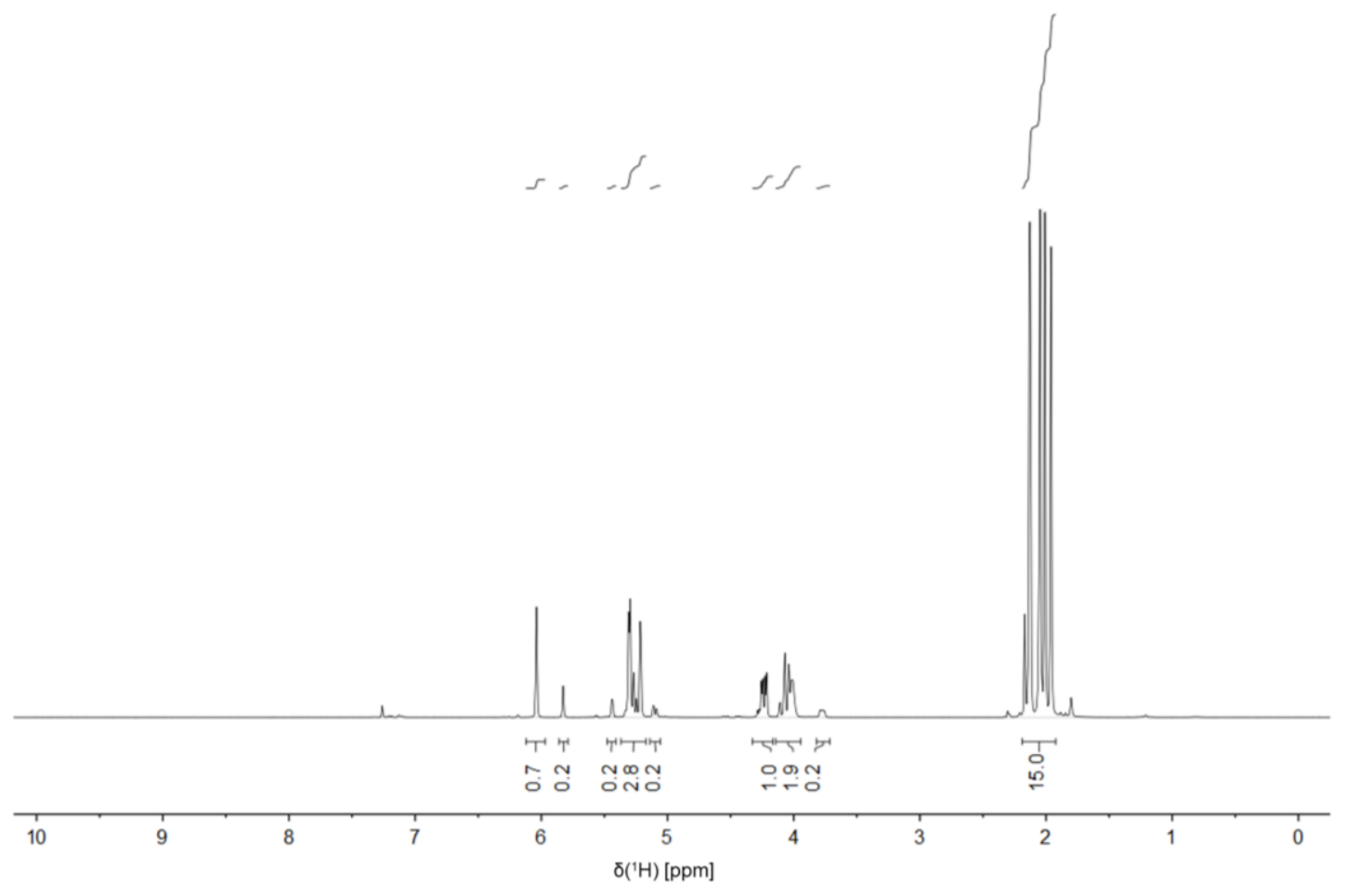

670

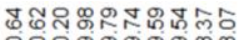

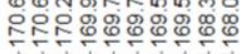

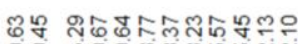

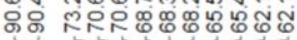

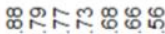

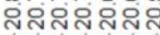

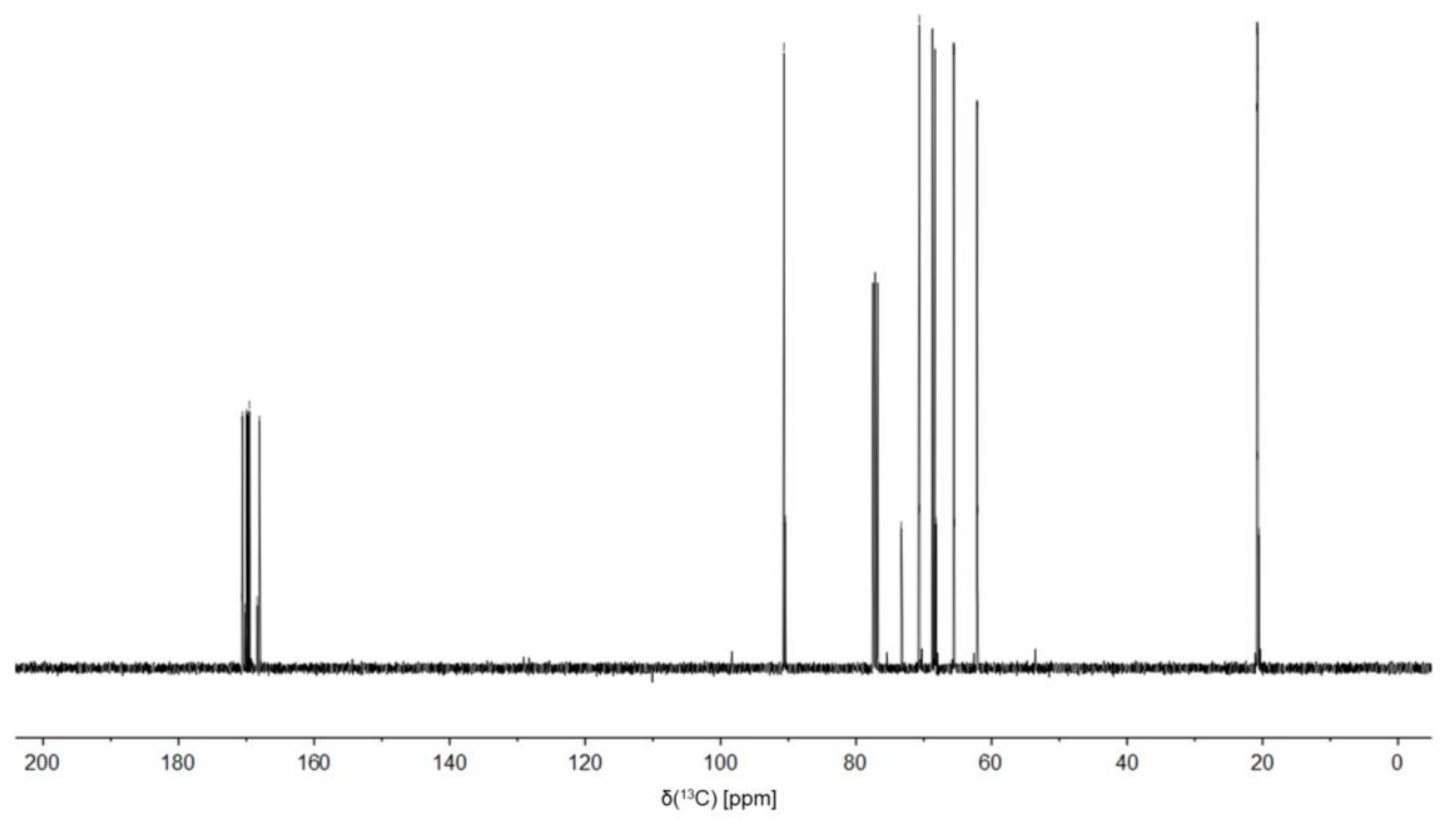

671

672 
674

675

676

677

678

679

680

681

682

683

684

685

686

687

688

689

690

691

692

693

694

695

696

697

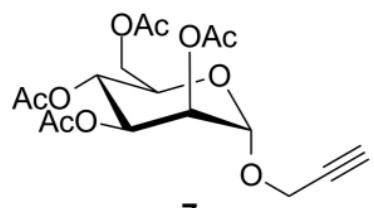

7

$6(7.20 \mathrm{~g}, 19 \mathrm{mmol})$ was dissolved in anhydrous DCM:ether $(189 \mathrm{ml}, 2: 1)$. The mixture was stirred at $0^{\circ} \mathrm{C}$ and kept under argon. Propargyl alcohol $(4.4 \mathrm{ml}, 73.8 \mathrm{mmol})$ and $\mathrm{BF}_{3} \cdot \mathrm{OEt}_{2}(4.7 \mathrm{ml}, 36.9 \mathrm{mmol})$ were added and the reaction was allowed to heat up to room temperature and stirred. After $24 \mathrm{~h}$ additional propargyl alcohol $(4.4 \mathrm{ml}, 73.8 \mathrm{mmol})$ and $\mathrm{BF}_{3} \cdot \mathrm{OEt}_{2}(4.7 \mathrm{ml}, 36.9 \mathrm{mmol})$ were added at $0^{\circ} \mathrm{C}$ and the reaction was stirred for another $40 \mathrm{~h}$. Progress of the reaction was monitored by analytical TLC (toluene:ethyl acetate (3:1)). The mixture was diluted in DCM (500 ml) and the organic phase was extracted with saturated $\mathrm{NaHCO}_{3}$ and $\mathrm{H}_{2} \mathrm{O}$. Subsequently, the organic phase was dried with $\mathrm{MgSO}_{4}$. Solvents were evaporated in vacuo and the residue was purified via column chromatography (toluene:ethyl acetate $(8: 1))$ to yield 7 (188 $\mathrm{mg}, 487 \mu \mathrm{mol}, 3 \%)$ as a light yellow resin. Starting material 2 that was not converted was recovered.

${ }^{1} \mathrm{H}$ NMR (400.0 MHz, CDCl $): \delta=5.17 \mathrm{ppm}, \mathrm{d}, 1 \mathrm{H}, \mathrm{J}=1.1 \mathrm{~Hz}(\mathrm{H} 3) ; \delta=5.15 \mathrm{ppm}, \mathrm{m}(\mathrm{H} 4) ; \delta=5.11$ ppm, m, $1 \mathrm{H}(\mathrm{H} 2) ; \delta=4.87$ ppm, d, $1 \mathrm{H}, \mathrm{J}=1.3 \mathrm{~Hz}(\mathrm{H} 1) ; \delta=4.16$ ppm, m, $1 \mathrm{H}(\mathrm{H} 6 \mathrm{a}) ; \delta=4.11 \mathrm{ppm}$, $\mathrm{d}, 2 \mathrm{H}, \mathrm{J}=2.4 \mathrm{~Hz}\left(\mathrm{OCH}_{2} \mathrm{CCH}\right) ; \delta=4.27 \mathrm{ppm}, \mathrm{dd}, 1 \mathrm{H}, \mathrm{J}=2.3,12.2 \mathrm{~Hz}(\mathrm{H} 6 \mathrm{~b}) ; \delta=3.86 \mathrm{ppm}$, ddd, 1 $\mathrm{H}, \mathrm{J}=2.3,5.3,9.3 \mathrm{~Hz}(\mathrm{H} 5) ; \delta=2.32, \mathrm{t}, 1 \mathrm{H}, \mathrm{J}=2.45 \mathrm{z}\left(\mathrm{OCH}_{2} \mathrm{CCH}\right) ; \delta=2.00 \mathrm{ppm}, \mathrm{s}, 3 \mathrm{H}\left(\mathrm{OCOCH}_{3}\right)$; $\delta=1.94 \mathrm{ppm}, \mathrm{s}, 3 \mathrm{H}\left(\mathrm{OCOCH}_{3}\right) ; \delta=1.88 \mathrm{ppm}, \mathrm{s}, 3 \mathrm{H}\left(\mathrm{OCOCH}_{3}\right) ; \delta=1.83 \mathrm{ppm}, \mathrm{s}, 3 \mathrm{H}\left(\mathrm{OCOCH}_{3}\right)$.

${ }^{13} \mathrm{C} \mathrm{NMR}(100.6 \mathrm{MHz}, \mathrm{CDCl} 3): \delta=170.7 \mathrm{ppm}, 1 \mathrm{C}\left(\mathrm{OCOCH}_{3}\right) ; \delta=170.0 \mathrm{ppm}, 1 \mathrm{C}\left(\mathrm{OCOCH}_{3}\right) ; \delta=$ $169.9 \mathrm{ppm}, 1 \mathrm{C}\left(\mathrm{OCOCH}_{3}\right) ; \delta=169.8 \mathrm{ppm}, 1 \mathrm{C}\left(\mathrm{OCOCH}_{3}\right) ; \delta=95.5 \mathrm{ppm}, 1 \mathrm{C}(\mathrm{C} 1) ; \delta=96.3 \mathrm{ppm}, 1$ $\mathrm{C}(\mathrm{C} 1) ; \delta=78.0 \mathrm{ppm}, 1 \mathrm{C}\left(\mathrm{OCH}_{2} \mathrm{CCH}\right) ; \delta=75.7 \mathrm{ppm}, 1 \mathrm{C}\left(\mathrm{OCH}_{2} \mathrm{CCH}\right) ; \delta=69.5 \mathrm{ppm}, 1 \mathrm{C}(\mathrm{C} 2) ; \delta=$ $69.1 \mathrm{ppm}, 1 \mathrm{C}(\mathrm{C} 5) ; \delta=69.0 \mathrm{ppm}, 1 \mathrm{C}(\mathrm{C} 3) ; \delta=66.1 \mathrm{ppm}, 1 \mathrm{C}(\mathrm{C} 4) ; \delta=62.4 \mathrm{ppm}, 1 \mathrm{C}(\mathrm{C} 6) ; \delta=55.1$ ppm, $1 \mathrm{C}\left(\mathrm{OCH}_{2} \mathrm{CCH}\right) ; \delta=20.9 \mathrm{ppm}, 1 \mathrm{C}\left(\mathrm{OCOCH}_{3}\right) ; \delta=20.8 \mathrm{ppm}, 2 \mathrm{C}$ (three times $\left.\mathrm{OCOCH}_{3}\right)$.

$\mathrm{R}_{\mathrm{f}}=0.38$ with toluene:ethyl acetate (3:1).

ESI-MS for $\mathrm{C}_{17} \mathrm{H}_{22} \mathrm{O}_{10}: \mathrm{m} \cdot \mathrm{z}^{-1}\left(\mathrm{M}+\mathrm{Na}^{+}\right)_{\text {calc }}=409.1 ; \mathrm{m} \cdot \mathrm{z}^{-1}\left(\mathrm{M}+\mathrm{Na}^{+}\right)_{\text {obs }}=409.2 ; \mathrm{m} \cdot \mathrm{z}^{-1}\left(\mathrm{M}+\mathrm{NH}_{4}{ }^{+}\right)_{\text {calc }}=404.2$; $\mathrm{m} \cdot \mathrm{z}^{-1}\left(\mathrm{M}+\mathrm{NH}_{4}{ }^{+}\right)_{\mathrm{obs}}=404.2$. 

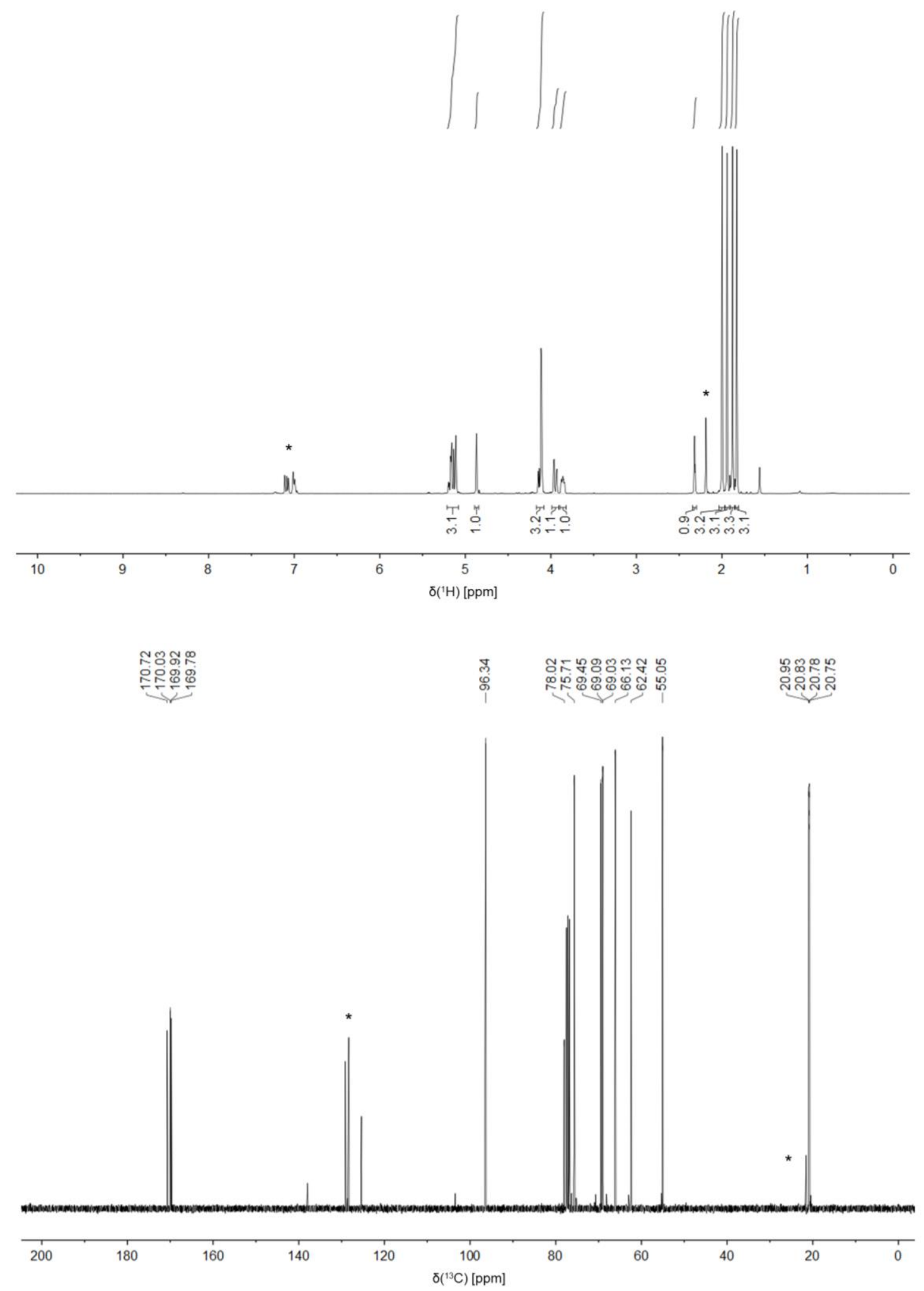

699

700 *Sample contained residual toluene. The yield was determined after removal of the solvent in vacuo. 
703

704

705

706

707

708

709

710

711

712

713

714

715

716

717

718

719

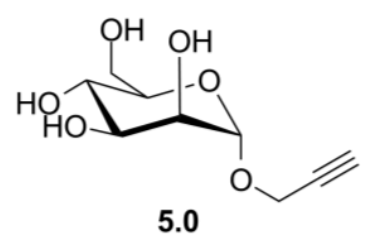

$7(188 \mathrm{mg}, 487 \mu \mathrm{mol})$ was dissolved in $\mathrm{EtOH}$ containing $33 \%$ methylamine $(13 \mathrm{ml})$ at room temperature. The mixture was stirred overnight and progress of the reaction was monitored by analytical TLC (20\% MeOH in DCM). Solvents were evaporated in vacuo and the residue was purified via column chromatography (gradient: hexane, hexane:DCM (1:1), DCM, 1\% MeOH in DCM, $5 \% \mathrm{MeOH}$ in DCM and elution with $20 \% \mathrm{MeOH}$ in DCM). Silica gel particles were removed by filtration in $\mathrm{MeOH}$ with a cellulose acetate membrane at a pore size $0.2 \mu \mathrm{m}$ to yield $\mathbf{5 . 0}(91 \mathrm{mg}$, $417 \mu \mathrm{mol}, 86 \%)$ as a white solid.

${ }^{1} \mathrm{H}$ NMR (400.0 MHz, MeOD): $\delta=4.95$ ppm, d, $1 \mathrm{H}, \mathrm{J}=1.2 \mathrm{~Hz}(\mathrm{H} 1) ; \delta=4.26$ ppm, d, $2 \mathrm{H}, \mathrm{J}=2.6$ $\mathrm{Hz}\left(\mathrm{OCH}_{2} \mathrm{CCH}\right) ; \delta=3.79$ ppm, dd, $1 \mathrm{H}, \mathrm{J}=2.2,11.7 \mathrm{~Hz}(\mathrm{H6a}) ; \delta=3.76 \mathrm{ppm}, \mathrm{dd}, 1 \mathrm{H}, \mathrm{J}=1.6,3.1 \mathrm{~Hz}$ $(\mathrm{H} 2) ; \delta=3.70 \mathrm{ppm}, \mathrm{m}, 1 \mathrm{H}(\mathrm{H} 6 \mathrm{~b}) ; \delta=3.65 \mathrm{ppm}, \mathrm{m}, 1 \mathrm{H}(\mathrm{H} 3) ; \delta=3.61 \mathrm{ppm}, \mathrm{m}, 1 \mathrm{H}(\mathrm{H} 4) ; \delta=3.50$ ppm, ddd, $1 \mathrm{H}, \mathrm{J}=2.0,5.8,9.6 \mathrm{~Hz}(\mathrm{H} 5) ; \delta=2.85 \mathrm{ppm}, \mathrm{t}, 1 \mathrm{H}, \mathrm{J}=2.4 \mathrm{~Hz}\left(\mathrm{OCH}_{2} \mathrm{CC} H\right)$.

${ }^{13} \mathrm{C}$ NMR (100.6 MHz, MeOD): $\delta=99.8 \mathrm{ppm}, 1 \mathrm{C}(\mathrm{C} 1) ; \delta=80.0 \mathrm{ppm}, 1 \mathrm{C}\left(\mathrm{OCH}_{2} \mathrm{CCH}\right) ; \delta=76.0$ ppm, $1 \mathrm{C}\left(\mathrm{OCH}_{2} \mathrm{CCH}\right) ; \delta=75.1 \mathrm{ppm}, 1 \mathrm{C}(\mathrm{C} 5) ; \delta=72.5 \mathrm{ppm}, 1 \mathrm{C}(\mathrm{C} 3) ; \delta=72.0 \mathrm{ppm}, 1 \mathrm{C}(\mathrm{C} 2) ; \delta=$ $68.5 \mathrm{ppm}, 1 \mathrm{C}(\mathrm{C} 4) ; \delta=62.8 \mathrm{ppm}, 1 \mathrm{C}(\mathrm{C} 6) ; \delta=54.8 \mathrm{ppm}, 1 \mathrm{C}\left(\mathrm{OCH}_{2} \mathrm{CCH}\right)$.

$\mathrm{R}_{\mathrm{f}}=0.36$ with $20 \% \mathrm{MeOH}$ in DCM.

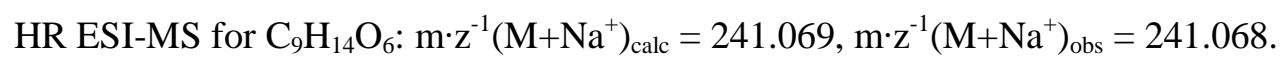




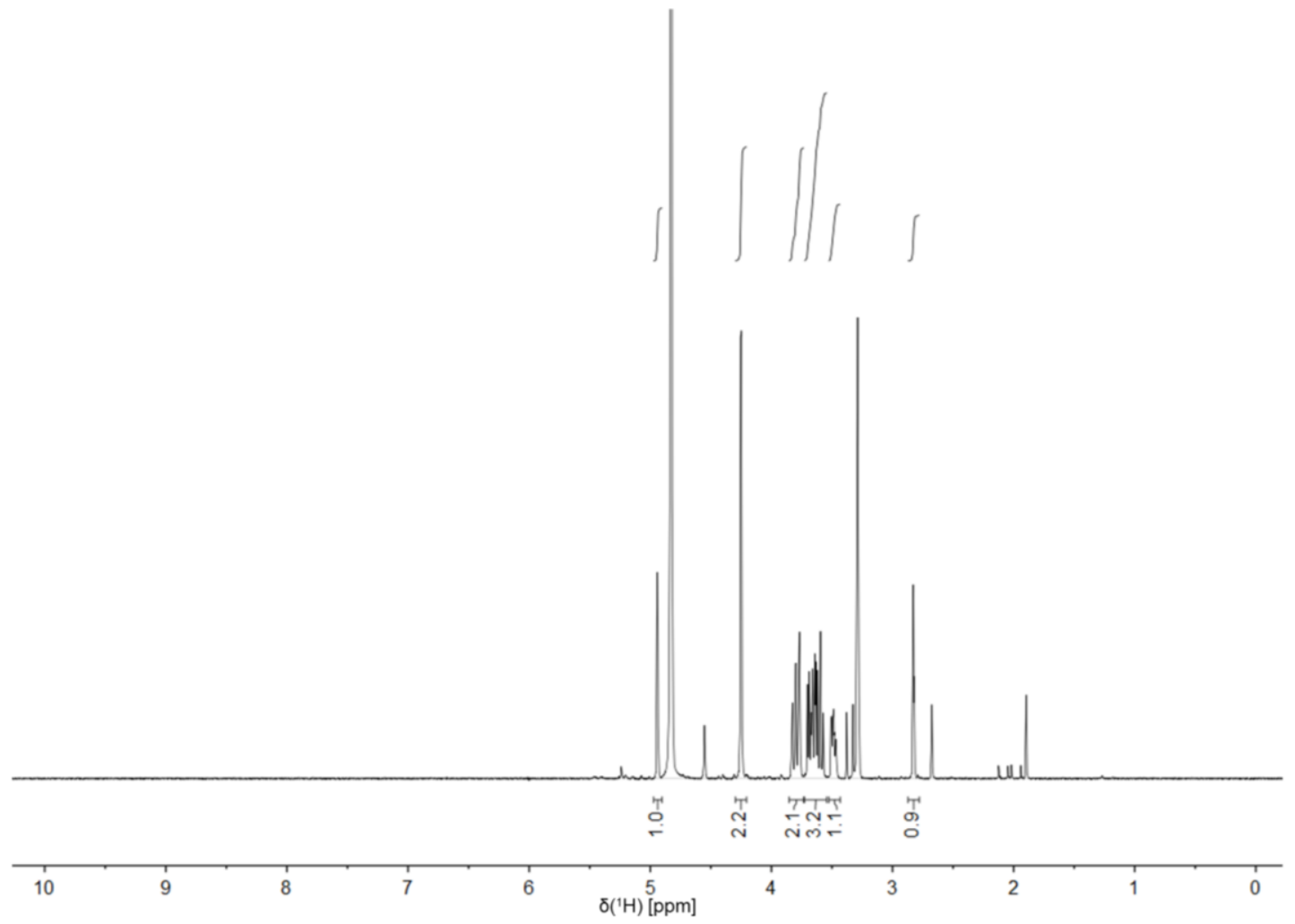

720

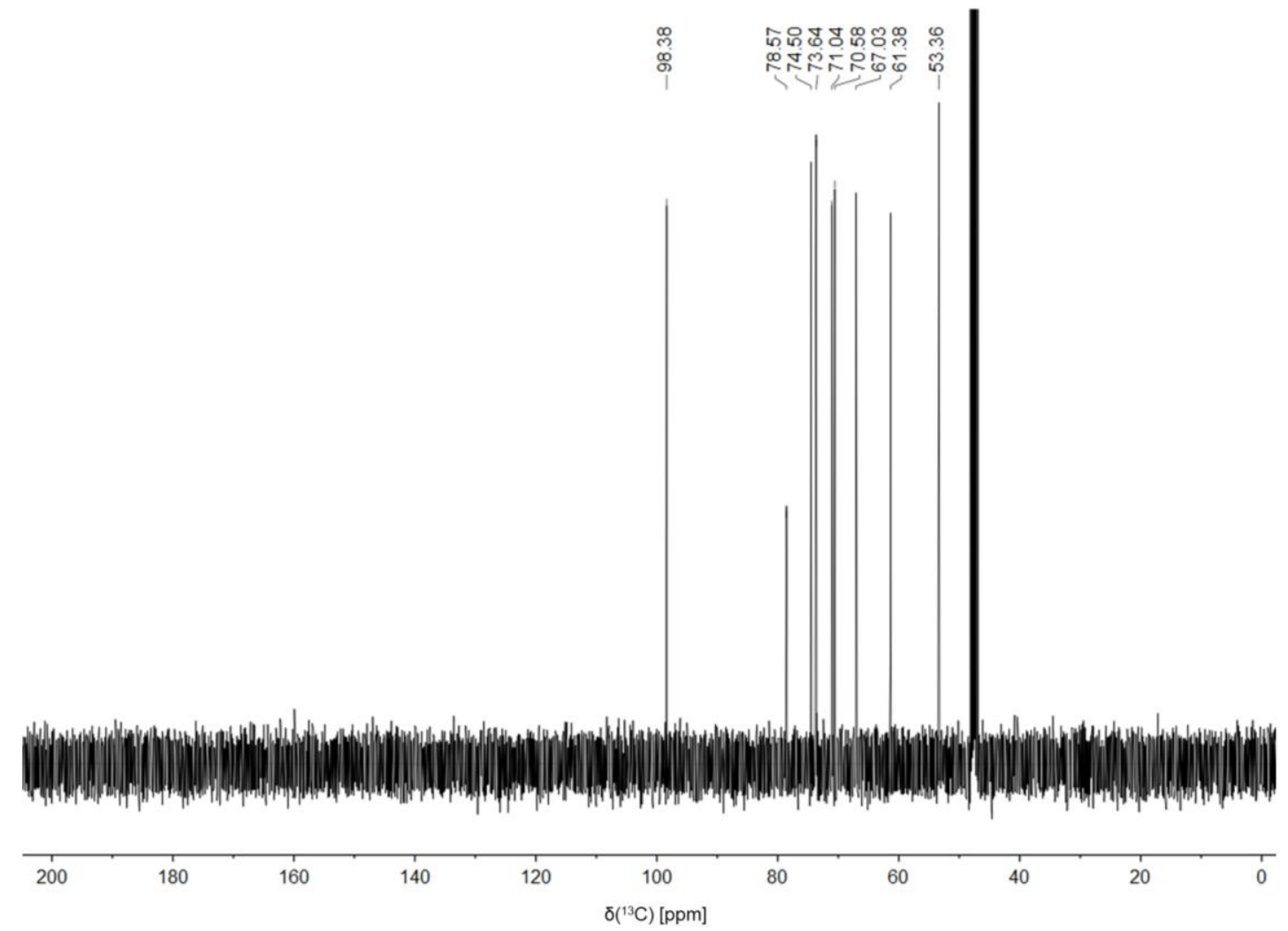




\section{${ }^{19} \mathrm{~F} \mathbf{R}_{\mathbf{2}}$-filtered NMR}

${ }^{19} \mathrm{~F} \mathrm{R}_{2}$-filtered NMR experiments were conducted on a OneNMR $600 \mathrm{MHz}$ spectrometer (Agilent). Spectra were processed in MestReNova and data analysis was performed with OriginPro $(19,22)$. Experiments utilizing the Langerin ECD were performed at a receptor concentration of $50 \mu \mathrm{M}$ in 25 $\mathrm{mM}$ Tris with $10 \% \mathrm{D}_{2} \mathrm{O}, 150 \mathrm{mM} \mathrm{NaCl}$ and $5 \mathrm{mM} \mathrm{CaCl}_{2}$ at $\mathrm{pH} 7.8$ and $25^{\circ} \mathrm{C}$. Experiments utilizing the Langerin CRD were performed at a receptor concentration of $50 \mu \mathrm{M}$ in $25 \mathrm{mM}$ HEPES with $10 \%$ $\mathrm{D}_{2} \mathrm{O}, 150 \mathrm{mM} \mathrm{NaCl}$ and $5 \mathrm{mM} \mathrm{CaCl}_{2}$ at $\mathrm{pH} 7.0$ and $25^{\circ} \mathrm{C}$. Trifluoroacetic acid served as an internal reference at a concentration of 50 or $100 \mu \mathrm{M}$. For each spectrum 128 scans were recorded in $3 \mathrm{~mm}$ sample tubes at sample volumes of $150 \mu \mathrm{l}$. Relaxation rates $\mathrm{R}_{2, \text { obs }}$ were determined with the CPMG pulse sequence by fitting Equation 1 to integrals of the ${ }^{19} \mathrm{~F}$ resonance of $\mathbf{5 . 1}$ (23). T represents the relaxation time and $I_{0}$ is the integral at a $T$ value of $0 \mathrm{~s}$. The relaxation delay $d_{1}$ was set to $2.0 \mathrm{~s}$, the acquisition time $\mathrm{t}_{\mathrm{acq}}$ was set to $0.8 \mathrm{~s}$ and the frequency of $180^{\circ}$ pulses $v_{\mathrm{CPMG}}$ was set to $500 \mathrm{~Hz}$.

$$
I=I_{0} e^{-R_{2, o b s} T}
$$

\section{Equation 1}

The $K_{D}$ and the $R_{2, b}$ value of the reporter molecule $\mathbf{5 . 1}$ were derived from Equation 2 by detection of ${ }^{19} \mathrm{~F}$ NMR relaxation rates $\mathrm{R}_{2, \text { obs }}$ in a two parameter fit $(24,25)$. $\mathrm{R}_{2, \mathrm{~b}}$ represents the relaxation rate in bound state of the ligand and $\mathrm{p}_{\mathrm{b}}$ is the bound fraction of the ligand while $[\mathrm{L}]_{\mathrm{T}}$ and $[\mathrm{P}]_{\mathrm{T}}$ represent the concentrations of ligand and receptor, respectively. The relaxation rate of the free ligand $\mathrm{R}_{2, \mathrm{f}}$ was measured at $0.1 \mathrm{mM} \mathrm{5.1}$ in absence of the receptor. The EDTA control experiment was conducted at $12.5 \mathrm{mM}$ 5.1. To ensure the validity of Equation 2, the chemical exchange contribution $\mathrm{R}_{2, \mathrm{ex}}$ was estimated by ${ }^{19} \mathrm{~F}$ NMR relaxation dispersion experiments at $0.1 \mathrm{mM} \mathbf{5 . 1}$.

$$
R_{2, o b s}=R_{2, f}+\left(R_{2, b}-R_{2, f}\right) p_{b}
$$

with

$$
p_{b}=\left(\frac{[P]_{T}+[L]_{T}+K_{D}-\sqrt{\left([P]_{T}+[L]_{T}+K_{D}\right)^{2}-4[P]_{T}[L]_{T}}}{2[L]_{T}}\right)
$$

\section{Equation 2}

For the competitive binding experiments in Setup 1, binding of $0.1 \mathrm{mM} \mathbf{5 . 1}$ to the ECD was detected at five or more competitor concentrations $\left[\mathrm{I}_{\mathrm{T}}\right.$. Equation 3 served to derive $[\mathrm{P}]_{\mathrm{T}}$ and $\mathrm{K}_{\mathrm{I}}$ values from $\mathrm{R}_{2, \text { obs }}$ values in a two parameter fit (25). The $\mathrm{pH}$ value of stock solutions of $\mathbf{5 . 1 1}$ was adjusted to 7.8 prior to titration experiments using $1 \mathrm{M} \mathrm{NaOH}$. In Setup $2,[\mathrm{P}]_{\mathrm{T}}$ values were directly calculated from 
the data point at $0.1 \mathrm{mM} \mathbf{5 . 1}$ and in absence of competitor via Equation 1. Subsequently, $\mathrm{K}_{\mathrm{I}}$ values were estimated from Equation 2 in a one parameter fit. Data points were selected for the evaluation of the assay performance if the competitor concentrations fell within one order of magnitude of the $\mathrm{K}_{\mathrm{I}}$ value determined in Setup 1. Deviations from Setup 1 were quantified via the calculation of $\Delta \mathrm{K}_{\mathrm{I}}$ values.

$$
R_{2, o b s}=R_{2, f}+\left(R_{2, b}-R_{2, f}\right) p_{b}
$$

$$
p_{b}=\frac{2 \cos \left(\frac{\theta}{3}\right) \sqrt{a^{2}-3 b}-a}{3 K_{D}+2 \cos \left(\frac{\theta}{3}\right) \sqrt{a^{2}-3 b}-a}
$$

$$
\begin{gathered}
\theta=\cos ^{-1}\left(\frac{-2 a^{3}+9 a b-27 c}{2 \sqrt{\left(a^{2}-3 b\right)^{3}}}\right), \quad a=K_{D}+K_{I}+[L]_{T}+[I]_{T}-[P]_{T} \\
b=\left([I]_{T}-[P]_{T}\right) K_{D}+\left([L]_{T}-[P]_{T}\right) K_{D}+K_{I} K_{D}, \quad \mathrm{c}=-K_{I} K_{D}[P]_{T}
\end{gathered}
$$

\section{Equation 3}

758 The fragment screening was conducted using the ECD in presence of $10 \%$ DMSO and $0.01 \%$ Tween759 20. The influence of the additives on assay performance was evaluated via titration and screening experiments with Man. Additionally, the mean $\mu_{\text {Reference }}$ and standard deviation $\sigma$ of $R_{2}$,obs values in absence of competitor were estimated from independent experiments $(n=9)$. Overall, 290 fragments (Key Organics) were randomly selected from our in-house library. These fragments were binned into mixtures of 5 or 6 and screened at concentration of $0.5 \mathrm{mM}$. A $3 \sigma$-threshold was utilized to define screening hits and estimated $\mathrm{K}_{\mathrm{I}}$ values for Man and $\mathbf{8}$ were determined in Setup 2. 


\section{${ }^{15}$ N HSQC NMR}

${ }^{15} \mathrm{~N}$ HSQC NMR experiments were conducted on a OneNMR $600 \mathrm{MHz}$ spectrometer (Agilent). Spectra were processed in NMRPipe (26). Data analysis was performed in CCPN Analysis and OriginPro $(22,27)$. Experiments were conducted with Langerin CRD concentrations between 160 and $200 \mu \mathrm{M}$ in $25 \mathrm{mM}$ HEPES with $10 \% \mathrm{D}_{2} \mathrm{O}, 150 \mathrm{mM} \mathrm{NaCl}$ and $5 \mathrm{mM} \mathrm{CaCl}_{2}$ at $\mathrm{pH} 7.0$ and $25^{\circ} \mathrm{C}$. DSS served as an internal reference at a concentration of $100 \mu \mathrm{M}$. Spectra were acquired with 128 increments and 8 scans per increment for $500 \mu \mathrm{l}$ samples in $5 \mathrm{~mm}$ sample tubes and 32 scans per increments for $150 \mu \mathrm{l}$ samples in $3 \mathrm{~mm}$ sample tubes. The relaxation delay $\mathrm{d}_{1}$ was set to $0.15 \mathrm{~s}$ and the acquisition time $\mathrm{t}_{\mathrm{acq}}$ was set to $1.35 \mathrm{~s}$. The W5 Watergate pulse sequence was utilized for solvent suppression (28). CSPs for receptor resonances in the fast or fast-to-intermediate exchange regime observed upon titration with ligand were calculated via Equation 4 (29).

$$
C S P=\sqrt{\frac{\delta\left({ }^{1} \mathrm{H}\right)^{2}+(0.15 \delta(15 N))^{2}}{2}}
$$

\section{Equation 4}

Resonances that displayed CSP values higher than $0.04 \mathrm{ppm}$ at the highest ligand concentration were selected for the determination of $\mathrm{K}_{\mathrm{D}}$ values via Equation 5 in a global two parameter fit (29). $\mathrm{CSP}_{\max }$ represents the CSP value observed upon saturation of the binding site.

$$
C S P=C S P_{\text {max }} p_{b}
$$

with

$$
p_{b}=\left(\frac{[P]_{T}+[L]_{T}+K_{D}-\sqrt{\left([P]_{T}+[L]_{T}+K_{D}\right)^{2}-4[P]_{T}[L]_{T}}}{2[P]_{T}}\right)
$$

\section{Equation 5}

The $\mathrm{pH}$ value of stock solutions of $\mathbf{5 . 1 1}$ was adjusted to 7.0 prior to titration experiments using $1 \mathrm{M}$ $\mathrm{NaOH}$. 


\section{${ }^{1}$ H STD NMR}

$787{ }^{1} \mathrm{H}$ STD NMR experiments were conducted on a OneNMR $600 \mathrm{MHz}$ spectrometer (Agilent) and 788 spectra were processed in MestReNova $(19,30)$. Experiments were conducted utilizing the Langerin 789 ECD at a receptor concentration of $25 \mu \mathrm{M}$ in $25 \mathrm{mM}$ Tris- $\mathrm{d}_{11}$ with $100 \% \mathrm{D}_{2} \mathrm{O}, 150 \mathrm{mM} \mathrm{NaCl}$ and 5 $790 \mathrm{mM} \mathrm{CaCl}_{2}$ at $\mathrm{pH} 7.8$ and $25^{\circ} \mathrm{C}$. The experiment was repeated in absence of receptor to exclude STD 791 effects due to direct saturation of fragments. Residual $\mathrm{H}_{2} \mathrm{O}$ served as an internal reference. For each 792 spectrum 512 scans were recorded in $5 \mathrm{~mm}$ sample tubes at sample volumes of $500 \mu$ l. Saturation was 793 implemented via a train of Gauss pulses at a saturation time $t_{\text {sat }}$ of $4.0 \mathrm{~s}$. The on-resonance irradiation 794 frequency $v_{\text {sat }}$ was set to $0.0 \mathrm{ppm}$ and the off-resonance irradiation frequency $v_{\text {ref }}$ was set to $80.0 \mathrm{ppm}$. 795 The relaxation delay $\mathrm{d}_{1}$ was set to $0.0 \mathrm{~s}$ and the acquisition time $\mathrm{t}_{\text {acq }}$ was set to $2.0 \mathrm{~s}$. The DPFGSE 796 pulse sequence was utilized for solvent suppression (31). Receptor resonances were suppressed via a $797 \mathrm{~T}_{1, \text { rho }}$ filter at a relaxation time $\tau$ of $35 \mathrm{~ms}$. Resonances of the analyzed fragment mixture were assigned 798 by comparison to previously acquired ${ }^{1} \mathrm{H}$ NMR spectra of the individual fragments. 
801 1. Duus, J., Gotfredsen, C. H., and Bock, K. (2000) Carbohydrate structural determination by NMR spectroscopy: modern methods and limitations. Chem. Rev. 100, 4589-4614.

2. Feinberg, H., Taylor, M. E., Razi, N., McBride, R., Knirel, Y. A., Graham, S. A., Drickamer, K., and Weis, W. I. (2011) Structural basis for langerin recognition of diverse pathogen and

6. Chemical Computing Group (2013) Molecular Operating Environment, 2013.08. mammalian glycans through a single binding site. J. Mol. Biol. 405, 1027-1039.

3. Mannhold, R., Poda, G. I., Ostermann, C., and Tetko, I. V. (2009) Calculation of molecular lipophilicity: State-of-the-art and comparison of $\log \mathrm{P}$ methods on more than 96,000 compounds. J. Pharm. Sci. 98, 861-893.

4. Chen, I. J., and Hubbard, R. E. (2009) Lessons for fragment library design: analysis of output

5. Keseru, G. M., Erlanson, D. A., Ferenczy, G. G., Hann, M. M., Murray, C. W., and Pickett, S. D. (2016) Design Principles for Fragment Libraries: Maximizing the Value of Learnings from Pharma Fragment-Based Drug Discovery (FBDD) Programs for Use in Academia. J. Med. Chem. Online.

Case, D. A., Darden, T. A., Cheatham, T. E., Simmerling, C. L., Wang, J., Duke, R. E., Luo, R., Crowley, M., R.C.Walker, Zhang, W., Merz, K. M., B.Wang, Hayik, S., Roitberg, A., Seabra, G., Kolossváry, I., K.F.Wong, Paesani, F., Vanicek, J., X.Wu, Brozell, S. R., Steinbrecher, T., Gohlke, H., Yang, L., Tan, C., Mongan, J., Hornak, V., Cui, G., Mathews, D. H., Seetin, M. G., Sagui, C., Babin, V., and P.A. Kollman (2008) AMBER, 10.

Gerber, P. R., and Müller, K. (1995) MAB, a generally applicable molecular force field for structure modelling in medicinal chemistry. J. Comput. Aided Mol. Des. 9, 251-268.

9. Halgren, T. A. (1996) Merck molecular force field. I. Basis, form, scope, parameterization, and performance of MMFF94. J. Comput. Chem. 17, 490-519.

10. Berthold, M. R., Cebron, N., Dill, F., Gabriel, T. R., Koetter, T., Meinl, T., Ohl, P., Sieb, C., Thiel, K., and Wiswedel, B. (2007) KNIME: The Konstanz Information Miner, in Studies in Classification, Data Analysis, and Knowledge Organization, Springer.

11. Feinberg, H., Rowntree, T. J., Tan, S. L., Drickamer, K., Weis, W. I., and Taylor, M. E. (2013) Common polymorphisms in human langerin change specificity for glycan ligands. $J$. Biol. Chem. 288, 36762-36771.

12. Chen, V. B., Arendall, W. B., 3rd, Headd, J. J., Keedy, D. A., Immormino, R. M., Kapral, G. J., Murray, L. W., Richardson, J. S., and Richardson, D. C. (2010) MolProbity: all-atom structure validation for macromolecular crystallography. Acta Crystallogr. Sect. D-Biol. Crystallogr. 66, 12-21.

13. Labute, P. (2010) LowModeMD - implicit low-mode velocity filtering applied to conformational search of macrocycles and protein loops. J. Chem. Inf. Model. 50, 792-800.

14. Oprea, T. I. (2000) Property distribution of drug-related chemical databases. J. Comput. Aided Mo.l Des. 14, 251-264. 
16. Stambach, N. S., and Taylor, M. E. (2003) Characterization of carbohydrate recognition by langerin, a C-type lectin of Langerhans cells. Glycobiology 13, 401-410.

17. Gasteiger, E., Hoogland, C., Gattiker, A., Duvaud, S., Wilkins, M. R., Appel, R. D., and Bairoch, A. (2005) Protein identification and analysis tools on the ExPASy server, in The Proteomics Protocols Handbook (Walker, J. M., Ed.), 571-607, Humana Press.

18. Duus, J., Gotfredsen, C. H., and Bock, K. (2000) Carbohydrate structural determination by

19. Mestrelab Research (2015) MestreNova, 10.0.2.

20. Veselý, J., Ledvina, M., Jindrich, J., Šaman, D., and Trnka, T. (2003) Improved synthesis of 1,2-trans-acetates and 1,2-trans ethyl 1-thioglycosides derived from 3,4,6-tri-O-acetyl-2deoxy-2-phthalimido-d-hexopyranosides. Collect. Czech. Chem. Comm. 68, 1264-1274.

21. Hasegawa, T., Numata, M., Okumura, S., Kimura, T., Sakurai, K., and Shinkai, S. (2007) Carbohydrate-appended curdlans as a new family of glycoclusters with binding properties both for a polynucleotide and lectins. Org. Biomol. Chem. 5, 2404-2412.

22. OriginLab (2015) OriginPro, 9.1.

23. Carr H. Y., and M., P. E. (1954) Effects of diffusion on free precession in nuclear magnetic resonance experiments. Phys. Rev. 94.

24. Dalvit, C. (2007) Ligand- and substrate-based 19F NMR screening: Principles and applications to drug discovery. Prog. Nucl. Mag. Res. Sp. 51, 243-271.

25. Roehrl, M. H., Wang, J. Y., and Wagner, G. (2004) A general framework for development and data analysis of competitive high-throughput screens for small-molecule inhibitors of proteinprotein interactions by fluorescence polarization. Biochemistry 43, 16056-16066.

26. Delaglio, F., Grzesiek, S., Vuister, G. W., Zhu, G., Pfeifer, J., and Bax, A. (1995) NMRPipe: A multidimensional spectral processing system based on UNIX pipes. J. Biomol. NMR 6, 277293.

27. Vranken, W. F., Boucher, W., Stevens, T. J., Fogh, R. H., Pajon, A., Llinas, M., Ulrich, E. L., Markley, J. L., Ionides, J., and Laue, E. D. (2005) The CCPN data model for NMR spectroscopy: development of a software pipeline. Proteins 59, 687-696.

28. Liu, M., Mao, X.-a., Ye, C., Huang, H., Nicholson, J. K., and Lindon, J. C. (1998) Improved WATERGATE pulse sequences for solvent suppression in NMR spectroscopy. J. Magn. Reson. 132, 125-129.

29. Williamson, M. P. (2013) Using chemical shift perturbation to characterise ligand binding. Prog. Nucl. Magn. Reson. Spectrosc. 73, 1-16.

30. Mayer, M., and Meyer, B. (1999) Characterization of ligand binding by saturation transfer difference NMR spectroscopy. Angew. Chem. Int. Ed. 38, 1784-1788.

31. Hwang, T. L., and Shaka, A. J. (1995) Water Suppression That Works - Excitation Sculpting Using Arbitrary Wave-Forms and Pulsed-Field Gradients. J. Magn. Reson. Ser. A 112, 275279. 